\title{
THE SEMANTICS OF VALUE-RANGE NAMES AND FREGE'S PROOF OF REFERENTIALITY
}

\author{
MATTHIAS SCHIRN \\ Munich Center for Mathematical Philosophy, University of Munich
}

\begin{abstract}
"A word without a determinate meaning has no meaning for mathematics” (Frege, 'Über die Grundlagen der Geometrie' II, 1906, Frege, 1967, p. 290).
\end{abstract}

\begin{abstract}
In this article, I try to shed some new light on Grundgesetze §10, §29-\$31 with special emphasis on Frege's criteria and proof of referentiality and his treatment of the semantics of canonical value-range names. I begin by arguing against the claim, recently defended by several Frege scholars, that the first-order domain in Grundgesetze is restricted to value-ranges (including the truth-values), but conclude that there is an irresolvable tension in Frege's view. The tension has a direct impact on the semantics of the concept-script, not least on the semantics of value-range names. I further argue that despite first appearances truth-value names (sentences) play a distinguished role as semantic "target names" for "test names" in the criteria of referentiality (\$29) and do not figure themselves as "test names" regarding referentiality. Accordingly, I show in detail that Frege's attempt to demonstrate that by virtue of his stipulations "regular" value-range names have indeed been endowed with a unique reference, can plausibly be regarded as a direct application of the context principle. In a subsequent section, I turn to some special issues involved in $\$ 10$. $\S 10$ is closely intertwined with $\S 31$ and in my and Richard Heck's view would have been better positioned between $\S 30$ and $\S 31$. In a first step, I discuss the piecemeal strategy which Frege applies when he attempts to bestow a unique reference on value-range names in $\S 3, \S 10-\S 12$. In a second step, I critically analyze his tentative, but predictably unsuccessful proposal (in a long footnote to $§ 10$ ) to identify all objects whatsoever, including those already clad in the garb of value-ranges, with their unit classes. In conclusion, I present two arguments for my claim that Frege's identification of the True and the False with their unit classes in $\S 10$ is illicit even if both the permutation argument and the identifiability thesis that he states in $\$ 10$ are regarded as formally sound. The first argument is set out from the point of view of the syntax of his formal language. It suggests though that a reorganization of the exposition of the concept-script would have solved at least one of the problems to which the twin stipulations in $\S 10$ give rise. The second argument rests on semantic considerations. If it is sound, it may call into question, if not undermine the legitimacy of the twin stipulations.
\end{abstract}

\$1. Introduction. When Frege had completed his philosophical masterpiece Die Grundlagen der Arithmetik (1884), he knew that he could not rest on his laurels. In Grundlagen, he had only outlined the logicist programme, but it was underpinned with much philosophical argument. After Grundlagen, the requisite formal and complete elaboration of the programme was a tall order. The most pressing task was to introduce in a

Received: February 18, 2016.

2010 Mathematics Subject Classification: 03-03.

Key words and phrases: all-encompassing domain, criteria of referentiality, context principle, identifiability thesis, piecemeal strategy, referential indeterminacy, regular value-range names, unit classes. 
methodologically sound fashion logical objects of a fundamental and irreducible kind with which all numbers could justifiably be identified. The introduction of those objects was intended to proceed via logical abstraction, namely by stating purely logical criteria of identity for them and in this way to fix the reference of a suitable term-forming operator.

Needless to say, Frege's introduction of value-ranges of (first-level) functions and valuerange names in his opus magnum Grundgesetze der Arithmetik (vol. I 1893, vol. II 1903) via logical abstraction did not emerge from out of the blue in his work. It had its roots in his earlier attempt in Grundlagen to define cardinal numbers paradigmatically as logical objects and, moreover, in his envisaged definitional introduction of the real and complex numbers. The latter was designed to proceed along the lines of the introduction of the cardinals, ${ }^{1}$ namely by starting with a tentative contextual definition of a suitable number operator in terms of an abstraction principle whose right-hand side-a second-order or higher-order equivalence relation qua criterion of identity-was couched in purely logical vocabulary, and by finally defining these numbers explicitly as extensions of concepts or more specifically: as equivalence classes of the relevant equivalence relation. ${ }^{2}$

In Frege's view, the need to replace the tentative contextual definitions with explicit definitions was only due to the emergence of the Julius Caesar problem, or more accurately, to the emergence of a whole family of Caesar problems for cardinal, real, and complex numbers. Yet in order to justify the explicit definitions, extensions of concepts had to be introduced as logical objects in a methodologically correct manner instead of assuming familiarity with them and taking their logical nature tacitly for granted, as Frege had done in Grundlagen. In my opinion, it was precisely these ungrounded assumptions and the related failure of his attempt to resolve the Julius Caesar problem for cardinal numbers by defining them as extensions of concepts that overshadowed or even undermined the logicist project as sketched in Grundlagen.

Unsurprisingly, there are not only striking parallels, but also remarkable differences between the old and the new strategy of fixing the mechanisms for identifying reference to logical objects. In this essay, following the Introduction, I shall critically discuss the new strategy, in particular Frege's method of endowing canonical value-range names (allegedly) with a unique reference as well as his attempt to demonstrate that regular value-range names $^{3}$ are in fact referential. He probably thought that in Grundgesetze, $\$ 10-\S 12$ he had succeeded in getting rid of the indeterminacy of the references of value-range names, arising from a semantic stipulation in $\S 3$, by specifying the values of the primitive firstlevel functions for the truth-values and value-ranges as arguments. The stipulation in $\S 3$ is framed in informal, metalinguistic vocabulary and reads as follows:

I use the words 'the function $\Phi(\xi)$ has the same value-range as the function $\Psi(\xi)$ ' generally as coreferential [gleichbedeutend] with the words 'the functions $\Phi(\xi)$ and $\Psi(\xi)$ always have the same value for the same argument'.

Henceforth I call this stipulation the contextual stipulation because it is immediately reminiscent of the attempted contextual definition of the direction operator in Grundlagen, $\S 65$, which in fact reads very similarly. The contextual stipulation, which later in Grundgesetze

1 See Schirn (2003, 2006b, 2014b, 2016, 2018a,b).

2 Regarding Frege's foundation of real analysis in Grundgesetze see Schirn (2013, 2014a). Overshadowed by Russell's Paradox, Frege's theory of real numbers remained a fragment.

3 In Frege (1893), §31, Frege calls value-range names that are formed from referential monadic first-level function-names regular value-range names (rechte Wertverlaufsnamen). 
appears enshrined in the formal version of Axiom $\mathrm{V}: \vdash(\dot{\varepsilon} f(\varepsilon)=\dot{\alpha} g(\alpha))=(\mathfrak{a}-f(\mathfrak{a})=$ $g(\mathfrak{a}))$ (cf. Frege (1893), §9, §20), is nonstandard because, unlike the elucidations of the other primitive function-names of Frege's system, it does not directly assign a reference (and a sense) to the name of the value-range function by stating the values that this function receives for appropriate arguments, in this case for monadic first-level functions as arguments. It is rather designed to fix at least partially the reference of the name of that function by licensing the mutual transition from one mode of speaking which involves that name to another which does not (cf. Frege (1903), §146). Note that in contrast to the contextual stipulation, Basic Law V asserts something: the value-range of $f$ is identical with the value-range of $g$ if and only if $f$ and $g$ are coextensive.

After having determined the references of value-range names in a piecemeal fashionapparently to his satisfaction-Frege thought that it was nonetheless imperative to demonstrate that regular value-range names, and consequently the primitive value-range operator " $\dot{\varepsilon} \varphi(\varepsilon)$ ", have indeed been endowed with a unique reference. To be sure, this demonstration formed only a part of the proof in Grundgesetze, $\S 31$ that every well-formed name of the concept-script has a reference. As we shall see, the proof proceeds by induction on the complexity of concept-script names. Yet to demonstrate that regular value-range names refer to something required considerably more effort from Frege than the remainder of the proof in $\S 31$, which in the light of his initial assumption that truth-value names (sentences) are referential in their own right, turned out to be almost trivial. In this essay, I shall discuss some important aspects of the proof of referentiality in $\$ 31$ including its revealing prelude in $\S 29-\$ 30$, with special emphasis on what Frege regards as the critical spot of the proof: examining the semantics of value-range names.

Before Russell discovered the contradiction in the logical system of Grundgesetze, Frege considered Axiom $\mathrm{V}$ to be the fulcrum of his foundational project. He believed that it is the appropriate means of coming into epistemic contact with value-ranges once the indeterminacy of the references of canonical value-range names ${ }^{4}$ was removed, as he must have believed at the end of $\S 12$ of Grundgesetze, if we give credence to what he says in $\$ 10$ in anticipation of the stipulations to be made in $\S 11-\S 12$ regarding the definite description operator and the name of the conditional function. Since Frege construed the question "How do we grasp logical objects, in particular, the numbers?" as the fundamental problem

4 Once the second-level function-name " $\varepsilon \varphi(\varepsilon)$ " is available in the formal language and we are entitled to apply the rule of insertion as one of the two permissible modes of forming new names from the primitive function-names, we may regard any term that results from the insertion of a monadic first-level function-name into the argument-place of " $\dot{\varepsilon} \varphi(\varepsilon)$ " as a canonical valuerange name. Similarly, we may call equations in which the terms flanking " $=$ " are both canonical value-range names canonical value-range equations. Due to certain stipulations that Frege makes in $\S 10-\S 11$, a truth-value name such as " $-\mathfrak{a}-\mathfrak{a}=\mathfrak{a}$ " refers to $\dot{\varepsilon}(-\varepsilon)$, and a definite description such as "\} \grave { \varepsilon } ( - \varepsilon ) \text { " likewise refers to } \grave { \varepsilon } ( - \varepsilon ) \text { . Thus, "\} \grave { \varepsilon } ( - \varepsilon ) = \grave { \varepsilon } ( - \varepsilon ) \text { ", for example, is not } a canonical value-range equation, although the terms flanking " $=$ " on both sides refer to a valuerange, indeed to the same value-range. Note that the term "canonical" as applied to value-range names is not tantamount to Frege's term "regular". "Canonical" is meant as a purely syntactic attribute, while Frege introduces "regular" as a semantic attribute of certain canonical value-range names, namely of those that — at a certain stage in his proof of referentiality — can be formed from monadic function-names of first level already shown to be referential. Of course, if the proof of referentiality for all well-formed concept-script names in $\$ 31$ were successful (as Frege believed that it was), then every canonical value-range name would deserve the attribute "regular", since all one-place first-level function-names of the formal language would be referential. For the time being, I disregard names of double value-ranges; they can be formed from the names of simple value-ranges in a straightforward way. 
of arithmetic, he knew from the very outset of his foundational enterprise in Grundgesetze that only by conferring on every canonical value-range name a unique reference and by subsequently proving this could he justify the use of these names in his formal language and place his logicist project on a firm foundation.

The following $\S 2, \S 3$, and $\S 4$ are crucial, if not indispensable, for an adequate understanding and critical assessment of Frege's strategy in Grundgesetze, §30-\$31. Furthermore, they are of considerable interest in their own right and may foster not only our understanding of the exposition of the concept-script in general, but also of the close links that Frege saw between the formation rules and the conditions of referentiality for concept-script names in particular. In $\S 2$, I shall describe and analyze the conflict between two clashing assumptions that Frege seems to make in different contexts in Grundgesetze concerning the range of the first-order variables of his formal system. To all appearances, he takes the first-order domain to be all-inclusive when he elucidates the primitive, logically simple first-level functions and defines certain complex first-level functions of his system. By contrast, in $\S 10$, where he diagnoses the indeterminacy of the references of value-range names and offers a key to solving the problem by first setting out his permutation argument, by subsequently stating his identifiablity thesis ${ }^{5}$ and identifying accordingly the True and the False with special value-ranges (their unit classes), ${ }^{6}$ and by finally indicating further steps to be carried out, he seems to be operating under the assumption that the first-order domain embraces only those objects whose existence is required by the axioms of his system: the two truth-values and value-ranges. ${ }^{7}$ And this assumption appears to guide even more obviously his proof of referentiality for concept-script names in $\S 31$. Nonetheless, I argue against the claim, recently defended by some Frege scholars, that the domain of first-order quantification in Grundgesetze is in fact restricted to value-ranges (including the truth-values), but conclude that there is an irresolvable tension in Frege's view. Plainly, if the first-order domain is taken to be all-encompassing, then Frege's intended complete determination of the references of canonical value-range names in $\S 10-\S 12$ is inevitably bound to fail. Moreover, any subsequent proof of referentiality for all concept-script names would be an exercise in futility.

In $\S 3$, I analyze the formation rules of the concept-script (Frege, 1893, §26) and their interplay, while in $\$ 4$ I discuss Frege's criteria of referentiality (Frege, 1893, §29). Both the formation rules and the criteria of referentiality essentially underlie his revealing

5 Frege's claim in $\S 10$ : “Thus, without contradicting our equating ' $\dot{\varepsilon} \Phi(\varepsilon)=\dot{\alpha} \Psi(\alpha)$ ' with ' $\mathfrak{a}-\Phi(\mathfrak{a})=\Psi(\mathfrak{a})$ ', it is always possible to determine that an arbitrary value-range be the True and another arbitrary value-range be the False" was appropriately called the identifiability thesis by Schroeder-Heister (1987).

6 The contextual stipulation in Frege (1893), $\$ 3$ is later embodied in the formal version of Basic Law V. By contrast, the identification of the True and the False with their unit classes in $\S 10$ is not formalized, let alone laid down as an axiom of the system. It is hard to see in any case that it could have been given the status of a logical axiom, since (a) it lacks the most salient feature of logic, which is utmost generality; and (b) it is far from being self-evident (unmittelbar einleuchtend). As a matter of fact, for Frege its plausibility and formal legitimacy rest entirely on his permutation argument which runs through several stages and is hardly self-evident.

7 At the end of $\S 10$, Frege signals that he is pursuing a dual strategy to be carried out each time at one fell swoop, as follows: (a) further specifying the value-ranges and at the same time (b) determining the primitive first-level functions that must still be introduced for the purpose of laying the logical foundations of arithmetic and are not reducible to functions already known by stipulating what values the latter should have for the former as arguments. (a) and (b) are only two sides of the same coin, which is the act of stipulating what values a primitive first-level function should have for value-ranges as arguments. 
considerations in $\$ 30$ and his subsequent proof in $\S 31$. Unfortunately, the criteria of referentiality are affected by circularity. Although Frege was aware of this and apparently regarded it as unavoidable, he probably did not carefully assess the consequences that it had for his proof of referentiality. Moreover, the criteria can only guarantee that all names, which we obtain from the primitive function-names via insertion, refer to something if the primitive names have a reference. Yet the criteria cannot ensure that those names whose construction involves the application of a gap formation rule, governing the extraction of function-names from more complex names, are likewise referential, if the primitive names refer to something. On the positive side, I argue that despite first appearances sentences (truth-value names) play a distinguished role as semantic "target names" for "test names" in the criteria of referentiality and do not themselves figure as "test names" regarding referentiality. This role is quite in the spirit of the context principle known from Grundlagen, which I basically endorse regarding the semantics of both natural and formal languages. In §7, I show in detail that Frege's attempt to demonstrate that by virtue of his stipulations regular value-range names have indeed been endowed with a unique reference, can plausibly be regarded as a direct application of the context principle, construed as a principle concerning the references of subsentential expressions.

To conclude these introductory remarks, let me emphasize that the strategy which Frege pursues in Grundgesetze, $\S 3, \S 10-\S 12$ on the one hand and the strategy which he applies in $\S 30-\$ 31$ on the other are closely intertwined, but do not coincide, nor are their objectives exactly the same. In $\S 31$, he relies heavily on his previous stipulations, in particular on his identification of the two truth-values with their unit classes in $\$ 10$ which, as I said, is taken to be the key to resolving the indeterminacy of the references of canonical value-range terms. Yet unlike the procedure in $\S 3, \S 10-\S 12$, the proof in $\S 31$ is not a matter of making any stipulations. The relevant stipulations for the purpose of fixing the references of the primitive function-names were already made at an earlier stage. Casually speaking, the task in $\S 31$ is to demonstrate that the previous stipulations do their job. If they do, then, Frege thinks, we can be sure that every primitive name has a unique reference. And the mechanics of the formation rules is supposed to guarantee that all well-formed nonprimitive names of the concept-script, including the canonical value-range names, inherit the property of being referential from the primitive names. Towards the end of my essay, I return to Grundgesetze, §10. In $\S 8.1$, I analyze the piecemeal strategy which Frege proposes and partly carries out in $\S 10$. The objective of this strategy is to resolve the indeterminacy that he characterizes at the outset of $\S 10$ or in other words: to fix the references of value-range names uniquely. In $§ 8.2$, I critically assess Frege's line of thought in the long footnote to $\S 10$. Frege explores here the possibility of generalizing the twofold stipulation in $\S 10$. In the concluding $§ 9$, I present two arguments for my claim that his identification of the True and the False with their unit classes in $\S 10$ is illicit even if both the permutation argument and the identifiability thesis that he states in $\S 10$ are regarded as formally sound. The first argument is set out from the point of view of the syntax of Frege's formal language. It suggests though that a rearrangement of the exposition of the concept-script would have solved at least one of the problems to which the twin stipulations in $\S 10$ give rise. The second argument rests on semantic considerations. If it carries conviction, it may call into question, if not undermine the rightfulness of the twin stipulations.

\$2. The range of the first-order variables in Grundgesetze. Several Frege scholars, including Dummett (1991), Heck (1997, 1999, 2005, 2011, 2012), Ruffino (2002), and Blanchette (2012a, 2012b, 2015, 2016) assume or even categorically contend that in 
Grundgesetze Frege takes the first-order domain to comprise only the truth-values and value-ranges-henceforth referred to as assumption $A$. We already know that the way Frege proceeds in $\S 10$ and especially in $\S 31$ seems to favour $A$. Moreover, his remark in $\S 9$ that by introducing his notation for value-ranges we also extend the domain of what can appear as the argument of a (first-level) function, might be taken to suggest that it is the expressive power or the referential repertoire of the concept-script that determines the range of admissible arguments of a first-level function. Thus, it could seem that in Frege's view it is sufficient to specify the values of $\xi=\zeta$ - and of every primitive firstlevel function that is introduced at a later stage and which is not completely reducible to a primitive first-level function already known - for value-ranges and the truth-values as arguments in order to resolve the indeterminacy of the reference of the value-range operator because the concept-script does not and need not contain any means of referring to other objects. Again, it is true that the existence of any objects other than the truthvalues and value-ranges is not required by the axioms of the formal theory. Yet limiting the determination of the values of the primitive first-level functions to those objects qua arguments to which we can refer, at a later stage in the exposition of the concept-script, by using well-formed proper names - truth-value names, value-range names, definite descriptions, and complex and simple numerical terms - flies in the face of several remarks and fundamental stipulations that Frege makes in Grundgesetze.

It is almost beyond doubt that whenever Frege elucidates or defines a first-level function of his logical system he does so for all the objects there are as arguments and, hence, considers the first-order domain to be all-inclusive. I call this assumption B. So, it seems that in Grundgesetze we are facing a head-on conflict between $A$ and $B$. And it is this conflict which casts a gloom over Frege's attempt to endow value-range names with a unique reference beginning in $\S 3$ and apparently coming to a successful end in $\S 10-\S 12$. For clearly, the question of whether, by his own lights, he eventually succeeds in eliminating the pervasive indeterminacy of the references of value-range names by determining the values of the primitive first-level functions only for the truth-values and value-ranges as arguments depends crucially on the scope of the first-order domain. In what follows, I shall take a look at the evidence for assumption $B$ and comment also on its striking mismatch with $A$.

In Frege (1893), §2, Frege stresses (a) that the domain of what is admitted as arguments of first-level functions must be extended to objects in general ("Gegenstände überhaupt"). Let us call this demand "Frege's nonexclusiveness doctrine". (b) Correspondingly, he elucidates the primitive first-level functions of his system for all appropriate arguments (objects whatsoever), and (c) defines certain logically complex functions of first level, in accordance with his definitional principle of completeness, for all (possible) objects as arguments (see, for example, Frege (1893), p. 52). (d) Finally, the free object variables $a, b, \ldots$, which Frege employs in his formal theory, have the task of indicating objects in general, not those of a domain with fixed boundaries (cf. Frege (1903), p. 78). In what follows, I shall underpin (a), (b), and (c) by succinctly providing the relevant details.

To (a): In Frege (1893), §2, Frege argues that the range of function-values in mathematics cannot remain restricted to numbers if one acknowledges, for example " $\xi=4$ " and " $\xi>2$ " as names of proper functions as he does. However, towards the end of $\S 2$, he sets up the requirement mentioned above that the range of what is admitted as an argument of a first-level function must be extended to all objects whatsoever. Why? The requirement is not self-explanatory, especially for any reader who is not yet familiar with Frege's demand that functions (in this context those of first level), including concepts and 
relations, must have sharp boundaries. Instead of providing a rationale for extending the range of admissible arguments for first-level functions far beyond the initially proposed extension of the first-order domain (= the inclusion of truth-values, both as function-values and as arguments of first-level functions), Frege goes on to say: "Objects stand opposed to functions. Accordingly, I count as objects everything that is not a function, e.g., numbers, truth-values and the value-ranges to be introduced below."

To begin with, I assume that Frege uses the phrase "objects in general" not at random, but deliberately. It makes sense only if he wishes to include in the first-order domain all the objects there are. Trees, houses, and celestial bodies, for example, stand qua saturated entities as opposed to functions as, for example, numbers and truth-values. In this respect, there is no difference between concrete, spatio-temporal objects on the one hand and logical objects on the other. Moreover, the inference from the fact that Frege mentions only the kinds of logical objects that he needs for laying the logical foundations of number theory and real analysis, to the claim that he intends to narrow down the first-order domain to just those objects, is invalid. He uses the words "for example", and I am sure that he does so again deliberately. The upshot is that $\$ 2$, at least if it is taken au pied de la lettre, clearly speaks in favour of assumption $B$ saying that Frege regards the range of the first-order variables as all-inclusive, although he does not tell us why he is doing this.

To (b): In order to establish (b) it suffices to comment on Frege's elucidations of two primitive function-names. In $\S 5$, he introduces the first primitive function-name "—- $\xi$ ” of the concept-script (the horizontal) in such a way that $-\Delta$ is the True, if $\Delta$ is the True, and $-\Delta$ is the False if $\Delta$ is not the True. Note that he does not stipulate that $-\Delta$ is the False if $\Delta$ is the False. Nor does he stipulate that $-\Delta$ is the False if $\Delta$ is the False or a value-range. On the contrary, regarding the arguments of the first kind besides the truthvalues, he sets up a column other objects, which are all those that are distinct from both the True and the False.

Now, if Frege had intended to elucidate the primitive first-level function-names as well as the first-order universal quantifier of his system by reference to a strictly limited domain consisting only of the truth-values and value-ranges, he could have framed the elucidation, say, of the horizontal function, by saying that its value is the True for the True as argument and the False for the False and every value-range (that is not the True) as argument, and in this way he would have avoided any residual ambiguity. But he does not choose this way of phrasing. Thus, we may reasonably assume that the clause " $-\Delta$ is the False if $\Delta$ is not the True" is to mean not only, for example, "- $\Delta$ is the False if $\Delta$ is the False or the smallest prime number or the extension of the concept $\xi=\xi$ ", but also, for example, " - $\Delta$ is the False if $\Delta$ is the equator or the centre of mass of the solar system or the Moon or the Sun". In a footnote to $\$ 5$ (cf. footnote 3), Frege stresses that the stipulation concerning "— $\xi$ " is made in such a way that "_ $\Delta$ " refers to something under all circumstances if " $\Delta$ " refers to something. "Otherwise $-\xi$ would not be a concept with sharp boundaries, thus in our sense not a concept at all." So, his demand that the elucidation of " - $\xi$ " must ensure that "— $\Delta$ " refers to something under all circumstances amounts to or is tantamount to the requirement that the concept — $\xi$ must have sharp boundaries, and this means that for every object whatsoever as argument its value must be unambiguously determined.

The elucidation of the name of the conditional function in $\S 12$ deserves special attention in the context that I am considering. Unlike the elucidations of the first-level functionnames "— $\xi$ ", “ $+\xi$ " and " $\xi=\zeta$ ", it is preceded by Frege's identification of the True 
and the False with their unit classes in $\S 10{ }^{8}$ When in $\S 12$ Frege stipulates the values of the conditional function ${ }_{\zeta}^{\xi}$ for admissible arguments, he uses the phrase "any object that is not the True". Had he expressly decided at the outset of the exposition of his system to restrict the first-order domain to truth-values and value-ranges, it would have been straightforward, after having identified the truth-values with their unit classes, to choose the following elucidation of the conditional function: its value shall be the False (that is, $\left.\dot{\varepsilon}\left(\varepsilon=\tau^{\mathfrak{a}} \mathfrak{a}=\mathfrak{a}\right)\right)$ if $\dot{\varepsilon}(-\varepsilon)$ is taken as $\zeta$-argument and any value-range distinct from $\dot{\varepsilon}(-\varepsilon)$ is taken as $\xi$-argument; in the remaining cases-for example:

$$
\begin{aligned}
& {[\stackrel{\stackrel{\leftrightarrow}{\varepsilon}(-\varepsilon)}{\dot{\varepsilon}(\varepsilon=(\varepsilon=\varepsilon))} \quad(=\mathrm{T} \rightarrow \mathrm{T}),} \\
& {\left[\begin{array}{l}
\dot{\varepsilon}((-\varepsilon) \\
\dot{\varepsilon}(\varepsilon=
\end{array}\right.}
\end{aligned}
$$

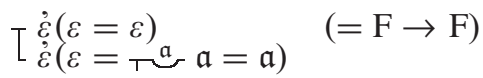

- the value of the conditional function shall be the True (that is, $\dot{\varepsilon}(-\varepsilon)) .{ }^{9}$ But again, Frege does not choose this way of phrasing, and I assume that he has good reasons not to use it. Admittedly, this observation does not furnish conclusive evidence for assumption $B$. But one can hardly deny that it speaks in favour of it rather than against it.

To (c): I hold that Frege defines certain logically complex first-level functions of his system for all objects whatsoever and I do so by relying on almost overwhelming evidence. A highly instructive example is his definition of the application operator " $\xi \cap \zeta$ " by

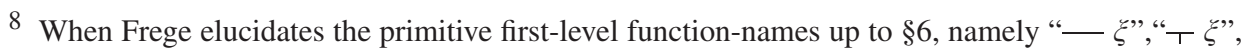
and " $\xi=\zeta$ ", concept-script terms that refer to admissible arguments for first-level functions and to the corresponding function-values are not yet available to him even if we charitably assume that at this stage of the exposition of the concept-script he would have been entitled to apply the rule which governs the insertion of a suitable argument expression into the argument-place of a function-name. Accordingly, Frege initially refers to the truth-values by using the metalinguistic terms "the True" and "the False" which he had already introduced in his essays "Function und Begriff' (1891) and 'Über Sinn und Bedeutung' (1892). Only after having elucidated the firstorder universal quantifier " $\mathfrak{a}-\varphi(\mathfrak{a})$ " in $\S 8$ would he have been able to form a concept-script name of the False such as " $\mathfrak{a}-\mathfrak{a}$ " or " $\mathfrak{a}-\mathfrak{a}$ " (provided again that insertion was explicitly recognized as a permissible syntactic operation). Furthermore, only by using such a name of the False could he have formed a concept-script name of the True. He could have done this by inserting, say,

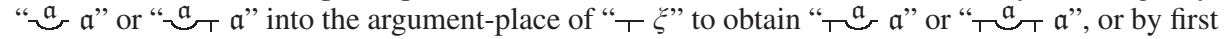
forming, say, " $\xi=\mathfrak{a}$ - $\mathfrak{a}$ " by insertion of " $\mathfrak{a}-\mathfrak{a}$ " into the $\zeta$-argument-place of " $\xi=\zeta$ " and then by forming, for example, "aㅢ $\mathfrak{a}=\mathfrak{a}-\mathfrak{a}$ " or " $\mathfrak{a}+\mathfrak{a}=\mathfrak{a}-\mathfrak{a}$ " again by insertion. In the concluding section of this essay, I shall argue that Frege's attempt to fix in a piecemal fashion the references of canonical-value range names uniquely $(\$ 3, \S 10-\$ 12)$ already miscarries for the simple reason that in Grundgesetze, $\S 10$ he is not yet in possession of the linguistic means that are required to make effectively the stipulation concerning the two truth-values. To be sure, at the stage of $\$ 10$ we know almost nothing about the syntax-the well-formed (nonprimitive) expressions - of the formal language.

9 In the light of what I said at the end of footnote 8, note that of the four value-range names which I use in the examples above only " $\dot{\varepsilon}(-\varepsilon)$ " seems to be available at the stage of Frege (1893), $\S 12$. Yet for the sake of convenience and simplicity, I felt free to ignore this fact in this special case. In his dual stipulation in $\S 10$, Frege elects " $\dot{\varepsilon}(-\varepsilon)$ " and " $\varepsilon\left(\varepsilon=T^{\mathfrak{a}}-\mathfrak{a}=\mathfrak{a}\right)$ " although at this stage in the exposition of the concept-script he is, strictly speaking, not justified in using " $\dot{\varepsilon}(\varepsilon=\uparrow \mathfrak{a}-\mathfrak{a}=\mathfrak{a})$ "; for details see 9.1 of this essay. 
using " $\ \xi$ " and second-order quantification. ${ }^{10}$ Especially Frege's subsequent explanations in Frege (1893), §34- $\$ 36$ are revealing as far as the range of admissible arguments for $\xi \cap \zeta$ is concerned. " $\xi \cap \zeta$ " is designed to create the possibility of representing both monadic and dyadic functions of first level by their value-ranges ("although of course not in such a way that they simply concede their places to them, for that is impossible"), and in this way to provide a device of using first-level functions instead of second-level functions in the system. Before setting up the definition " $a \cap u:=\backslash \dot{\alpha}(\neg \forall \mathfrak{g}(u=\dot{\varepsilon}(\mathfrak{g}(\varepsilon)) \rightarrow \neg \mathfrak{g}(a)=\alpha))$ " (I use here partly modern notation), Frege stresses that $\xi \frown \zeta$ must be explained (defined) for all possible objects as arguments. ${ }^{11}$ I take the phrase "for all possible objects as arguments" to mean: for all objects whatsoever as arguments. It cannot plausibly mean: for all valueranges as arguments. Here are my reasons. After having defined " $\xi \frown \zeta$ ", Frege gives a number of explications and closes $\S 34$ by summarizing:

two cases must be distinguished if the value of the function $\xi \cap \zeta$ is to be determined. If the $\zeta$-argument is a value-range, then the value of the function $\xi \cap \zeta$ is the value of that function whose value-range is the $\zeta$ argument for the $\xi$-argument as argument. If, on the other hand, the $\zeta$ argument is not a value-range, then the value of the function $\xi \cap \zeta$ is $\dot{\varepsilon}(\neg \varepsilon=\varepsilon)$ for every $\xi$-argument [that is, the extension of an empty concept].

Now, if Frege had intended to define $\xi \cap \zeta$ only for value-ranges and the two truth-values qua special value-ranges as arguments, he should have confined himself to stating what the

10 The term "application operator" was coined and introduced by Richard Heck (1997); see the discussion of this operator in Cook (2013), pp. A-29f. and Cook (2014).

11 It may seem that Frege introduces the primitive function-name " $\$ " into his logical system (cf. Frege (1893), §11) for the sole purpose of enabling him to define the application operator in such a way that it guarantees logical flexibility, austerity and conciseness in pursuit of the logicist project and, in particular, could be most effective in the projected proofs of the basic laws of cardinal arithmetic and real analysis. In the course of analyzing Frege's Grundgesetze-definitions, Cook and Ebert (2016) also comment on Frege's definition of the application operator. According to Cook and Ebert, this definition is the sole exception to what they call Frege's generalized recipe for identifying the objects falling under some mathematical concept $C$ (for details see pp. $325 \mathrm{ff}$.). All the other definitions of Grundgesetze are said to flow naturally from the generalized recipe. The latter emerges from the simple recipe by way of two modifications. The simple recipe is supposed to be Frege's methodological guideline when he comes to outline the logicist project in Grundlagen. The authors characterize it in four steps (for details see pp. 312ff.) and emphasize that in Grundgesetze Frege abandoned it in favour of the generalized recipe. As to the modifications which the generalized recipe involves, the first is the replacement of step 2 of the simple recipe ("Formulate the identity conditions for $C$ 's in terms of some appropriate equivalence relation $\Psi_{C}$ on the underlying domain of $\Phi_{C}$ 's") with a more general and flexible two step-process, while the second modification is the deletion of step 4 of the simple recipe ("Prove the relevant abstraction principle"). Cook and Ebert argue that Frege's definition of the application operator differs in character from the remaining definitions in Grundgesetze precisely because unlike those it requires an additional step: "... Frege needs a definition of ' $\cap$ ' that not only guarantees that the objects 'introduced' have the right identity conditions, but in addition that they are the right objects" (pp. 337f.). I recommend the reader of my essay to carefully study Cook \& Ebert (2016). It is a subtle, well-argued and stimulating investigation mainly of Frege's definitional methodology in Grundgesetze and related issues. Moreover, it sheds new light on what the authors call "the problem of the singleton". In \$8-\$9 of my essay, I shall critically discuss certain aspects of Frege's identification of the True and the False with their unit classes in Grundgesetze, $\S 10$, including the possibility of generalizing the stipulation " $\dot{\varepsilon}(\varepsilon=\Delta)=\Delta$ ", which he considers in a long footnote to $\$ 10$ but rejects. 
value of $\xi \cap \zeta$ is, if the $\zeta$-argument is a value-range. Yet he does not proceed in this way. "If, on the other hand, the $\zeta$-argument is not a value-range" cannot mean: "if the $\zeta$-argument is a truth-value". ${ }^{12}$ In the light of the identification of the True and the False with their unit classes, this would not make sense because the value of $\xi \cap \zeta$ for the two truth-values (their unit classes) as $\zeta$-argument is already determined via the stipulation of the first case: it is the value of that function whose value-range is the $\zeta$-argument for the $\xi$-argument as argument. Consequently, nor can it mean "if the $\zeta$-argument is a truth-value or any other object distinct from both the True and the False and any value-range". It can only mean: if the $\zeta$-argument is any object distinct from any value-range. I think that following Frege's line of argument in $\$ 34$ there is no point of vantage for rejecting my interpretation which also tallies with his initial statement that $\xi \cap \zeta$ must be explained for all possible objects as argument. ${ }^{13}$ Further corroboration of my thesis can be found in Grundgesetze, $\$ 35$, where Frege considers the case that as argument of the function $2 \backsim \xi$ an object that is not a valuerange is taken.

To sum up: If we rely on (a)-(d), we may justifiably assume that at least in some contexts which are of vital importance for Frege's system-primarily those which concern the elucidations and the definitions of first-level function-names-he considers the firstorder variables to range over all the objects there are. To respond to this by saying that the concept-script (presumably) does not contain names for, say, the Moon, the Great Wall of China or Julius Caesar, or that such spatio-temporal objects need not fall within the domain of a model of the axioms of the system would lack any force. For if the first-order domain comprises all objects whatsoever, then Frege faces the question and, indeed, has to answer it whether a value-range included in the domain coincides with the Moon or the Great Wall of China or Julius Caesar. The reason is that formulae of the form " $\forall x(x=\dot{\varepsilon} \Phi(\varepsilon) \rightarrow p)$ " will not have been endowed with a determinate reference, that is, a truth-value, unless " $x=\dot{\varepsilon} \Phi(\varepsilon)$ ", for the Moon or the Great Wall of China or Julius Caesar taken as value of " $x$ ", has been assigned a reference. Thus, if the domain of the first-order quantifiers is universal, if it comprises all the objects there are, then the combination of (i) the contextual stipulation in $\S 3$; (ii) the determination of the values of $\xi=\zeta$ for the True and the False as $\xi$-argument and a value-range as $\zeta$-argument, based on the identification of the True and the False with their unit classes in $\$ 10$; and (iii) the subsequent determination of the values of the description function and the conditional function for value-ranges in $\S 11$ and $\S 12$, fails to fix completely the reference of " $\varepsilon \varphi(\varepsilon)$ ". And since Frege defines the firstlevel cardinality operator in terms of the second-level value-range operator, the referential indeterminacy of the latter would be transmitted to the former and therefore to any term of an individual cardinal number to be defined in terms of the cardinality operator. Moreover, since Frege intended to define the real numbers as Relations of Relations ${ }^{14}$ (as ratios of magnitudes) and the complex numbers likewise as special value-ranges (cf. Frege (1893),

12 See in this respect also Frege (1902), p. 225.

13 There is a passage in Frege (1903, p. 74) in which Frege considers the possibility of restricting the scope of the first-order domain regarding, for example, a definition of a first-level relation. However, his remarks do not undermine my claim that in Grundgesetze he defines certain firstlevel functions for all objects whatsoever. This position is also forcefully advocated in Wehmeier (1999, 2015). See Blanchette's response to Wehmeier (2015) in Blanchette (2015).

14 The term "Relation" is Frege's shorthand expression for "Umfang einer Beziehung" ("extension of a relation"). Thus, in his logic Relationen (in English, I use "Relations" with a capital "R") are value-ranges of dyadic (first-level) functions whose value, for every pair of admissible arguments (objects), is either the True or the False. 
$\S 9)$, the referential indeterminacy of the value-range operator would likewise have affected any term that is assumed to refer to a real or a complex number.

The conflict to which Frege's wavering attitude towards the scope of the first-order domain gives rise appears even exacerbated when we focus our attention on Grundgesetze, $\S 31 .{ }^{15}$ As I mentioned earlier and shall argue in detail, the proof seems to rest crucially on the assumption that only the two truth-values are initially in the first-order domain. Once regular value-range names are recognized as being referential, the domain is considered to include value-ranges as well. And in the light of the twin stipulations in $§ 10$, which Frege expressly invokes in $\$ 31$, the domain is here apparently taken to consist only of valueranges - contrary to the evidence that we found elsewhere in Grundgesetze for assumption $B$. To be sure, if in $\S 31$ Frege did not make these assumptions about the first-order domain, he could not regard formulae of the form " $x=\dot{\varepsilon} \Phi(\varepsilon)$ " as having been endowed with a reference for every assignment of members of the domain to " $x$ ", and his proof would not even have got off the ground. In brief, I fail to see how the two positions regarding the scope of the first-order domain, which Frege seems to endorse in Frege (1893/1903), depending on what is at issue in the exposition of the formal theory, could be reconciled. The claim that he could not have been blind to this discrepancy and eventually decided to restrict the first-order domain once and for all to the truth-values and value-ranges hardly mitigates, let alone dispels the appearance of inconsistency. Thus, contrary to what some Frege scholars claim, the problem cannot be laid to rest.

I shall now turn to Grundgesetze, §26-\$31, with special emphasis on Frege's exposition in $\$ 29-\$ 31$. In order to understand his strategy in $\$ 29-\$ 31$ appropriately, we must first gain a clear idea about the syntax of the concept-script and its operating principles.

§3. Correctly formed names. The primitive function-names form the initial range of expressions of Frege's formal language and are of course well-formed on assumption. From these names we obtain by iterated application of interlocking formation rules both proper names and new function-names. The rules are of two kinds: (1) the rule of insertion (as I call it) licenses the formation of (a) complex proper names (function-value names) by inserting a fitting argument-expression into the argument-place of a monadic functionname of first or of second or of third level and (b) complex monadic function-names of first level by filling the $\zeta$ - or $\xi$-argument-place of a dyadic first-level function-name with a proper name. Frege calls insertion the first way of forming a name (cf. Frege (1893), §30); (2) the rules of gap formation (as I call them) govern the formation of (i) monadic firstlevel function-names, (ii) dyadic first-level function-names, and (iii) monadic second-level function-names with an argument-place of the second or of the third kind ${ }^{16}$ by removing some or all occurrences of a proper name either from a more complex proper namecase (i) in the standard form-or from a first-level monadic function-name-case (ii) or by removing some or all occurrences of a monadic first-level function-name from a proper name - case (iii) — and by marking the resulting gap(s) as an argument-place of

15 Concerning Frege's abortive attempt in $\$ 31$ to demonstrate that “ $\dot{\varepsilon} \varphi(\varepsilon)$ " has a reference, see the account especially in Heck (1997), Heck (2012), §3, §5 and Linnebo (2004). On Frege's proof of referentiality see also Thiel (1975), Resnik (1986), and Dummett (1991).

16 Argument-places of the first kind are suitable for the insertion of proper names, argumentplaces of the second kind are suitable for the insertion of monadic first-level function-names, and argument-places of the third kind are suitable for the insertion of dyadic first-level functionnames; cf. Frege (1893), §23. 
the appropriate kind (cf. Frege (1893), §26). Frege calls the extraction of function-names from more complex names via gap formation the second way of forming a name (cf. Frege (1893), §30). As to (i), note that the proper name that is removed from a given proper name will usually form a part of it. Yet Frege allows that the proper name we remove may also coincide with the original proper name. In this case, we obtain with " $\xi$ " the name of a oneplace first-level function whose value for any argument coincides with the argument itself. In such a special case, it would of course not make sense to speak of a complex proper name from which a simpler constituent proper name is removed. ${ }^{17}$

The application of the gap formation rules presupposes the prior application of the rule of insertion, in the first place to primitive function-names. ${ }^{18}$ According to the first and third rule, the gap formation starts from a complex proper name (function-value name), while according to the second rule it starts from a complex monadic function-name of first level. However, without applying the rule of insertion we cannot construct any function-value name from the primitive function-names and, consequently, cannot obtain any complex monadic function-name of first level. The first complex names of Frege's concept-script, namely certain function-value names, result from inserting a primitive one-place functionname of first or of second level into the argument-place of a primitive (monadic) functionname of second or of third level. ${ }^{19}$ Thus, in the order of construction (but only in this sense) insertion plays a more basic role in the syntax of the concept-script than gap formation.

It springs to mind that the process of gap formation is just the converse of forming names by insertion insofar as the latter relates to the formation of a proper name from a monadic function-name of first level and a monadic function-name of second level as well as to the formation of one-place first-level function-names from two-place first-level functionnames. The syntactic operation of insertion trivially implies that we always form a more complex name from two simpler names, whereas the application of a gap formation rule always goes hand in hand with the formation of a function-name that is less complex than the initial name from which one or more occurrences of a constitutent name are

17 Frege considered it to be unnecessary to state a gap formation rule that would govern the formation of (monadic) third-level function names. Two of the eight primitive function-names of his formal system are of third level. It is not clear to me why he introduces the ninth functionname " $\mathfrak{f}-\mu_{\beta_{\gamma}}(\mathfrak{f}(\beta, \gamma))$ " at all, since he says that he is not going to use it in his system. The eighth function-name is the second-order universal quantifier " $-\mathfrak{f}-\mu_{\beta}(\mathfrak{f}(\beta))$ ". As far as a gap formation rule that would govern the formation of names of dyadic second-level functions is concerned-equinumerosity between first-level concepts would be a prominent example of such a function-Frege likewise must have thought that such a rule (qua second-level analogue of the second gap formation rule) is not needed for the syntax of his formal language. Otherwise he could and would have included it without further ado in the set of gap formation rules.

18 It goes almost without saying that the formation of a function-name from primitive functionnames may involve not only an iterated application of the rule of insertion but also an iterated application of a gap formation rule.

19 Two examples: 1. By inserting " $-\xi$ " into the argument-place of " $\mathfrak{a}-\varphi(\mathfrak{a})$ " we form " $\mathfrak{a}-\mathfrak{a}$ ". 2. By filling the argument-place of " $\mathfrak{f}-\mu_{\beta}(\mathfrak{f}(\beta))$ " marked by " $\mu_{\beta}$ " with " $\mathfrak{a}-\varphi(\mathfrak{a})$ " we construct the truth-value name “ $\mathfrak{f}-\mathfrak{a}-\mathfrak{f}(\mathfrak{a})$ ”. In $\$ 23$, Frege distinguishes only between argument-places of the first, second, and third kind. The argument-place in a one-place third level-function-name such as " $\mathfrak{f}-\mu_{\beta}(\mathfrak{f}(\beta))$ " - " $\mu_{\beta}$ " is here suitable for the insertion of a monadic second-level functionname - could have been introduced as an argument-place of the fourth kind. Note that not only the letters " $\xi$ ", " $\zeta$ ", " $\varphi$ ", and " $\psi$ ", but also the argument-place markers " $\mu_{\beta}$ " and " $\mu_{\beta_{\gamma}}$ " are not to be regarded as concept-script signs. They serve Frege only provisionally. 
removed. If we take a closer look at the formation pedigrees, especially at those of some relatively complex function-names and proper names of the concept-script, we see that Frege designed the formation rules in such a way that they interlock like gear wheels in a gearbox. In fact, insertion and gap formation are in symbiosis.

Let me close this section by adding a final brushstroke to the picture I have painted so far of the syntax of Grundgesetze. It concerns the operation of gap formation and the combination of simplicity and complexity which it may involve. If gap formation is the last member of a formation sequence of a function-name, we may obtain not only complex function-names such as " $\frac{\zeta}{\zeta \zeta}$ " (which denotes disjunction by means of negation and the conditional), ${ }^{20}$ but also simple function-names such as " $\xi$ " or " $\xi=\xi$ " which refer to logically complex functions. Via iterated gap formation we could even go back from a multiply composed name to a primitive function-name, that is, to a name which Frege characterizes as logically simple.

The combination of simplicity and logical complexity which we encounter in cases like " $\xi$ " or " $\xi=\xi$ " has been largely ignored in the literature on the syntax and semantics of the concept-script. However, neither is the occurrence of this combination self-explanatory nor is it irrelevant in the present context. According to Frege's criterion of the simplicity of an expression (cf. Frege (1903), p. 79), the function-names " $\xi$ " and " $\xi=\xi$ " are simple because they are not put together from signs each of which has a reference (and a sense) on its own and contributes to determining the reference (and the sense) of the whole. Yet according to his use of the term "logically simple" as applied to concept-script names, " $\xi=$ $\xi$ ", for example, is not logically simple. The almost trivial reason is that it has not been singled out as one of the primitive function-names of the concept-script. While in Frege's view " $\xi=\zeta$ " expresses a simple sense due to its status as a primitive name introduced by elucidation, " $\xi=\xi$ " does not. Although he does not explain under what conditions the sense of an expression should be regarded as simple, the simplicity of the sense certainly implies that it is not further analyzable into simpler or more elementary senses. Every function-name of the concept-script that has been introduced by means of a definition is simple, but it is not logically simple. It refers to a logically complex function and expresses a complex sense. ${ }^{21}$ Yet it remains unclear how Frege could have explained the assumed complexity of the sense of a predicate like " $\xi=\xi$ ". Perhaps he would have suggested that the complexity derives from the fact that the sense is not immediately given by means of a standard elucidation which specifies the values of a primitive function for appropriate arguments but is obtained or grasped by running through the entire constructional history of " $\xi=\xi$ ". The formation of " $\xi=\xi$ " consists in a successive application of the rule of

20 The formation of " formed proper name " $\Delta$ ". In a second step, we insert " $T \Delta$ " into the $\zeta$-argument-place of the name of the dyadic conditional function and obtain a new one-place function-name of first level. In the third and final step, we apply to this the second gap formation rule and obtain " $-\xi$ ".

This example also shows that without forming a complex monadic first-level function name via insertion the second gap formation rule could not fulfil its role in the concept-script.

21 Dummett's claim (1981, pp. 294, 314, 539f.) that Frege did not intend his method of the extraction of function-names from more complex names via gap formation to apply to the formation of simple function-names is untenable. The mistake is probably due to the fact that he disregards Frege's distinction between simple and logically simple function-names. 
insertion (= first and second steps of construction) and the subsequent application of the first gap formation rule to " $\Delta=\Delta$ ". ${ }^{22}$

On the face of it, Heck (1997) seems to assume that in Frege's system gap formation serves only the formation of complex function-names. Heck writes: "What Frege allows us to do is to form what he calls a "composite' predicate..." (p. 440) by means of what I call gap formation. Later in his essay Heck seems to confirm this when he characterizes the induction step in Frege's argument as follows: "What Frege needs to show is that the two ways of forming more from less complex names preserve referentiality." As I pointed out above, the latter applies only to the formation of names by means of insertion qua last member of the formation sequence. The opposite applies to the extraction of functionnames via gap formation qua last member of the formation sequence. What Frege needs to show is precisely this: that (a) insertion (= the formation of more from less complex names) as well as (b) gap formation (= the formation of less from more complex names) preserve referentiality. ${ }^{23}$

\$4. Criteria of referentiality. Before moving from the syntax to the semantics of the concept-script, in particular to Frege's criteria and proof of referentiality and the relation they bear to the formation rules, it will be useful to fix some terminology. In the remainder of this essay, including this section, I shall frequently employ the term "truth-value name". As I already mentioned in the Introduction, I shall argue that truth-value names play a key role in Frege's proof of referentiality for his concept-script in $\S 31$ and, in particular, that in his view value-range names only have a reference in the context of a truth-value name (sentence). Although in Grundgesetze Frege does not mention the context principle of Grundlagen, let alone comments on its supposed role in his account of the referentiality of proper names and function-names, he crucially relies on it. So, as we shall see, especially regarding the semantics of abstract singular terms, there is a still a close relationship between Grundlagen and Grundgesetze.

In the exposition of the concept-script, Frege does not use the term "truth-value name" at all-he always uses the phrase "name of a truth-value"-but the introduction of the former while retaining the latter helps to avoid ambiguity in certain contexts, notably in $\S 31$ where he assumes that names of a truth-value (= truth-value names) refer to something before carrying out the first step in his proof of referentiality. By a truth-value name I suggest that we understand the name of a concept-value or relation-value for appropriate arguments, that is, a proper name à la Frege which in the last stage of its formation, beginning with primitive function-names, is always formed by inserting an admissible argument-expression or admissible argument-expressions into the argument-place(s) of a concept- or relation-expression. In short, a truth-value name is a proper name that has the syntactic structure of a declarative sentence and expresses a thought- not to be conflated

22 " $\Delta$ " is used here as an auxiliary name which represents a well-formed proper name of the conceptscript. As we have seen, once Frege has introduced the first-order universal quantifier " $\mathfrak{a}-\varphi(\mathfrak{a})$ ” (Frege, 1893, §8) and feels entitled to apply the rule of insertion (which he characterizes as late as in $§ 30)$, he is able to form concept-script proper names by filling the argument-place of “ $\mathfrak{a}-\varphi(\mathfrak{a})$ " with a primitive monadic function-name of first-level.

23 If Heck wants to convey that Frege must show that the two ways of forming nonprimitive names from the logically simple function-names preserve referentiality, then his way of formulating this is probably misleading. To reemphasize, not every function-name that we obtain by means of gap formation in the last step of its construction is to be regarded as a complex name in the light of Frege's criterion of the simplicity (or complexity) of a name. 
with a concept-script sentence (Begriffsschriftsatz) " $-\Delta$ " which consists of a truth-value name or a Roman marker of a truth-value with a judgement stroke prefixed. The rationale for introducing the term "truth-value name" is the fact that not every name of a truth-value of the concept-script is a truth-value name. Thanks to certain stipulations, Frege is able to refer to the True (= its own unit class), for example, not only by using, say, " $\mathfrak{a}-\mathfrak{a}=\mathfrak{a}$ ", but also by using a value-range name such as " $\varepsilon(\varepsilon=(\varepsilon=\varepsilon))$ " or a definite description such as "\} \hat { \varepsilon } ( - \varepsilon ) \text { ". Note that unlike the first name the two latter names do not express a thought. } But of course they express a nonpropositional sense. This anomaly is a consequence of Frege's stipulations in Frege (1893), §10-\$11.

At the beginning of $\S 28$, Frege lays down as a fundamental principle of his conceptscript that correctly formed names must always refer to something. He then goes on to explain the conditions that must be satisfied for calling a concept-script name correctly formed and to state in $\$ 29$ the criteria of referentiality for concept-script-names. For the sake of perspicuity, I present the criteria in a formalized version and use " $(\mathbb{B})$ "as a semantic predicate denoting the property of having a reference or of being referential.

(A) $(\mathrm{B}) \Phi(\xi) \leftrightarrow \forall \Delta(\mathbb{B} \Delta \rightarrow \mathbb{B} \Phi(\Delta))$.

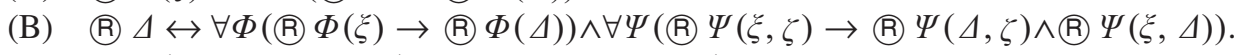

(C) $\mathbb{B} \Psi(\xi, \zeta) \leftrightarrow \forall \Gamma \forall \Delta(\mathbb{B} \Gamma \wedge$ ( $\Delta \rightarrow \mathbb{B} \Psi(\Gamma, \Delta))$.

(D) $\mathbb{B} \Omega_{\beta}(\varphi(\beta)) \leftrightarrow \forall \Phi(\AA) \Phi(\xi) \rightarrow$ ( $\left.\Omega_{\beta}(\Phi(\beta))\right)$.

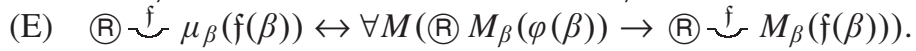

Generally speaking, the characteristic mark of these criteria can be described as follows. A name to be tested or shown to be referential (let us call it test name)-(a) one-place function-name of first level, (b) proper name, (c) two-place function-name of first level, (d) one-place function-name of second-level, and (e) one-place function-name of third levelhas a reference, if from the assumption that all expressions of an appropriate syntactic category associated with (a), (b), (c) (d), and (e) (let us call them base names) - namely ( $\mathrm{a}^{\prime}$ ) proper names, $\left(b^{\prime}\right)$ monadic and dyadic first-level function-names, $\left(c^{\prime}\right)$ proper names, $\left(d^{\prime}\right)$ monadic first-level function-names, and $\left(\mathrm{e}^{\prime}\right)$ monadic second-level function-names with an argument-place of kind 2-refer to something, it follows that the complex names that we obtain by combining via insertion the test names with the base names have a reference. In a nutshell, a test name has a reference if certain more complex names, of which it forms a part, refer to something. Criterion (B) differs structurally from the other criteria in two respects: (i) the proper name to be shown to be referential functions itself as a substitution instance for the base names, namely the associated monadic and dyadic function-names; (ii) expressions of both fundamental logical types result here from insertion: proper names and (monadic first-level) function-names. By contrast, the simple supplementation of (a) by a base name ( $\left.a^{\prime}\right)$ according to (A), of (d) by a base name ( $\mathrm{d}^{\prime}$ ) according to (D), of (e) by a base name ( $\left.\mathrm{e}^{\prime}\right)$ according to (E) as well as the double supplementation of (c) by two base names $\left(\mathrm{c}^{\prime}\right)$ according to $(\mathrm{C})$ lead exclusively to the formation of function-value names. I call the names formed by means of insertion

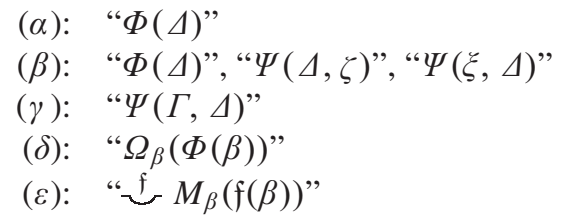

target names for the test names (a)-(e). 
Three further comments are in order here.

To begin with, I assume that when stating these criteria, Frege is focusing entirely on well-formed concept-script expressions of all three classes mentioned above: test names, base names, and target names. There is not even a trace of textual evidence that he has any auxiliary names in mind. In $\S 5$ (footnote 3 ), Frege emphasizes that he uses the capital Greek letters " $\Gamma$ " and " $\Delta$ " as if they were names referring to something (to an object), without specifying their reference. He adds that they will not occur in the "Begriffsschriftentwicklungen" (literally: in the development of the concept-script), just as little as " $\xi$ " and " $\zeta$ ". In saying this, he is tacitly referring to part II of Frege (1893) entitled "Proofs of the Basic Laws of Cardinal Number". As a matter of fact, not only " $\Gamma$ " and " $\Delta$ ", but also names like " $\Phi(\xi)$ " and " $\Psi(\xi, \zeta)$ " are only used in part I of Grundgesetze entitled "Exposition of the concept-script". Although to my knowledge Frege does not say anything specific about the status of " $\Phi(\xi)$ " and " $\Psi(\xi, \zeta)$ ", he most likely treats them as names that are on a par with " $\Gamma$ " and " $\Delta$ ", that is, he uses " $\Phi(\xi)$ " and " $\Psi(\xi, \zeta)$ " as if they were names referring to something (to any monadic or dyadic first-level function) without stating their reference.

In my view, it would hardly make sense to apply, say, criterion (B) to " $\Gamma$ " and " $\Delta$ ", since the reference of such names is only conditional and unspecified. They are treated as if they were referential without indicating what their reference is. It is true that in carrying out his proof of referentiality Frege does employ auxiliary names, but, as we shall see more clearly in $\$ 7$, he does so only for the sake of convenience. Admittedly, my claim that the criteria of referentiality are intended to apply only to concept-script names, could appear to be at odds with Frege's view that a first-level function-name has a reference only if the corresponding function has a value for every argument or every pair of arguments of an all-encompassing first-order domain. Yet we should not infer from his requirement of the sharp delimitation of (first-level) concepts and relations that the criteria are indeed intended to apply to auxiliary names; nor should we expect that by using such names for the sake of convenience he can effectively alleviate the conflict between the requirement and the limited availability of proper names in the concept-script, namely only of those that refer to truth-values and value-ranges and not to any other object. ${ }^{24}$

Second, the criteria (A)-(E) are obviously affected by circularity. It is therefore doubtful whether their application to a given range of concept-script names can ensure that every such name has a unique reference. Although Frege was aware of the circularity of (A)(E), it seems that he did not carefully assess the consequences that it had for his proof of referentiality. "These sentences are not to be construed as explanations of the words 'to have a reference' or 'to refer to something', since their application always presupposes that one has already recognized some names as referential; but they can serve to extend the domain of such names gradually" (Frege, 1893, p. 46). It follows from (A)-(E), "that every name formed from referential names refers to something. This formation proceeds in such a way that a name fills argument-places of another name that are fitting for it" (Frege, 1893, p. 46). Now, it is undeniable that the insertion of a referential name into

24 Linnebo (2004) discusses the "auxiliary", the "nonauxiliary", and the "mixed" reading of the criteria of referentiality. He argues that Frege understood the criteria relating to proper names (the regular value-range names) and those relating to function-names (at least in the case of the first four primitive names that Frege introduces into his system) in the "nonauxiliary" way. However, I doubt that the use of auxiliary names was a big issue for Frege. The textual evidence suggests that it was only a convenient device for him. After having introduced the device in Frege (1893), §5, he does not address it anymore. This is not to say that it is irrelevant for assessing Frege's criteria and proof of referentiality, but in my view both Heck (1997) and Linnebo (2004) make perhaps too much of it. See Heck's detailed response to Linnebo's analysis (Heck, 2012, §5.2, pp. $121 \mathrm{ff}$.). 
the argument-place(s) of a referential function-name, which is the only mode of formation that Frege mentions here, always produces a (more complex) referential name. Let " $a$ " and " $b$ " be well-formed proper names of the concept-script and suppose that $\mathbb{B} a$, (B) $b$ and $\AA \Psi(\xi, \zeta)$ hold. According to criterion $(\mathrm{C}), \AA \Psi(\xi, \zeta)$ is then equivalent with

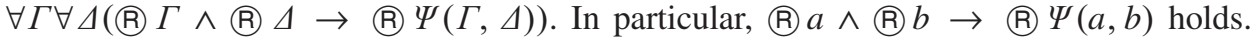
And since the antecedent holds on assumption, we obtain $\AA \Psi(a, b)$ by applying modus ponens. ${ }^{25}$ The upshot is that the criteria of referentiality, which Frege wishes to employ as inductive clauses in his proof of referentiality, in fact guarantee that all names which result from the primitive function-names via insertion refer to something, if the primitive names have a reference. Yet they cannot ensure that those names whose construction from the primitive names involves a single or an iterated application of a gap formation rule are referential, if the primitive names are referential. In the first half of $\S 30$, Frege comments on insertion, and then goes on to mention a second possibility (besides insertion) to form firstlevel function-names, namely by applying gap formation. He does not explain, however, how this second syntactic operation is supposed to relate to the previously stated criteria of referentiality. He observes only that the first-level function-name that we obtain by means of gap formation always has a reference, if the primitive names from which it is formed are referential. Frege adds that the extracted function-name can in turn be used to form referential names in the first or second way, that is, via insertion or gap formation.

For some reason, Frege designed the criteria of referentiality in such a way that the more generally applicable method of function-name formation, namely gap formation, is left out of account. Perhaps he took it for granted that gap formation, since it always leads to less from more complex names, preserves referentiality. If a given proper name or complex function-name from which we remove some or all occurrences of a constituent name is referential, then the function-name that we obtain must have a reference, since it formed a part of the initial more complex name. But here as well in a few other places in Grundgesetze, $\S 10, \S 29-\$ 31$ we can only speculate.

Third, a comment on the role of Frege's context principle in the criteria of referentiality is in order, especially with an eye to the main argument that I shall present in $\$ 7$ of this essay. It is common ground that in Grundlagen Frege had not yet drawn a terminologically strict distinction between reference (Bedeutung) and sense (Sinn) but was rather using an undifferentiated notion of meaning. ${ }^{26}$ There is, however, ample evidence that his main concern with the context principle in Grundlagen "Only in the context of a sentence do words have a meaning" was to apply it to the references of words, in particular to the references of abstract singular terms such as "the direction of line $a$ " or "the cardinal number that belongs to the concept $F " .{ }^{27}$ Construed as a principle concerning reference, it states the general condition that a word must satisfy in order to have a determinate reference at all. Thus interpreted, the context principle embodies the primacy of sentence-reference over word-reference in the following sense: the reference of a subsentential expression consists in its contribution to determining the reference of a sentence in which it may occur.

Taken at face value, the criteria of referentiality appear as a generalized version of Frege's context principle from Grundlagen regarding reference. The target names figuring in the criteria (A) and (C) as well as the target names of the first kind of criterion (B)

25 Cf. also Thiel (1975), p. 146.

26 As to Frege's semantic terminology in Grundlagen see Schirn (2010), pp. 51f.

27 Here is not the place to analyze Frege's context principle in Grundlagen in any detail; this would require a separate article. Suffice it to characterize briefly its quintessence regarding reference. 
are not expressly specified as truth-value names; they could belong to another subcategory of proper names. Solely in the case of criterion (E), which governs the only third-level function-name that Frege uses in his logical system, namely the second-order universal quantifier, is the target name always a truth-value name. However, if prior to an application of the criteria truth-value names are assumed to be referential in their own right-and Frege must in any application of them presuppose that some names are already recognized as referential - the situation is as follows: the demonstration that a function-name qua test name (a), (c), (d) or a proper name qua test name (b) other than a truth-value name is referential, can only be accomplished if the target names are semantically independent names, that is, truth-value names. In saying this, I assume that from Frege's point of view truth-value names of the concept-script are semantically independent regarding reference precisely in the sense that, unlike proper names of any other subcategory, they do not have a reference only on condition that more complex names, in which they occur, are referential. (And in contrast to proper names of any other subcategory, they do express a thought.) Plainly, it would hardly make sense to state, by appeal to criterion (B), that a truth-value name has a reference, if more complex proper names and monadic first-level function-names, in which it occurs, are referential. Nor would it help to narrow down the range of target names for a truth-value name qua test name to truth-value names because in that case we may face an infinite regress. For clearly, any attempt to set up an upper bound regarding the complexity of a truth-value name qua target name for a truth-value name qua test name would founder on the arbitrariness and randomness of our actual choice. In short, the reference of a truth-value name does not consist in the contribution it makes to determining the reference of a more complex truth-value name; it just consists in being either the True or the False. I conclude then that the criterion of referentiality (B) for proper names is not intended to apply to truth-value names at all or in other words: truth-value names do not themselves figure as test names regarding referentiality. ${ }^{28}$

To spell this out again in more detail. Since any monadic first-level function-name that results from a dyadic first-level function-name qua base name $\left(b^{\prime}\right)$ by means of single insertion according to criterion (B) is a semantically dependent target name $(\beta)$ for a test name (b), $(\beta)$ must in turn be shown to be referential according to criterion (A) in order to secure a reference for (b). Again in a nutshell, an application of criterion (B) must always be succeeded by an application of criterion (A). Whether a target name $(\beta)$ of the form " $\Phi(\Delta)$ " and whether a target name $(\alpha),(\gamma)$ and $(\delta)$ must in turn be shown to be referential according to criterion (B) in order to establish that a test name (b) or (a), (c), (d) has a reference depends on the question of whether it is a truth-value name or not. The reconstruction and analysis of Frege's attempted demonstration that " $\dot{\varepsilon} \varphi(\varepsilon)$ " is referential will show this clearly.

Michael Dummett's extensive discussion in Dummett (1973) and Dummett (1981) of Frege's alleged abandonment of the context principle after 1891 is not a paragon of clarity, and what is worse, it is wrong-headed. In both studies, he attempts to convince us that Frege's assimilation of declarative sentences to proper names is in sharp conflict with the

28 In the course of dealing with Russell's Paradox, Frege discusses the possibility of regarding both class-names and numerals as pseudo proper names, as parts of signs which have a reference only as wholes (cf. Frege (1903), p. 255), and in this connection refers to Frege (1893), §29. On reflection, he rejects this route as impassable. However, I find his reference to $\$ 29$ in the relevant context puzzling. It does not follow from his criteria of referentiality that proper names and function-names are merely "syncategorematic" signs of more complex signs of which they form a part. 
context principle and that it is for this reason that it no longer appears in Frege's writings after 1891. He writes (Dummett, 1973, pp. 644f.):

The most disastrous effect of the new doctrine was the abandonment of one of Frege's most important insights, that of the central role of sentences in the theory of meaning. If sentences are only a special kind of proper name, then the senses of other expressions cannot consist in the contribution which they make to determining the senses of sentences, in particular, in which they occur: the most we can say is that the sense of an expression is directed towards the determination of the sense of a complex name in which it may occur.

The if-then sentence in this quotation contains a non sequitur and the last sentence in it is false. Dummett overlooks here the fact that the assimilation in question by no means deprives declarative sentences of their unique status in Frege's theory of sense. Due to the assimilation, in Grundgesetze sentences qua truth-value names in fact belong to the same logical type as proper names of other subcategories (value-range names, definite descriptions, numerical terms). In the syntax of the concept-script, this is shown by the fact that truth-value names may fill the same argument-places as proper names of all other subcategories that can be constructed in the formal language or are introduced in it by means of a definition, namely argument-places of the first kind. By contrast, in the realm of sense, the assimilation has no essential impact, contrary to what Dummett claims. In particular, it does not affect the fact that in the formal language every well-formed truthvalue name has the unique property of expressing a thought. Frege is therefore entitled to state, quite in the spirit of the context principle as a principle relating to sense, that the simple or complex names of which a name of a truth-value (= truth-value name) consists, contribute to expressing the thought, and that this contribution of the individual name is its sense (cf. Frege (1893), §32).

In Dummett $(1973,1981)$ and also in Dummett (1991), Dummett draws a similar conclusion from the assimilation under discussion for the realm of reference and sees in Frege's strategy(ies) in Grundgesetze, $\S 10$ and $\$ 31$ only a generalization, an "echo" of the original context principle regarding reference, but hardly "an intelligible doctrine". If my line of argument in the subsequent sections carries conviction, then Dummett's assessment of the context principle in Grundgesetze concerning reference can hardly be maintained. ${ }^{29}$

§5. Getting the proof in $\$ 31$ off the ground. At the outset of his proof in $\S 31$, Frege resumes the idea stated at the end of $\S 30$ : in order to show that proper names and first-level function-names, that can be formed from the primitive names by applying the formation rules of the two kinds, refer to something he must only demonstrate that the primitive names are referential. Note that besides mentioning proper names and nonprimitive first-level function-names Frege fails to mention nonprimitive monadic second-level function-names. Unlike the former function-names, where the last member of the formation sequence can be either insertion or gap formation, the latter always result, in the last stage of their construction from primitive names, from gap formation, namely by removing some or all occurrences of a monadic first-level function-name from a

29 Let me mention that in Dummett (1991) Dummett concedes, although without referring to his earlier work, that in $\$ 32$ of Grundgesetze "Frege continued to allot a primacy to sentences, with respect to sense" (p. 210). 
proper name and by marking the resulting gap(s) as an argument-place of the appropriate kind. $^{30}$

Frege proceeds from the assumption that the names of truth-values are referential. Contrary to what Heck (1997, p. 468) suggests, Frege is here most likely referring to a syntactic subcategory of proper names, namely to truth-value names in the sense in which I explained this term in the preceding section. ${ }^{31}$ Let me call this Frege's assumption of propositional referentiality or more briefly Frege's initial assumption in $\$ 31$. Again, I further assume that he has only truth-value names of the concept-script in mind. The value-range terms " $\dot{\varepsilon}(-\varepsilon)$ " and " $\dot{\varepsilon}\left(\varepsilon=\tau^{\mathfrak{a}} \mathfrak{a}=\mathfrak{a}\right)$ ", for example, as well as the definite descriptions "\} \hat { \varepsilon } ( - \varepsilon ) \text { " and "\} \grave { \varepsilon } ( \varepsilon = \tau ^ { \mathfrak { a } } - \mathfrak { a } = \mathfrak { a } ) \text { ", for example, are not included in Frege's initial } assumption, although by virtue of his stipulations in $\S 10-\$ 11$ all four names refer to a truth-value (including those that are coreferential either with the first or the second member of each pair of names and belong to the same syntactic category as these). Suppose that at the outset of his proof of referentiality Frege considered " $\varepsilon(-\varepsilon)$ ", for example, to be referential outright on the ground that it is a name of the True. Clearly, in this case there would be no point in showing it to be referential according to criterion (B). Yet this is

30 This follows from the formation rules for the formal language of Grundgesetze. The rule of insertion, as conceived of by Frege, does not license the insertion of a first-level functionname into one of the two argument-places of a dyadic second-level function name. And, as I pointed out earlier, he does not state a gap formation rule that allows the formation of a dyadic second-level function-name by removing some or all occurrences of a first-level function-name from a monadic second-level function-name.

31 Textual evidence for my claim can be found especially in two statements that Frege makes at the beginning and at the end of $\$ 32$ : "Every such name of a truth-value expresses a sense, a thought... Now, the simple or complex names of which a name of a truth-value consists contribute to expressing the thought, and this contribution of the individual name is its sense. If a name is part of the name of a truth-value, then the sense of the former is part of the thought expressed by the latter." Plainly, these statements make only sense if "name of a truth-value" is meant in the sense of "truth-value name" (sentence). Heck (1997) thinks that he has an argument for his claim that Frege's assumption at the outset of $\$ 31$ is not meant to apply (only) to a syntactic category of proper names, namely to sentences. "But this [= the opposite claim] cannot be right. Some of [Frege's] arguments accord with such a reading. But his discussion of the universal quantifier does not. Anything of the form ' $\forall x . \Phi(x)$ ' is certainly a sentence: so, if what Frege were assuming was that all sentences denote, he could simply have noted that fact and been done with it. But he does not proceed in that way" (Heck, 1997, p. 468, footnote 21). This argument is hardly compelling. It is true that Frege fails to explain why in the case of the first-order universal quantifier he does not, similar to his way of previously establishing that " $-\xi$ —, " $-\xi$ ", " $\xi=\zeta$ " and " ${ }_{\zeta} \xi$ " are referential, confine himself to stating the simple fact: whenever a function-name “ $\Phi(\xi)$ " refers to something, it follows that " $\mathfrak{a}-\Phi(\mathfrak{a})$ " is referential, since it is a name that refers to something (namely a truth-value) on assumption, whatever the reference of " $\Phi(\xi)$ " is. Similar remarks would apply mutatis mutandis to his treatment of the second-order quantifier. Yet it seems clear that Frege could again have chosen such an explanation without offending against any of his insights into the nature of the referentiality of concept-script names. We can after all only speculate about the reason why he chose the more detailed explanation in the case of the firstorder and second-order universal quantifiers, but should not jump to conclusions from his choice.

On the one hand, we have already come across " $\varepsilon(-\varepsilon)$ " in $\$ 10$, just one section after the introduction of the value-range notation. On the other hand, in $\$ 31$ we are informed that with the introduction of " $\varepsilon \varphi(\varepsilon)$ " new proper names are simultaneously introduced. One might wish to object that at the stage of $\S 31$ we are already familiar with value-range names from previous sections. So, what is new about them? I shall comment in more detail on this apparent discrepancy in $\S 6$. 
precisely what Frege thinks he must do for " $\varepsilon(-\varepsilon)$ " and for regular value-range names in general in order to succeed in demonstrating that " $\varepsilon \varphi(\varepsilon)$ " has a reference.

Every well-formed truth-value name of the concept-script is a proper name that possesses the syntactic structure of a declarative sentence and, if all its constituent expressions have a sense, expresses a thought that, if true, can be acknowledged in a judgement as true. You will also recall that a concept-script sentence consists of a truth-value name or a Roman marker of a truth-value ${ }^{32}$ with a judgement stroke prefixed. Of course, the judgement stroke must always be accompanied by the horizontal in order to exercise its function. The thought expressed by the truth-value name is that the conditions under which it refers to the True are fulfilled. Furthermore, every well-formed truth-value name of the formal language refers by its very nature to one of the two primitive objects of logic. This applies only to some of the value-range terms and definite descriptions of the conceptscript, and only thanks to certain stipulations that Frege makes. I think that it is mainly due to these distinctive features of truth-value names and the ones that I mentioned at the end of $\S 4$ that Frege chooses them as a class of proper names that should be regarded as referential in their own right, before the first step in the proof of referentiality is carried out. However, in doing this he must have been aware that every genuine truth-value name of the concept-script-and not just an auxiliary name " $\Delta$ " that, for the sake of convenience, he may wish to construe as representing a truth-value name in the exposition of his logical theory-must be formed from the primitive function-names by (iterated) application of the formation rules. To reemphasize: " $\Gamma$ " and " $\Delta$ " do not belong to the concept-script. Hence, the presupposition that truth-value names are referential in their own right and $a b$ initio already implies to a large extent what Frege intends to show in $\$ 31$, namely that the primitive function-names of the concept-script have indeed a reference, assuming that the formation rules transfer the property of being referential to all well-formed nonprimitive expressions.

We are now prepared to take a closer look at the details of the proof of referentiality. ${ }^{33}$ What I already mentioned earlier will now become obvious, namely that the strategies which Frege applies in $\S 10$ and in $\S 31$ are inseparably interwoven. In the case of the

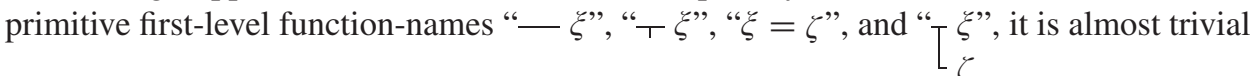

to demonstrate that they have a reference. Frege must only show that the target names that result from inserting a truth-value name qua base name into the argument-place of the primitive monadic function-names qua test names, and truth-value names qua base names into the argument-places of the primitive dyadic function-names qua test names, are referential. The resulting names are truth-value names and on assumption referential. At this stage of the proof, Frege apparently assumes that only the True and the False are in the first-order domain. Accordingly, he believes that it suffices to insert names of truth-values into the argument-place(s) of the primitive first-level function-names, since

32 However, such a marker is transformed into a truth-value name by using German letters in place of Roman letters prefixed with "concavities" (cf. Frege (1893), §32).

33 I assume that Frege's proof of referentiality was intended to secure also a sense for every wellformed name of the formal language: "Thus it is shown that our eight primitive names have a reference and thereby that the same holds for all names correctly formed from them. However, not only a reference but also a sense belongs to all names correctly formed from our signs" (Frege, 1893, p. 50). Although Frege does not expressly state that it is thereby shown that all names correctly formed from the primitive names have a sense, it seems rather likely that he construes the proof of referentiality at the same time as a demonstration that all well-formed names are endowed with a sense. 
"here we do not yet know other objects". However, this remark strikes me as awkward. The reader of Grundgesetze should have thought that at the end of $\S 12$ the references of value-range names are already completely determined and, hence, are sufficiently known as logical objects. If that were not so, then Frege would not have had the right to present the formal concept-script version of Basic Law V in $\$ 20$. In $\$ 20$, he observes: "we saw in $\S 3, \S 9$ that an identity of value-ranges may always be transformed into the generality of an identity, and conversely: $\vdash(\dot{\varepsilon} f(\varepsilon)=\dot{\alpha} g(\alpha))=(\mathfrak{a}-f(\mathfrak{a})=g(\mathfrak{a}))$." At this stage of the exposition of the concept-script, he must assume that by virtue of his stipulations in $\S 3, \S 9-\S 12$ he has in fact succeeded in conferring a complete reference on " $\dot{\varepsilon} \varphi(\varepsilon)$ ". And he must of course further assume that each of the other primitive names occurring in the concept-script version of Basic Law V-namely "— $\xi$ ”, " $\xi=\zeta$ ”, and " $\mathfrak{a}-\varphi(\mathfrak{a})$ "—-has likewise been endowed with a unique reference via its elucidation. ${ }^{34}$ Otherwise, the truthvalue name " $(\dot{\varepsilon} f(\varepsilon)=\dot{\alpha} g(\alpha))=(\mathfrak{a}-f(\mathfrak{a})=g(\mathfrak{a}))$ " would lack a determinate reference and, hence, would not express an axiom at all and could consequently not appear with "- prefixed.

When in his proof of referentiality Frege turns to the first-order universal quantifier, he does not confine himself to stating that for every referential function-name " $\Phi(\xi)$ ", "a $-\Phi(\mathfrak{a})$ " is a name of a truth-value and therefore referential. Rather he first mentions the criterion of referentiality (A) for monadic first-level function-names and then concludes from the assumption that " $\Phi(\xi)$ " satisfies (A) that the target name " $-\mathfrak{a}-\Phi(\mathfrak{a})$ " for "a $-\varphi(\mathfrak{a})$ " has a reference, from which it follows according to criterion (D) that the test name " $\mathfrak{a}-\varphi(\mathfrak{a})$ " is referential. Frege writes:

In order to examine whether the name of the second-level function, ' $\mathfrak{a}-\varphi(\mathfrak{a})$ ', refers to something, we must ask whether, whenever a function-name ' $\Phi(\xi)$ ' refers to something, it follows that ' $\mathfrak{a}-\Phi(\mathfrak{a})$ ' is referential. Now, ' $\Phi(\xi)$ ' has a reference if, for every referential proper name ' $\Delta$ ', ' $\Phi(\Delta)$ ' refers to something. If so, this reference is either always the True (whatever ' $\Delta$ ' may refer to), or not always. In the first case, ' $\mathfrak{a}-\Phi(\mathfrak{a})$ ' refers to the True; in the second, to the False. Thus, whenever an inserted function-name ' $\Phi(\xi)$ ' refers to something, it follows that ' $\mathfrak{a}-\Phi(\mathfrak{a})$ ' refers to something.

One question concerning this passage immediately forces itself upon us: what does Frege mean here precisely by the phrase "for every referential proper name " $\Delta$ "'? One thing I take to be obvious. Frege definitely did not think that every object has a name, let alone that his concept-script contains a name for every object. And if I am right in assuming (a) that in $\$ 31$ he initially considers the first-order domain to contain only the True and the False and (b) that in the proof of referentiality he employs auxiliary names like " $\Gamma$ " and " $\Delta$ " only for the purpose of representing any well-formed proper name of the conceptscript that is available at a certain stage of the exposition, then "for every referential proper name ' $\Delta$ '" can only mean: for every truth-value name of the concept-script. Before Frege turns to the function-name " $\dot{\varepsilon} \varphi(\varepsilon)$ " and attempts to demonstrate that it refers to something, truth-value names are the only proper names that are regarded as being referential; and they are so regarded before the first step of the proof is carried out. Thus, the interpretation that I suggest seems to tally with the way Frege actually proceeds in his proof before he comes to examine the semantics of " $\dot{\varepsilon} \varphi(\varepsilon)$ ". Moreover, it seems that my interpretation would render

34 It is characteristic for the concept-script versions of Frege's six Basic Laws that they contain only primitive names. 
his account coherent in the context of $\$ 31$. However, due to the lack of conclusive textual evidence and especially in the light of Frege's clashing assumptions concerning the scope of the first-order domain, I do not wish to vouch for this interpretation.

§6. The name of the value-range function: the introduction of a new function-name and new proper names in \$31. When Frege turns to the other primitive second-level function-name of the formal language, namely " $\varepsilon \varphi(\varepsilon)$ ", he says that it is less simple to show that it is referential than in the previous case of " - $\xi ", "+\xi ", " \xi=\zeta$ ", " $T \begin{aligned} & \xi ", \\ & \zeta\end{aligned}$ “a- $\varphi(\mathfrak{a})$ " and " $\mathfrak{f}-\mu_{\beta}(\mathfrak{f}(\beta))$ ". The main reason for the increase in complexity is supposed to be this: unlike the introduction of the six function-names above, the introduction of " $\varepsilon \varphi(\varepsilon)$ " goes hand in hand with the introduction of new proper names (value-range names), not only for the primitive monadic function-names of first level that he had already elucidated in $\S 5, \S 6, \S 11$, but also in advance for any such name that may yet be introduced.

On the face of it, it seems difficult to figure out exactly how Frege's remarks should be understood in the relevant context. As a matter of fact, he intersperses explanations only sparingly in $\$ 31$ (and elsewhere). Heck (1997, p. 467, footnote 8) suggests, without providing much explanation, that Frege cannot be talking here about the defined symbols to be introduced later in Part I of Grundgesetze: names of these functions are already present in the language. The latter claim seems to be true, but it does not justify the former. (i) Once all primitive function-names of the concept-script have been introduced and (ii) by stipulation none will be introduced at a later stage in the foundational project, and, furthermore, (iii) the formation rules are specified, we can indeed refer to any function and any object later to be defined in the concept-script. However, we must be careful here. Clearly, the one-place first-level function-names that Frege defines in later sections, such as the single-valuedness of a relation: "I $\xi$ " ( $\$ 37)$, the mapping-into by a relation: " $\rangle \xi$ " (\$38), the converse of a relation: " $\$ \xi "(\$ 39)$, etc. are at the stage of $\S 31$ not yet included in the concept-script. This consequently applies also to their corresponding valuerange terms " " $\varepsilon(\mathrm{I} \varepsilon)$ ", “' $\dot{\varepsilon}(\rangle \varepsilon)$ ", " $\varepsilon(\& \varepsilon)$ ", etc. Take, for example, "I $\xi$ ". Strictly speaking, we cannot even say that at the stage of $\S 31$ the definiens of "I $\xi \xi$ ", namely " $\forall x \forall y(x \cap(y \cap p) \rightarrow$ $\forall z(x \frown(z \frown p) \rightarrow y=z))$ " (I use here partly modern notation), is already present in the concept-script. The reason is that this function-name contains as a part the dyadic functionname " $\xi \cap \zeta$ " which is only later defined in $\S 34$. At the stage of $\S 31$, we can only say that a name of the single-valuedness of a relation is supposed to be included in the concept-script, if in " $\forall x \forall y(x \cap(y \cap p) \rightarrow \forall z(x \cap(z \cap p) \rightarrow y=z))$ " the name " $\xi \cap \zeta$ " is considered to be replaced by its definiens " $\backslash \dot{\alpha}(\neg \forall \mathfrak{g}(u=\dot{\varepsilon}(\mathfrak{g}(\varepsilon)) \rightarrow \neg \mathfrak{g}(a)=\alpha))$ ". And once " $\dot{\varepsilon} \varphi(\varepsilon)$ " has been introduced (according to Frege's statement, as late as in $\$ 31$ ), a value-range name that is coreferential with " $\dot{\varepsilon}(\mathrm{I} \varepsilon)$ " is likewise available in the concept-script. It is available in the sense that it can be constructed from the primitive names by iterated application of the formation rules.

The upshot is that we cannot safely rule out that in his initial remark on " $\dot{\varepsilon} \varphi(\varepsilon)$ " and the proper names associated with " $\dot{\varepsilon} \varphi(\varepsilon)$ " Frege is appealing to those value-range names which originate from the simple monadic first-level function-names later to be defined. Now, (i) if in the remark that I am discussing Frege does not tacitly refer to those simple function-names (= Heck's assumption), and (ii) if he holds, as he must, that at the stage of $\$ 31$ a complex name of any function later to be defined is already available in the concept-script, then it remains to ask which monadic first-level function-names he may have had in mind when he says that they may yet be introduced. 
The most plausible answer is presumably this. If the assumptions (i) and (ii) hold, then the monadic first-level function-names that may yet be introduced must be new primitive monadic function-names of first level as well as those monadic first-level function-names that can be obtained from the former and the old primitive names plus those already defined by applying the formation rules. When exposing the concept-script in $\S 1-\S 52$, Frege apparently did not rule out that at a later stage in the execution of his logicist programme - say, when laying the logical foundations of real analysis and of the arithmetic of complex numbers was on the agenda-it might have been useful or even mandatory to introduce a new primitive function-name or even more than one. By contrast, it seems very likely that he took the formation rules of his concept-script in Frege (1893) to be fixed once and for all after having characterized them in $\$ 26-\$ 28, \S 30$.

A final comment on Frege's statement in $\S 31$ that with " $\dot{\varepsilon} \varphi(\varepsilon)$ " we introduce not only a new function-name, but at the same time new proper names, is in order. Taken at face value, it is at variance with his previous exposition. Here are the reasons. In $\S 3$, he already introduces the metalinguistic counterpart of " $\varepsilon \varphi(\varepsilon)$ ". In $\S 10$, after having explained the notation for value-ranges in the preceding section, Frege appeals to the contextual stipulation in $\$ 3$ by employing the value-range notation. Furthermore, in $\$ 20$ he presents the formal version of Axiom V, in whose expression " $\varepsilon \varphi(\varepsilon)$ " figures as a constituent name. Finally, " $\dot{\varepsilon} \varphi(\varepsilon)$ " makes its first separate appearance in $\$ 22$ - "In $\dot{\varepsilon} \varphi(\varepsilon)$ we have an example of a second-level function which is not a concept"-and not in $\$ 31$, contrary to what Frege's statement in $\S 31$ regarding " $\varepsilon \varphi(\varepsilon)$ " suggests. Clearly, when he turns to " $\varepsilon \varphi(\varepsilon)$ " in $\S 31$, he cannot undo what he has done before and expect that the reader is prepared to disregard at least temporarily some of his previous stipulations in the spirit of "out of sight, out of mind". Note in this connection that a little later in $\S 31$ and in contrast to his previous claim that with " $\varepsilon \varphi(\varepsilon)$ " he introduces a new function-name, he invokes the twin stipulations in $\S 10$ - besides appealing to the stipulation in $\S 3$ in terms of the value-range notation and the first-order universal quantifier (as before in $\$ 10$ ) - by reformulating them in the semantic mode: 'Owing to our stipulations that. . ' ' $\varepsilon(-\varepsilon)$ ' is to refer to the True and that ' $\mathscr{\varepsilon}\left(\varepsilon=T^{\mathfrak{a}} \mathfrak{a}=\mathfrak{a}\right)$ ' is to refer to the False..." 35 In any event, Frege seems to believe that only by proceeding in the way he actually does in $\$ 31$ concerning the scope of the firstorder domain — recall that in $\$ 31$ he initially restricts it to the truth-values and subsequently extends it by including value-ranges — and his treatment of " $\dot{\varepsilon} \varphi(\varepsilon)$ " as a new, hitherto unknown function-name he is able to demonstrate convincingly that regular value-range names are indeed referential.

\section{\$7. Only in the context of a truth-value name (sentence) do value-range names} have a reference. To show that " $\mathfrak{a}-\varphi(\mathfrak{a})$ " has a reference requires only an application of criterion

$$
\text { (D): } \quad(\mathbb{a}-\mathfrak{a}-\varphi(\mathfrak{a}) \leftrightarrow \forall \Phi(\mathbb{B} \Phi(\xi) \rightarrow(\mathfrak{B})-\mathfrak{a}-\Phi(\mathfrak{a})) .
$$

The demonstration that " $\mathfrak{f}-\mu_{\beta}(\mathfrak{f}(\beta))$ " has a reference proceeds in a similar way. By contrast, the proof that " $\varepsilon \varphi(\varepsilon)$ " is referential requires, in addition to the application of criterion (D), an application of criteria (B) and (A).

35 It is clear that Frege's remark in $\$ 31$ concerning " $\varepsilon \varphi(\varepsilon)$ "- that with it we introduce not only a new function-name but at the same time new proper names-is not on a par with the introduction of value-ranges via an abstraction principle in $\S 3$ which lays down identity conditions for them, although in a restricted way. In my opinion, the remark in $\$ 31$ amounts roughly to the statement in $\$ 9$ that " $\varepsilon \Phi(\varepsilon)$ " refers to the value-range of $\Phi(\xi)$. 
According to (D), we have

(囚) $\dot{\varepsilon} \varphi(\varepsilon) \leftrightarrow \forall \Phi(\AA) \Phi(\xi) \rightarrow(\AA) \dot{\varepsilon} \Phi(\varepsilon))$.

As to the consequence, namely the target names for " $\dot{\varepsilon} \varphi(\varepsilon)$ ", criterion (B) applies

(B) $\dot{\varepsilon} \Phi(\varepsilon) \leftrightarrow \forall X(\mathbb{R} X(\xi) \rightarrow \mathbb{B} X(\dot{\varepsilon} \Phi(\varepsilon)))$

$\wedge \forall \Psi(\AA)(\xi, \zeta) \rightarrow \circledR \Psi(\dot{\varepsilon} \Phi(\varepsilon), \zeta) \wedge \AA \Psi(\xi, \grave{\varepsilon} \Phi(\varepsilon)))$.

And to " $\Psi(\grave{\varepsilon} \Phi(\varepsilon), \zeta)$ " and " $\Psi(\xi, \grave{\varepsilon} \Phi(\varepsilon))$ "—-both monadic function-names are target names for a canonical value-range name " $\dot{\varepsilon} \Phi(\varepsilon)$ "—criterion (A) applies

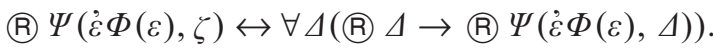

(®) $\Psi(\xi, \grave{\varepsilon} \Phi(\varepsilon)) \leftrightarrow \forall \Delta(\AA) \Delta \rightarrow$ ( $\Psi(\Delta, \grave{\varepsilon} \Phi(\varepsilon)))$.

In order to demonstrate that value-range names are referential, Frege must only consider those that are formed from referential monadic first-level function-names, that is, regular value-range names. Since "__ $\xi$ " and " $-\xi$ " had already been shown to be referential,

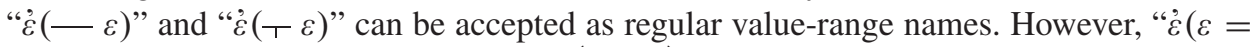

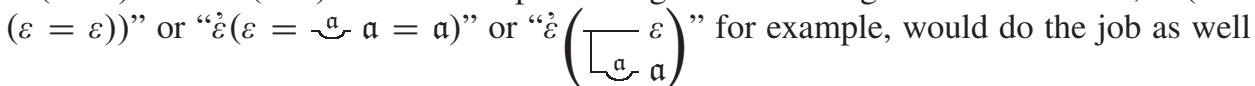
since the corresponding monadic function-names " $\xi=(\xi=\xi)$ ", " $\xi=\mathfrak{a}_{-} \mathfrak{a}=\mathfrak{a}$ " and “ ${ }_{\mathfrak{a}-\mathfrak{a}} \xi "$ are correctly formed from primitive names which, according to Frege, had already been established as referential in the first, simple part of the proof. By the first part, I mean here the demonstration of referentiality for the primitive concept- and relation-expressions of the formal language.

According to the criterion of referentiality (B), Frege must examine whether by inserting a regular value-range name into the argument-place of " $-\xi$ " and " $-\xi$ " we form a referential proper name, and further whether by inserting such a name into the $\xi$ - or $\zeta$ argument-place of " $\xi=\zeta$ " and " $\zeta$ ", we obtain a referential monadic function-name of $[\zeta$

first level. Thus, in the case of " $\varepsilon \varphi(\varepsilon)$ " Frege restricts the demonstration of referentiality to certain subsets of both the set of value-range names and the set of monadic first-level function-names of the concept-script, namely to the insertion of a regular value-range name into the argument-place(s) of the primitive monadic and dyadic first-level function-names. This procedure is almost the mirror image of the method that he proposes in $\$ 10$ for removing the referential indeterminacy of value-range terms. Recall that this method is restricted to the determination of the values of the primitive first-level functions for valueranges (and the truth-values) as arguments, although with no constraint imposed on the range of admissible value-ranges as arguments. Yet again, the investigation concerning " $\varepsilon \varphi(\varepsilon)$ " in $\S 31$ is essentially a semantic acid test, whereas the procedure in $\S 10-\S 12$ is intended to solve the indeterminacy problem by fixing the references of value-range names step by step up to uniqueness. And it takes little imagination to see that $\xi=\zeta$ plays a pivotal role when Frege comes to determine the values of the primitive first-level functions for value-ranges (and the truth-values) as arguments in $\$ 10$ and beyond. ${ }^{36}$

36 This applies independently of the fact that for the concept $-\xi$ the determination of its values for fitting arguments turns out to be unnecessary and that $-\xi$ is reducible to $\xi=\zeta$. Yet by determining (a) the truth-value of canonical value-range equations by reference to the contextual stipulation and (b) the truth-value of mixed equations of the form " $\varepsilon \Phi(\varepsilon)=\Delta$ " by additionally invoking the dual stipulation in $\S 10$, Frege thinks that he can guarantee precisely this: that the 
Guided by the piecemeal strategy launched in $\$ 10$ and bearing in mind the key role assigned to $\xi=\zeta$ in this strategy, Frege proceeds as follows. In the first place, the $\xi$ - or $\zeta$-argument-place of " $\xi=\zeta$ " is filled with a regular value-range term, say, with " $\dot{\varepsilon}(-\varepsilon)$ " or " $\dot{\varepsilon}(-\varepsilon)$ ". In order to show that, for example, " $\dot{\varepsilon}(-\varepsilon)=\zeta$ " is referential, we must examine, according to criterion (A), whether all proper names that we obtain by inserting either a truth-value name or a regular value-range name into the argument-place of " $\dot{\varepsilon}(-\varepsilon)=\zeta$ " are referential. Plainly, the proper names so obtained are truth-value names. In the light of Frege's initial assumption of propositional referentiality, we could therefore expect that they are recognized without further ado as referential names. Yet as we shall see below, Frege no longer appeals to this assumption when he comes to demonstrate that regular value-range names have a reference. This does of course not mean that he no longer relies on it. He rather wishes to establish the referentiality of, say, " $\dot{\varepsilon}(-\varepsilon)=\dot{\varepsilon}(\top \varepsilon)$ " or “a $-\mathfrak{a}=\dot{\varepsilon}(-\varepsilon)$ ” by expressly invoking the stipulations in $\S 3$ and $\S 10$.

It goes almost without saying that the truth-value name or the regular value-range name to be inserted into the argument-place of " $\dot{\varepsilon}(-\varepsilon)=\zeta$ " or " $\xi=\dot{\varepsilon}(-\varepsilon)$ " for example, must be a well-formed concept-script expression. Auxiliary proper names are excluded from the range of permissible substitution instances since they are not formed from the primitive function-names by applying any of the formation rules of the formal language. ${ }^{37}$ It is precisely this condition that every proper name must satisfy in any proof of

values of every other primitive first-level function for value-ranges as arguments are determined according to their elucidations. The twin stipulations in $\$ 10$ imply the reducibility of any equation of the form " $\dot{\varepsilon} \Phi(\varepsilon)=\Delta$ "- where " $\Delta$ " represents a truth-value name of the concept-script-to a canonical value-range equation. And after having carried out the reduction for any equation " $\dot{\varepsilon} \Phi(\varepsilon)=\Delta$ " we are in a position to determine its truth-value by appeal to the contextual stipulation. The special role that $\xi=\zeta$ plays in the piecemeal process of bestowing a unique reference upon value-range names can also be seen from a different angle. Suppose that in the logical system of Grundgesetze " $\xi=\zeta$ " was not treated as a primitive name, but was introduced via a definition modelled upon, say, Leibniz's definition of "=" which in Grundlagen Frege adopted as his own: $a=b:=\forall f(f(a) \leftrightarrow f(b))$. It is likewise plain that prior to the definitional introduction of " $\xi=\zeta$ " and prior to any possibly intended transsortal stipulations along the lines of those in $\S 10$ Frege would have been unable to fix the values of any primitive first-level function of his system for value-ranges as arguments.

37 On the one hand, Heck emphasizes correctly that according to Frege a name like " $\Delta$ " does not belong to the concept-script at all. On the other hand, he contends that it is a new primitive name, added to the concept-script (cf. Heck (1997), pp. 442, 448). First, there is no evidence that Frege construed " $\Delta$ " and other auxilary names, which he employs in the course of developing the concept-script, as new primitive names. Why should he do this? And what would be the justification for doing this once it is clear that such names do not belong to the concept-script? From Frege's point of view in Grundgesetze, only certain function-names that belong intrinsically to the language of first- and second-order logic as well as to the vocabulary of the theory of valueranges can be regarded as primitive or logically simple. (Note that the name of the horizontal function, which could be read as "the truth-value of $\xi$ 's being the True", plays a special role in the concept-script.) It is characteristic of the primitive names of the formal language (with the exception of "हे $\varphi(\varepsilon)$ ") that they are introduced by means of informal elucidations. Second, Frege does not hold the view that the concept-script is extended by adding auxiliary names. Since auxiliary names are strictly excluded from the range of admissible concept-script expressions, they cannot be added to it in any plausible sense. Heck writes: “. . it is implicit in Frege's argument that ' $\Phi(\Delta)$ ' denotes that ' $\Delta$ ' is not itself formed in the second way. But if ' $\Delta$ ' is an arbitrary denoting name in Begriffsschrift, there is no reason to suppose that it is not formed in the second way-as, indeed, it would be were it either ' $\dot{\varepsilon} \cdot \varepsilon=\varepsilon^{\prime}$ ' or ' $\forall x \cdot x=x$ ' -whence the syntactic claim simply does not apply, and the argument collapses" (p. 448). However, Heck thinks that the argument "goes through", if " $\Delta$ " is considered to be a new primitive name because 
referentiality for the concept-script that is governed by the criteria (A)-(E), regardless of whether the name functions as a test name or as a base name associated with it (according to the relevant criterion of referentiality) or as a target name. If in his attempted proof that every well-formed name of the concept-script has a unique reference Frege were to consider auxiliary proper names to be on a par with the primitive names and the names formed from these by way of insertion and gap formation, he would probably not succeed in proving anything at all. Admittedly, being neither primitive nor having been formed from the primitive function-names characterizes also every (simple) proper name introduced by definition, but unlike an auxiliary proper name, a defined proper name such as " 0 " or " 1 " or " $\aleph_{0}$ " is considered to belong to the concept-script. ${ }^{38}$ In contrast to the former, it has a well specified reference, namely the reference of a complex name that was correctly formed from the primitive names. It is true that in $\$ 31$ Frege employs the auxiliary names " $\Gamma$ " and " $\Delta$ " but-setting his vacillation between an objectual and a substitutional interpretation of first-order quantification aside-he does so chiefly for the purpose of simplifying his argument and of securing its requisite generality, not for any intrinsic reason. Strictly speaking, in $\$ 31$ " $\Gamma$ " and " $\Delta$ " are used to represent or stand for any truth-value name or any regular value-range name of the formal language. However, it does not make an awful lot of sense to say that " $\Gamma$ " and " $\Delta$ " are truth-value names or regular value-range names.

Having arrived at the point regarding " $\dot{\varepsilon} \varphi(\varepsilon)$ " that I described above, Frege appeals to both the contextual stipulation in $\$ 3$ and the identification of the True and the False with their unit classes in $\S 10$. As I pointed out earlier, on the face of it, this contravenes one of the preliminary remarks in $\$ 31$, namely that the only objects that we know at this stage are the True and the False. Be that as it may, Frege considers the stipulations in $\S 3$ and $\S 10$ to ensure that every name of the form " $\Gamma=\Delta$ " has a reference, if " $\Gamma$ " and " $\Delta$ " stand for regular value-range names or truth-value names. ${ }^{39}$ By the same token, it is clear that we always obtain a referential proper name, if we fill the argument-place of " $\xi=(\xi=\xi)$ " everywhere with a regular value-range name. ${ }^{40}$ The resulting names are equations (truth-value names), where the term occurring on the left-hand side is a regular value-range name and the name on the right-hand side is of the form " $\Delta=\Delta$ ", and, hence, refers to the True. Thanks to the dual stipulation in $\S 10$, the right-hand side

so considered it is clearly not formed in the second way. Again, in my judgement this is most likely not Frege's view of the matter. Moreover, it is possibly misleading to say, as Heck does, that " $\dot{\varepsilon}(\varepsilon=\varepsilon)$ " and " $\mathfrak{a}-\mathfrak{a}=\mathfrak{a}$ " are formed in the second way, that is, by means of gap formation. The last member of the formation sequences of these names is formation by insertion, not by gap formation. However, the construction of these names involves the antecedent application of a gap formation rule.

38 In Grundgesetze, " $\approx$ " is Frege's symbol for the smallest transfinite cardinal number. He calls this number Endlos; cf. Frege (1893), §122. Frege distinguishes typographically between the numerals " $Q$ " and " 1 ", that are supposed to refer to the cardinal numbers $Q$ and $\mathrm{A}$, and the numerals " 0 " and " 1 " that are supposed to refer to the numbers 0 and 1 (cf. Frege (1893), §41-\$42 and Frege (1903), $\S 157$, p. 156).

39 I assume that in this formulation Frege appeals only to truth-value names (sentences) of the concept-script, not to nonsentential names of a truth-value such as " $\varepsilon(-\varepsilon)$ " or " $\dot{\varepsilon}(\varepsilon=(\varepsilon=$ $\varepsilon)$ )", for example. We already know that " $\Gamma=\Delta$ " is not a name of the concept-script, although the constituent name " $\xi=\zeta$ " belongs to it (disregarding the preliminary use of " $\xi$ " and " $\zeta$ " in the exposition of the concept-script). " $\Gamma=\Delta$ " is an auxiliary name just as the name " $\Phi(\Delta)$ " which consists of the auxiliary names " $\Phi(\xi)$ " and " $\Delta$ ".

40 Frege speaks here of argument-places in the plural. This is not quite correct. " $\xi$ " marks only one argument-place in " $\xi=(\xi=\xi)$ ", though in different places. 
of an equation such as " $\dot{\varepsilon}(-\varepsilon)=(\grave{\varepsilon}(-\varepsilon)=\grave{\varepsilon}(-\varepsilon))$ " or " $\dot{\varepsilon}(-\varepsilon)=\left(\grave{\varepsilon}\left(\top^{-\varepsilon}\right)=\right.$ $\dot{\varepsilon}(-\varepsilon))$ " can always be replaced by " $\dot{\varepsilon}(-\varepsilon)$ ". If we do this, we obtain a canonical value-range equation whose truth-value is determined by the contextual stipulation or its formal counterpart Axiom V. In the first case we obtain a true, in the second a false equation. Due to the coextensiveness of $\xi=(\xi=\xi)$ and $-\xi$, “- $-\xi$ " is likewise always completed to a referential name, a truth-value name, if we insert a regular value-range term into its argument-place. And according to the stipulations made in $\S 5, \S 6, \S 12$, “- $\triangle$ " and " $\Gamma$ " are referential names, if " $\Gamma$ " and " $\Delta$ " stand for proper names of the concept-script that refer to something. Since regular value-range names satisfy this condition, both " $\top \xi$ " and " $\zeta$ " are always completed to referential proper names, namely truth-value names, $\zeta$

when we insert regular value-range names or truth-value names into the argument-places. Here then is the conclusion which Frege draws in $\$ 31$ after having completed his proof of referentiality:

We have seen that each of our names of simple first-level functions hith-

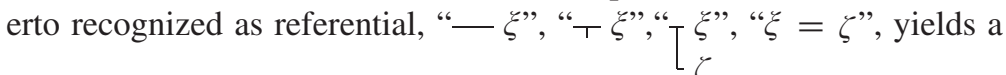
referential name if we insert regular value-range names into the argumentplaces. Thus, the regular value-range names may be included in the domain of referential names. Thereby, however, the same is settled for our function-name " $\dot{\varepsilon} \varphi(\varepsilon)$ ", since whenever a name of a first-level function with one argument refers to something, it follows that the proper name that results from its insertion into " $\dot{\varepsilon} \varphi(\varepsilon)$ " refers to something.

Finally, in $\S 31$ Frege argues that his stipulations in $\S 11$ suffice to secure a reference in all cases for proper names of the form " $\triangle$ " and consequently also for the primitive name "\} \xi \text { ". } 4 1

The foregoing analysis of Frege's argument in $\$ 31$ provides ample evidence that in his view value-range names and, hence, " $\dot{\varepsilon} \varphi(\varepsilon)$ " have a reference only in the context of a truthvalue name, of a sentence. His contextual account of both sense and reference for his formal language accords well with the context principle which assigns a distinguished role to sentences. Thus contrary to what Dummett has claimed in several places, in Grundgesetze Frege does not advocate a sheer generalization of the context principle with respect to reference. As Frege's statement at the beginning of $\$ 32$ makes clear, he believes to have shown (a) that all primitive names refer to something and (b) that all names correctly formed from these inherit their property of being referential. With respect to the class of value-range names this means that every canonical value-range name is assumed to have a (unique) reference, not only those names that were initially singled out as regular valuerange names.

This completes my account of Frege's proof of referentiality. In the last two sections of this essay, I shall return to Grundgesetze, $\S 10$ — bearing in mind that $\S 10$ is tightly interwoven with $\$ 31$ - and shall add a few more brushstrokes to the picture that I have painted so far of the semantics of value-range names. I shall begin by taking a closer look at Frege's piecemeal strategy in $§ 10$. In what follows, I critically examine his line of thought in the long footnote to $§ 10$. To my mind, this important footnote has not yet been

41 Cf. also Heck's analysis in Heck (2012), §5.1 and §5.3. 
discussed in sufficient detail in the relevant literature on $§ 10$. In the concluding section, I shall raise two objections to the twin stipulations in $\S 10$. The first is set out from the point of view of the syntax of Frege's formal language, while the second is based on semantic considerations.

\section{§8. Grundgesetze, §10.}

8.1. The piecemeal strategy. At the outset of Grundgesetze, $\S 10$, Frege points out that the contextual stipulation does not yet by any means fix the reference of a name such as " $₫ \Phi(\varepsilon)$ " completely. In what follows, he draws attention to a "dual undecidability". (U1): "we cannot decide yet [that is, by appeal to the contextual stipulation] whether an object that is not given to us as a value-range is a value-range or which function it may belong to". (U2): we cannot "decide in general whether a given value-range has a given property [that is, falls under a given first-level concept ${ }^{42}$ ] if we do not know that this property is connected with a [second-level ${ }^{43}$ ] property of the corresponding function." Note that both (U1) and (U2) are formulated in epistemic terms, while the initial statement in $\S 10$ is framed in semantic terms. As to the objects that are not given to us as value-ranges referred to in (U1), Frege deals in effect only with the two truth-values. However, when in the long footnote to $\$ 10$ he comes to explore the possibility of generalizing the transsortal stipulations, any arbitrary nonlogical object that is not referred to by a value-range name is tentatively taken into consideration as well. ${ }^{44}$ (U2) is closely intertwined with (U1) but does not coincide with (U1). We shall see that in a sense (U2) is a generalization of (U1). We shall further see that (a) by converting (U1) via the twin stipulations into a decidable issue and (b) by drawing on the elucidations of the primitive first-level function-names other than " $\xi=\zeta$ "- the name of the horizontal function, negation, the definite description operator and the name of the conditional function-we can decide in general whether a given value-range falls under a first-level concept (denoted by a concept-script functionname). ${ }^{45}$

After having stated (U1) and (U2), Frege presents an argument for his previous claim that the contextual stipulation fails to determine the reference of a name such as " $\dot{\varepsilon} \Phi(\varepsilon)$ " completely. (Here I skip the argument. ${ }^{46}$ ) He then proceeds to characterize the piece-

42 In (Frege, 1892, p. 175), Frege writes: "I call the concepts under which an object falls its properties; thus 'to be $\Phi$ is a property of $\Gamma$ ' is just another way of saying: ' $\Gamma$ falls under the concept of a $\Phi$ '.'

43 The property must be of second level since the function corresponding to the value-range is of first level. Recall that in Grundgesetze Frege introduces and uses only value-ranges of first-level functions. And plainly, in his logic a first-level function can only have a second-level property.

44 To be sure, Frege introduces only the truth-values and value-ranges as logical objects and nowhere in his work mentions any other logical objects or the need to extend the domain of logical objects beyond the truth-values and value-ranges. Yet this does not exclude that in the course of laying the logical foundations of real analysis (which due to Russell's Paradox remained a fragment in the second volume of Grundgesetze) and the arithmetic of the complex numbers (possibly in a third Grundgesetze volume) he would have been prepared to introduce logical objects of a new third kind. I shall return to this idea shortly.

45 In saying this, I do not consider the if-clause in (U2) which Frege does not spell out in §10. I do this mainly for the sake of simplifying my account. Yet, as we shall see, the determination of the reference of, say, "לे $(-\varepsilon)=\mathfrak{a}-\mathfrak{a}$ " enables Frege to fix the reference of, say, " $T \grave{\varepsilon}(-\varepsilon)$ " as well.

46 Actually, he states that this claim follows from his argument. I assume that the informed reader is familiar with the two arguments that Frege develops in $§ 10$. 
meal strategy as the appropriate means of resolving the indeterminacy that he diagnoses at the outset of $\S 10$. At the stage of $\S 10$, the proposed solution, namely the step-bystep determination of the values of the primitive first-level functions for value-ranges as arguments, "just as for all other arguments", eventually boils down to the case of $\xi=\zeta$, and "all other arguments" are reduced to the truth-values, although not for a compelling reason. If we take Frege's proposal at face value, then there are altogether three cases to be settled for $\xi=\zeta$. However, he confines himself to considering the case where one argument of $\xi=\zeta$ is a value-range and the other a truth-value which in essence corresponds to (U1). One of the two remaining cases, the determination of the values of $\xi=\zeta$ for value-ranges as $\xi$-argument and as $\zeta$-argument, was already done in $\S 3$ and, hence, Frege did not have to worry about it. It is probably for this reason that he does not mention this case at all when he presents his solution strategy in response to (U1) and (U2). Yet he does rely on this case since it is absolutely indispensable for fixing the semantics of value-range names, although at the same time it gives rise to the indeterminacy that is in the focus of $\S 10$. Frege likewise disregards the third case (at least he does not mention it explicitly), which is the determination of the value of $\xi=\zeta$ for the True and the False as $\xi$-argument and as $\zeta$-argument. It is possible that he tacitly appeals to his previous elucidation of " $\xi=\zeta$ " (which on its own does not decide the third case) and assumes that thanks to our unfailing awareness, in our practice of judging and asserting, that the True is distinct from the False, ${ }^{47}$ fixing the value of $\xi=\zeta$ for the truth-values as arguments can be taken for granted. However this may be, Frege possibly thought that from the third case he could hardly derive much benefit for specifying the semantics of value-range names, and it is perhaps also for this reason that he omits it.

Having arrived at this point in $§ 10$, Frege sets out the permutation argument and goes on to state the identifiability thesis. By invoking the latter, he identifies the True and the False with their unit classes. ${ }^{48}$ Thanks to this dual transsortal identification, the intended

47 In 'Über Sinn und Bedeutung' (Frege, 1967, p. 149; cf. Frege, 1893, p. 7), Frege claims that the two objects the True and the False are acknowledged, if only tacitly, by everybody who judges at all, who holds something to be true and, hence, also by a sceptic.

48 In their essay 'Frege's Recipe', Cook and Ebert write (Cook \& Ebert, 2016, p. 321): "Given that in Grundgesetze sentences are names of truth-values, the logic of Grundgesetze involves, at a glance, reference to two distinct types of logical object: truth-values and value-ranges. In order to reduce the number of types of logical objects-with a view to settling all identities within Grundgesetze in terms of identity conditions for value-ranges as codified in Basic Law V-Frege makes two stipulations." It could seem that the authors are assuming here that reducing the number of types of logical object was a desideratum for Frege even independently of the fact that the referential indeterminacy of value-range names was in urgent need of being removed. Be that as it may, in the light of the available evidence, Frege's conversion of the True and the False into objects whose identity is governed by the coextensiveness of certain concepts is in fact only due to the indeterminacy problem and his attempt to resolve it. In my judgement, he did not think that for methodological reasons and quite independently of the semantic problem that was preoccupying him in $\$ 10$ he was committed to requiring that the identity conditions of every logical object of his system must or should be governed by a logical abstraction principle, preferably by Axiom V. At least prior to $\S 10$, the True and the False are considered to be the primitive objects of logic. Unlike the value-ranges, the truth-values do not depend on something more fundamental, namely on functions. It is true that the value-range function $\dot{\varepsilon} \varphi(\varepsilon)$ is primitive in Frege's system, but this status is not transmitted to its values for monadic first-level functions as arguments: the (simple) value-ranges. Thus, in a sense one could say that the truth-values are initially in the domain of logical objects what the primitive functions of the system of Grundgesetze are in the domain of functions; see in this respect the revealing passage in Frege (1976), p. 121 and the comments in Schirn (2006a, 2018b). Moreover, I take for certain that Frege did not believe that by identifying 
complete determination of the references of value-range names is now supposed to come within reach. Nevertheless, at the end of $\S 10$ Frege points out that a little more work has still to be done in subsequent sections $(\$ 11-\S 12)$ in order to achieve this goal.

In summary, we can say that the piecemeal process of fixing the references of valuerange names uniquely is a sequence of heterogeneous stipulations which are designed to work hand in hand. The sequence itself falls neither under the prohibition of piecemeal definitions nor under the prohibition of piecemeal elucidations (of primitive names). Its first member is a stipulation of a special kind, while the second is a stipulation of a "very special kind", to borrow a phrase from Frege which he uses in a different context. Both (a) the contextual stipulation in $\S 3$ and (b) the twin stipulations in $\S 10$ are neither definitions nor standard elucidations. In the sequence, (a) and (b) are followed by (c) the elucidation of the definite description operator in $\$ 11$ and (d) the elucidation of the name of the conditional function in $\$ 12$. Yet both elucidations do their job of bestowing a reference on a new primitive name at one fell swoop and not in a piecemeal fashion. ${ }^{49}$

I shall now turn in slightly more detail to what from a semantic point of view I take to be the crucial issues involved in the piecemeal process that I characterized above. In doing so, I shall refrain from discussing the structure of the permutation argument. ${ }^{50}$ In Frege's view - at least I take this to be his view — once the value-range notation has been introduced in $\S 9$, the contextual stipulation in $\S 3$ can be seen as having conferred references on valuerange names as constituents of canonical value-range equations " $\stackrel{\varepsilon}{\Phi} \Phi(\varepsilon)=\dot{\alpha} \Psi(\alpha)$ " by fixing the reference of such and only of such equations. No more and no less is required in order to provide " $\dot{\varepsilon} \Phi(\varepsilon)$ " and " $\dot{\alpha} \Psi(\alpha)$ " qua constituents of " $\dot{\varepsilon} \Phi(\varepsilon)=\dot{\alpha} \Psi(\alpha)$ " with a reference. In saying this, I assume, for the sake of argument, that at the stage of $\S 9$ the formation of concept-script function-names and object names via insertion (not yet via gap formation) has already been put into effect. Thus, on this assumption it seems that at the stage of $\$ 9$ Frege would be justified, from his point of view, in writing down conceptscript sentences such as " $-\grave{\varepsilon}(\varepsilon=7 \mathfrak{a}-\mathfrak{a})=\grave{\varepsilon}(-\varepsilon)$ " or " $-\grave{\varepsilon}(-\varepsilon)=\dot{\varepsilon}(\varepsilon=(\mathfrak{a}-\mathfrak{a}=$

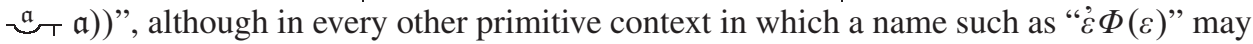
occur at this point—say, in "—— $\dot{\varepsilon} \Phi(\varepsilon)$ " or in " $-\bar{\varepsilon} \Phi(\varepsilon)$ " or in a mixed equation of the type " $\dot{\varepsilon} \Phi(\varepsilon)=\Delta$ ", where the auxiliary name " $\Delta$ " is supposed to represent a truth-value name of the concept-script ${ }^{51}$ — the reference of " $\varepsilon \Phi(\varepsilon)$ " "is by no means yet completely fixed" via

the truth-values with value-ranges he had, as a kind of welcome by-product, enhanced the logical status of the former. In fact, I do not see how this could have been achieved at all from his point of view. On the contrary, owing to the twin stipulations in $\S 10$, the truth-values had lost their pre-eminent status as the primitive objects of logic. Since they were now value-ranges of certain functions they had then only a derivative status.

49 Heck (2005, p. 168, footnote 17) writes that it is plain that Frege is not here offering a solution to the Caesar problem. "A piecemeal 'solution' is not a solution to the problem but a recipe for side-stepping it." However, Heck does not explain in more detail why he thinks that a piecemeal solution is not a solution to Frege's semantic problem. Admittedly, due to Frege's wholesale rejection of piecemeal definitions in logic and mathematics, the word "piecemeal" has a negative flavour in his mature work. Yet as I pointed out, the piecemeal process in $\$ 10-\S 12$ (with a tacit appeal to §3) does not concern any definition, nor does it rest on a piecemeal elucidation of a primitive first-level function-name. Thus, in my view Frege's piecemeal strategy does not offend against any of the methodological principles that guide his exposition of the concept-script.

50 I assume that the informed reader is familiar with Frege's permutation argument.

51 Here I apply the term "mixed equation" only to those equations in which one term flanking "=" is a canonical value-range name and the other a concept-script truth-value name. Beginning with $\S 11$, Frege introduces new types of singular terms (proper names in his jargon) into his formal language, both by elucidation and definition, such as definite descriptions of the form "\} \hat { \varepsilon } \Phi ( \varepsilon ) \text { " } 
the contextual stipulation together with the elucidations of " $-\xi ", " \leftarrow \xi$ " and " $\xi=\zeta$ " in $\S 5-\$ 7$. The reason is that "we cannot decide", by appeal to this set of stipulations, whether, for example, $\grave{\varepsilon} \Phi(\varepsilon)$ falls under $-\xi$ or under $+\xi$ or under $\xi=\mathfrak{a}-\mathfrak{a}$ or under none of these concepts. This is why I suggested construing (U2) as a generalization of (U1)..$^{52}$ The only case in which we can decide, by appeal to the contextual stipulation and prior to $\S 10$, whether, say, $\grave{\varepsilon} \Phi(\varepsilon)$ falls under a given first-level concept denoted by a conceptscript function-name is, for example, $\xi=\dot{\varepsilon}(-\varepsilon)$. Thus, it seems that at the stage of $\S 3-\S 9$ value-range names have a Janus-faced nature with respect to reference, or to put it in less metaphorical terms: From the point of view of the solution strategy which Frege proposes in $\$ 10$, the answer to the question of whether a (determinate) reference has been conferred on a name such as " $\stackrel{\varepsilon}{\varepsilon} \Phi(\varepsilon)$ " seems to be essentially context-dependent or context-relative. ${ }^{53}$

To recap: Thanks to the contextual stipulation-which despite its bounded effectiveness is a kind of bedrock for the semantics of value-range names ${ }^{54}$ - and prior to the stipulation in $\S 10$, a name such as " $\& \Phi(\varepsilon)$ " has a (determinate) reference if it occurs in " $\dot{\varepsilon} \Phi(\varepsilon)=$ $\dot{\alpha} \Psi(\alpha)$ ". If that were not so, then the equation " $\dot{\varepsilon} \Phi(\varepsilon)=\dot{\alpha} \Psi(\alpha)$ " would lack a reference, contrary to the fact that the contextual stipulation does fix its reference, that is, its truthvalue. By contrast, at the stage of $\S 9$ " $\& \Phi(\varepsilon)$ " lacks a (determinate) reference if it occurs in “—_ $\grave{\varepsilon} \Phi(\varepsilon)$ " or in " $T \dot{\varepsilon} \Phi(\varepsilon)$ " or in " $\dot{\varepsilon} \Phi(\varepsilon)=\Delta$ " since so far no stipulation has been made which fixes the reference of those sentences (truth-value names). As expected, for Frege the situation changes completely with the twin stipulations in $§ 10$. Casually speaking, we might say that, in his view, fixing the reference of mixed equations brings about an essential increase in the "referential potential" or "semantic value" of value-range names which initially rested only on the contextual stipulation. ${ }^{55}$ In fact, fixing the reference of a name such as " $\varepsilon \Phi(\varepsilon)$ " uniquely, that is, according to the piecemeal strategy, fixing its reference

and numerical terms of the form " $\not \dot{\varepsilon} \Phi(\varepsilon)$ " ("the cardinal number that belongs to the value-range $\grave{\varepsilon} \Phi(\varepsilon) ")$.

52 Note that my way of putting this deviates from Frege's formulation at the very outset of $\S 10$, but I think that my formulation, which takes Frege's subsequent statements into consideration, may render the issue a little clearer.

53 On the face of it, there seems to be a kind of weak analogy between the proposed piecemeal strategy in $\$ 10$ and a requirement that Frege states in Grundlagen when he comes to propose a contextual definition of the direction operator " $D(x)$ ". The requirement is that one must go beyond this tentative definition - and probably beyond that of the cardinality operator " $N_{x} \varphi(x)$ " as well — by adding further explanations or definitions in order to fix its reference uniquely. Thus, from Frege's point of view in Grundlagen, the tentative contextual definition of " $D(x)$ ", taken by itself, would fail to fix the reference of " $D(x)$ " uniquely even if the England problem concerning directions ("Does England coincide with the direction of the Earth's axis?") could be solved by appealing to an additional stipulation that were consistent with the contextual definition and did not rest on intuition or experience. Even in that case Frege would, I believe, concede that the definition effects only a partial determination of the reference of " $D(x)$ ". The reason is that his demand of supplying explanations or definitions of all other relevant statements about directions (besides identity statements) in order to fix the reference of " $D(x)$ " is set up quite independently of the impact that the England problem had on the acceptability of the contextual definition of " $D(x)$ " and the necessity of pursuing a new definitional strategy. In fact, Frege formulates the demand already in $\$ 65$ just before he sets up the contextual definition of " $D(x)$ ". As is well known, in Grundgesetze he rejects out of hand both contextual and piecemeal definitions.

54 Its undisputed merit is that it provides "a way always to recognize a value-range as the same if it is designated by a name such as ' $\check{\varepsilon} \Phi(\varepsilon)$ '”, a merit Frege could build on.

55 Note that the twofold stipulation in $\$ 10$ has no impact whatsoever on the references of valuerange names qua constituents of a canonical value-range equation since in a context of this type the value-range names flanking "=" on both sides already refer to something. They do so quite 


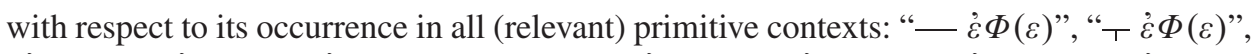

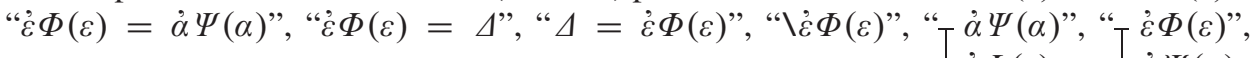
“ $\left[\begin{array}{l}\Delta " \\ \dot{\varepsilon} \Phi(\varepsilon)\end{array}\right.$ and " $\left[\begin{array}{c}\dot{\varepsilon} \Phi(\varepsilon) \text { " is nearly complete in } \S 10 .{ }^{56} \\ \Delta\end{array}\right.$

Thus, one result is that for Frege both canonical value-range equations and mixed equations are arguably the two most important primitive (sentential) contexts for the stepwise endowment of a name such as " $\dot{\varepsilon} \Phi(\varepsilon)$ " with a unique reference. ${ }^{57}$ Conferring a reference on " $\varepsilon \Phi(\varepsilon)$ " in these "identity contexts" ensures that in the light of the explanations of

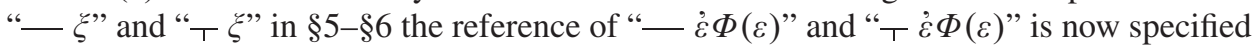
as well, not only the reference of " $-\Delta$ " and " $T \Delta$ " (where " $\Delta$ " represents again a truth-value name of the concept-script), as was initially the case when Frege provided elucidations of " - $\xi$ " and " $-\xi$ ". ${ }^{58}$ This only confirms what I have said at the outset of 8.1 , namely that by solving (U1) — which is clearly the central case when we are bound to decide whether a given value-range falls under a concept-Frege succeeds in solving (U2) as well. And recall that (U2), properly understood, is just a generalization of (U1). ${ }^{59}$

independently of any subsequent stipulation concerning their reference as constituents in other primitive contexts.

56 According to Frege, the unfinished semantic business left by the contextual stipulation in $\$ 3$ is officially completed by the stipulations that he makes in $\$ 11-\S 12$ regarding the definite description function and the conditional function both of which, unlike $-\xi$, are not reducible to any other primitive function. But according to the elucidations of " $\zeta$ " and " $\left[\begin{array}{l}\xi \text { " and the previous } \\ \zeta\end{array}\right.$ stipulations, the values of $\backslash \xi$ and $T_{\zeta}^{\xi}$ are also specified for value-ranges as arguments.

57 As I have indicated before, once the dual stipulation in $\$ 10$ has been made, mixed equations can of course always be transformed into canonical value-range equations, and the truth-value of the latter is determined via the contextual stipulation. It likewise goes without saying that owing to the twin stipulations in $\S 10$ a truth-value name can always be replaced salva extensione either by " $\varepsilon(-\varepsilon)$ " (or by a coreferential value-range term) or by " $\varepsilon(\varepsilon=, \mathfrak{a}-\mathfrak{a}=\mathfrak{a})$ " (or by a coreferential value-range term) but never salva intensione, since despite the fact that by stipulation it refers to a truth-value it expresses intrinsically a nonpropositional sense, not a thought. The key role of canonical value-range equations and mixed equations in the piecemeal process of endowing value-range names with a unique reference by no means implies that such equations are equally important in the proofs of the basis laws of arithmetic in the two volumes of Grundgesetze. (Cf. Frege's explanation of what he understands by an equation in the concept-script in Frege (1893), \$26.) As a matter of fact, the use of canonical value-range equations and especially of mixed equations has absolute scarcity value in the Grundgesetze-proofs, and from this we may conclude that those equations, in particular the mixed ones, play an extremely minor role in Frege's logical foundation of arithmetic. In $\S 177$, he uses " $\dot{\varepsilon} g(\varepsilon)=\dot{\varepsilon}(-f(\varepsilon))$ " as well as the mixed equations " $\varepsilon g(\varepsilon)=u$ " and " $\dot{\varepsilon}(-f(\varepsilon))=u$ " (where " $u$ " is a Roman object-marker). There are only a very few further occurrences of a canonical value-range equation. There is one in the course of the proof of the right-to-left-half of Hume's Principle in §61, p. 81 and also one in $\$ 88$, p. 116 , at the outset of the proof of the single-valuedness of "the relation of a cardinal number to that immediately preceding it in the cardinal number series". As far as I can see, this is all. In the proofs of Grundgesetze, Frege also draws very sporadically on equations in which the terms flanking "=" on both sides refer to double value-ranges; cf. $\$ 61$ and $\S 87$, p. 110 . Yet outside of equations it is the use of names for double-value ranges, not the use of names for simple value-ranges, that prevails in the proofs of Grundgesetze.

58 Recall that "concrete" truth-value names are not yet available at the stage of §5-\$6.

59 Heck shares this view; see Heck (2012), p. 104 and also the entire $\$ 4.4$ where in my judgement the relevant semantic issues of $\S 10$ are more thoroughly discussed than in any other work on Grundgesetze, $\S 10$. 
8.2. A closer examination of the idea of generalizing the dual stipulation in $\$ 10$. In a lengthy footnote to Frege (1893), §10, Frege examines the possibility of generalizing the dual stipulation (or " $\dot{\varepsilon}(\varepsilon=\Delta)=\Delta$ ") so that all objects whatsoever, including those that are referred to by canonical value-range names, are identified with their unit classes: $\forall \Delta \forall \dot{\varepsilon} \Phi(\varepsilon)(\dot{\varepsilon}(\varepsilon=\Delta)=\Delta \wedge \dot{\varepsilon}(\dot{\varepsilon} \Phi(\varepsilon)=\varepsilon)=\dot{\varepsilon} \Phi(\varepsilon)) .{ }^{60}$ On the face of it, the examination would not make an awful lot of sense if Frege had decided outright that the first-order domain of his logical theory is to embrace only the truth-values and value-ranges. In any event, he rejects the tentative generalization on the ground that it may be inconsistent with the criterion of identity governing value-ranges if the object to be identified with its unit class is already given to us as a value-range. At the same time, he jettisons the less general, but intuitively more appealing proposal to identify with their unit classes all and only those objects which are given to us independently of value-ranges: $\forall \Delta(\dot{\varepsilon}(\varepsilon=\Delta)=\Delta)$. He does so by using basically the same argument that he advances in Grundlagen, §67, saying that the mode of presentation or designation of an object must not be regarded as its invariant property, since the same object may be given in different ways. In short, in the footnote Frege tentatively considers both a restricted and a total generalization of " $\dot{\varepsilon}(\varepsilon=\Delta)=\Delta$ " but eventually rejects both as unviable, although for fundamentally different reasons. Yet assuming that the first-order domain is all-embracing, how could Frege believe he had an effective strategy for resolving the referential indeterminacy of value-range names if he is convinced that both a total and a restrained generalization of the dual stipulation in $\S 10$ are bound to fail?

In what follows, I shall discuss the relevant points that are more or less directly involved in the footnote. However, I shall disregard the shorter passage at its end since what Frege writes there is not my main concern.

(i) To begin with, Frege does not say anything about his motive for exploring the possibility of generalizing the transsortal stipulations in either way. We are only told that the identification of every object $\Delta$ with $\dot{\varepsilon}(\varepsilon=\Delta)$ suggests itself. But in what sense does it suggest itself? How does it relate to the indeterminacy problem whose solution is the key issue in $\S 10$ ? In particular, what would we achieve for the semantics of value-range names by generalizing the stipulation " $\Delta=\dot{\varepsilon}(\varepsilon=\Delta)$ " if at least the bounded generalization of " $\Delta=\dot{\varepsilon}(\varepsilon=\Delta)$ " were considered to be viable?

(ii) If (a) on the one hand we pay attention to the way Frege actually proceeds in $§ 10$ and especially in $\$ 31$, it is tempting to assume that he (tacitly) regards the first-order domain of his logical theory as restricted to the truth-values and value-ranges (cf. $\$ 2$ of this essay). Exploring the possibility of generalizing the transsortal stipulations in $\$ 10$ could then perhaps be regarded as an attempt to supply a general solution to versions of the indeterminacy problem that would inevitably arise for every possible extension of the first-order domain in the process of laying the logical foundations of real analysis and the arithmetic of the complex numbers. However, if (b) on the other hand Frege takes the firstorder variables to range over all the objects there are, then the second footnote to $\$ 10 \mathrm{might}$ rather be seen as reflecting his residual uneasiness about the restriction that he imposes on the range of admissible arguments when in $\S 10$ he determines the values of $\xi=\zeta$ only for the truth-values and value-ranges as arguments.

Both (a) and (b) deserve close attention. I shall begin by discussing a case in point that relates to (a). Suppose that Frege's projected logical foundation of the arithmetic of

60 I use here " $\Delta$ " as a variable ranging over singular terms distinct from every canonical value-range name. 
the real and complex numbers required the introduction of a new, third type of logical objects, besides the truth-values and value-ranges, and, correspondingly, the introduction of a new syntactic subcategory of singular terms that are designed to refer to those objects in the concept-script. In that case, too, the referential indeterminacy of value-range names that Frege apparently takes to be resolved at the end of $\S 12$, if only truth-values and valueranges are in the domain, would arise anew. An extension of what I hypothetically consider to be the first-order domain of Frege's logical theory could be imagined to proceed as follows: In addition to $\mathfrak{a}-\varphi(\mathfrak{a})$ and $\dot{\varepsilon} \varphi(\varepsilon)$, a third primitive monadic function of second level, say, $\hat{u} \varphi(u)$, is introduced via the following stipulation: its value for every one-place first-level function as argument shall be an object of the new third kind. I am aware that Frege might have been reluctant to endorse this way of introducing a new function $\hat{u} \varphi(u)$ on the ground that it illicitly presupposes our acquaintance with the values of $\hat{u} \varphi(u)$ qua logical objects of a third kind for monadic first-level functions as arguments. Nevertheless, let us assume, again for the sake of argument, that he would have accepted this way of introducing a function $\hat{u} \varphi(u)$, had he thought that its introduction was indispensable in pursuit of his overall logicist project.

From what I have just envisioned to be an extension of the initial, hypothetically assumed first-order domain, it would follow that the intended complete determination of the references of value-range names could not be secured. The reason is that from Frege's point of view we could not rule out that an object of the hypothetical third kind, which $\hat{u} \varphi(u)$ assigns to a fitting argument, does in fact coincide with a value-range in the domain. In order to remove this iterated indeterminacy, another stipulation would have to be made, one that it is designed to guarantee that the reference of every equation in which "=" is flanked by a canonical value-range name on one side and a singular term of the new category on the other is fixed. And, of course, every additional extension of the first-order domain in a similar fashion would require further stipulation.

In a letter to Russell of 3.8.1902, Frege makes a couple of interesting remarks which in a sense are closely linked to what I have just set out (Frege, 1976, p. 225):

You ask how it can be known that something is a value-range. This is indeed a difficult point. Now, all objects of arithmetic are introduced as value-ranges. Whenever a new object to be considered is not introduced as a value-range, we must at once answer the question whether it is a value-range, and the answer is probably always no, since it would have been introduced as a value-range if it was one.

Note that according to Frege a negative answer to the question that he raises would not be definitive, but only probable. Yet it seems that his response does not completely tally with the argument that he adduces in Grundlagen, $\$ 67$ and endorses in the long footnote to Grundgesetze, $\S 10$. In the light of this argument, we could never rule out that the new object to be considered (for laying the logical foundations of arithmetic) is a value-range, by invoking the fact that it has not been introduced as such, but, for example, as the reference of a name of the form " $\hat{u} \varphi(u)$ ".

I shall now turn to (b). Examining the possibility of identifying all and only those objects of an all-inclusive domain with their unit classes that are given to us independently of value-ranges might have assuaged the discomfort that Frege possibly felt at one point in $\S 10$. Recall that in contrast to his previous elucidations of some primitive first-level functions for all the objects there are he decides in $\S 10$, before fixing the values of those functions "for value-ranges as arguments, just as for all other arguments", to narrow down 
the range of arguments (and likewise the range of arguments for those primitive first-level functions that must still be introduced in the wake of $\S 10$ ) to the truth-values and valueranges. Testing a restricted generalization of " $\varepsilon(\varepsilon=\Delta)=\Delta$ " might appear attractive. For if every object that is not presented to us in the guise of a value-range were identified with its unit class, then it seems that we might succeed in removing effectively the referential indeterminacy of value-range names even if - in accordance with the way Frege elucidates the primitive first-level function-names and defines the names of certain logically complex functions of first level-the first-order domain is taken to be all-encompassing. Owing to the generalized stipulation " $\forall \Delta(\dot{\varepsilon}(\varepsilon=\Delta)=\Delta)$ ", the domain would contain only objects whose identity conditions are governed by the contextual stipulation in $\S 3$, and from Frege's point of view no further stipulation after $\$ 10-\$ 12$ would probably be required in order to ensure that value-range terms have been provided with a (unique) reference. Thus, if we are facing the question of whether, say, $\grave{\varepsilon}(\varepsilon=\varepsilon)$ is identical with Julius Caesar, we could, in principle, transform it into the question of whether $\dot{\varepsilon}(\varepsilon=\varepsilon)$ coincides with $\dot{\varepsilon}(\varepsilon=$ Julius Caesar $) .{ }^{61}$ And the criterion of identity inherent in the contextual stipulation would settle this question: $\xi=\xi$ and $\xi=$ Julius Caesar are obviously not coextensive. Hence, " $\dot{\varepsilon}(\varepsilon=\varepsilon)=\grave{\varepsilon}(\varepsilon=$ Julius Caesar)" is false. However, in special cases the determination of the truth-value of an equation in which both terms flanking "=" refer to unit classes, may turn out to be less straightforward. Thus, if we wish to decide, by invoking the contextual stipulation, whether, say, $\dot{\varepsilon}(-\varepsilon)$ is identical with $\dot{\varepsilon}(\varepsilon=$ Julius Caesar), we must be in a position to decide whether the concepts $-\xi$ and $\xi=$ Julius Caesar are coextensive or not and, hence, whether the True or $\grave{\varepsilon}(-\varepsilon)$ coincides with Julius Caesar or not. The question cannot be settled by any of the stipulations that Frege makes in Grundgesetze. Nonetheless, he might wish to argue that it is by virtue of our intuitive familiarity with the True and the False that we can safely distinguish the truth-values from the conqueror of Gaul and, hence, are entitled to declare " $\stackrel{\varepsilon}{(\varepsilon}=$ Julius Caesar $)=\dot{\varepsilon}(-\varepsilon)$ " as false. ${ }^{62}$

61 Here I ignore the fact that the concept-script does not contain names like "Julius Caesar". Note that Frege does not mention the fact that the concept-script does not contain names for all the objects there are.

62 The number 1 is obviously not given as a value-range by "1" and, according to the initial argument in the second footnote to $\$ 10$, could be identified with its unit class. However, since Frege intends to define individual cardinal numbers as equivalence classes of equinumerosity-for example, 1 as the class of all classes equinumerous with $\dot{\varepsilon}(\varepsilon=0)$, that is, as $\dot{\varepsilon}(\varepsilon \sim \dot{\varepsilon}(\varepsilon=0))$, if we use here " $\sim$ " as the sign for the relation of equinumerosity between classes- - he could not at the same time identify 1 with $\grave{\varepsilon}(\varepsilon=1)$ (its unit class) without contradicting the contextual stipulation or Basic Law V. Plainly, the concepts $\xi=1$ and $\xi \sim \hat{\varepsilon}(\varepsilon=0)$ are not coextensive. In recent correspondence (4 May 2017), Richard Heck endorses this argument and extends it: "Given how Frege defines ' 1 ', we certainly cannot have $1=\hat{x}(x=1)$, for essentially the reason Frege gives in the long footnote. Of course, the argument extends to other numbers. It's easy to show that if a given number is neither zero nor antizero (the number of $x=x$ ), then there are at least two concepts with that number: Let $F$ be some concept with the number in question; since the number is not zero, there is an $F$, say $a$; since the number is not antizero, there is a non- $F$, say $b$; now just swap $a$ and $b$, i. e., consider: $(F \xi \& \xi \neq a) \vee \xi=b$. That is equinumerous to and different from $F$. For the case of 0 , the argument is a bit more involved, since $0=\hat{x}(x \sim \hat{y}(y \neq y))=\hat{x}(x=$ $\hat{y}(y \neq y))$, so there is only one such $x$. But if $0=\hat{x}(x=0)$, then $\hat{x}(x=0)=\hat{x}(x=\hat{y}(y \neq y))$, so $0=\hat{y}(y \neq y)$, but then $\hat{x}(x \sim \hat{y}(y \neq y))=\hat{y}(y \neq y)$, which fails, since the former is not empty. Finally, antizero cannot be its own unit class, either, but that depends upon the fact that there are Dedekind infinitely many objects. But that is actually quite easy to prove. Just consider the sequence:

$$
\hat{y}(y \neq y)
$$




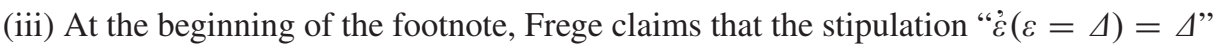
"is possible for every object that is given to us independently of value-ranges, for the same reason [my emphasis] that we have seen for truth-values." Frege does not explain what he means precisely when he says "for the same reason". I assume, however, that by using this phrase he is tacitly appealing to the permutation argument in the sense that its legitimizing power for the identification of objects with their unit classes is by no means restricted to the truth-values but could in principle be invoked for the identification of, say, Caesar and Brutus or Mars and Jupiter with their unit classes, if under the assumption that the firstorder domain embraces all objects whatsoever such a generalization were considered to be a viable strategy for fixing the references of value-range names uniquely. Surprisingly and in contrast to Frege's initial claim quoted above, his subsequent argument seems to be that it is consistent (a) to dismiss as indefensible the tentative proposal to identify with their unit classes all and only those objects that are not given to us as value-ranges and yet (b) to allow certain particular identifications which this proposal, if accepted, would also license. I cannot offer a catch-all-solution to this conflict, but I think that Frege would have been well-advised to stick to his initial claim.

If the consistency with the contextual stipulation is the only relevant constraint to be met if we ponder over the possibility of identifying every object that is not given to us in the guise of a value-range with its unit class - and thanks to the permutation argument Frege seems to be convinced that his twin stipulations in $\$ 10$ meet this constraint-then it could seem that nothing stands in the way of generalizing the stipulation " $\varepsilon(\varepsilon=\Delta)=\Delta$ " (for whatever purpose) in precisely this way. For recall that Frege initially suggests that there is parity between all objects (of an all-inclusive domain), that are given to us independently of value-ranges, regarding the legitimacy of identifying them with their unit classes. In other words: When the focus is laid on the semantics of the formal language - and this is obviously Frege's guiding principle in $\S 10$ - the bounded generalization " $\forall \Delta(\dot{\varepsilon}(\varepsilon=$ $\Delta)=\Delta$ )" seems legitimate, and his argument that the mode of presentation of an object must not be regarded as its immutable property does not thwart it. (I shall say more on this in a moment under (iv).) Furthermore, if it is conceded that regarding the stipulation " $\dot{\varepsilon}(\varepsilon=\Delta)=\Delta$ " all objects that are distinct from both the True and the False and which we likewise do not come across in the garb of value-ranges are on a par with the truth-values on logical grounds, then the situation is as follows: There was simply no need for Frege to go one step further in the footnote and point out that the (allegedly) requisite inclusion of objects referred to by value-range names in a possible generalization of " $\varepsilon(\varepsilon=\Delta)=$ $\Delta$ " is unfeasible (because it offends against the contextual stipulation), unless $\Phi(\xi)$ is a concept under which a single object falls, namely $\dot{\alpha} \Phi(\alpha) .{ }^{63}$ In any event, the question of

$$
\begin{gathered}
\hat{x}(x=\hat{y}(y \neq y)) \\
\hat{z}(z=\hat{x}(x=\hat{y}(y \neq y)))
\end{gathered}
$$

and so forth. These are just the Zermelo numbers." I quote here with Heck's permission.

63 Frege attempts to justify the requirement of including in a possible generalization of " $\dot{\varepsilon}(\varepsilon=\Delta)=$ $\Delta$ " objects that are given to us as value-ranges by adducing his old "different guise argument". Cook and Ebert write: "Frege's permutation argument shows that identifying the truth-values with their singletons will not generate logical difficulties of exactly the sort that would arise were he to identify all objects with their singletons more generally" (Cook \& Ebert, 2016, p. 335). I assume that "all objects" is meant to include those objects that are given to us as value-ranges. Cook and Ebert's statement, taken literally, does not rule out that the twin stipulations in $\$ 10$ might generate logical difficulties, but if so, they would be of a different kind than those that according to Frege's argument in the footnote to $\S 10$ would inevitably arise if " $\varepsilon(\varepsilon=\Delta)=\Delta$ " were intended to apply with unbounded generality. Note that Cook and Ebert do not state explicitly that they consider 
whether the stipulation " $\stackrel{\varepsilon}{\varepsilon}(\varepsilon=\Delta)=\Delta$ " can be consistently extended to objects already referred to by value-range names seems to be insignificant if we attempt to remove the massive indeterminacy of the references of value-range terms which Frege would have to face if he were to regard the range of the first-order variables as all-embracing. And clearly, the question of whether, for example, $\dot{\varepsilon}(\dot{\varepsilon}(\varepsilon=(\varepsilon=\varepsilon))=\varepsilon)$ coincides with Julius Caesar qua $\dot{\varepsilon}(\varepsilon=$ Julius Caesar) would pose the same problem as the question of whether $\grave{\varepsilon}(\varepsilon=(\varepsilon=\varepsilon))$ is identical with $\dot{\varepsilon}(\varepsilon=$ Julius Caesar $) .{ }^{64}$ It seems to me that the entire footnote only makes recognizable sense, if we understand it as a tentative reflection about a possible way to resolve the referential indeterminacy of value-range names which Frege would inevitably face even at the end of $\S 12$ if he considered the first-order domain to be all-encompassing.

(iv) Despite appearances, Frege's argument in the long footnote to $\$ 10$ concerning the possibility of generalizing the dual stipulation in $\$ 10$ does not undermine or invalidate his identification of the True and the False with their unit classes. (Here I am indebted to Richard Heck and an anonymous referee.) By invoking the permutation argument, we can say that the twofold stipulation is logically consistent with the contextual stipulation and, hence, legitimate on logical grounds. In my view, platonist concerns are completely blinded out in $\S 10,{ }^{65}$ but they apparently play a certain role in the footnote that is under discussion. It seems that Frege has a very pragmatic attitude in $\S 10$, one that seemingly runs afoul of the strong platonism which he expresses in other places of his work including Grundgesetze. His credo seems to be: what the eye does not see, the heart does not grieve over. More specifically, in $\$ 10$ Frege probably does not care at all about the question of whether there is some fact of the matter about whether, say, the True is a value-range and, if so, which one it is or which function it may belong to. ${ }^{66}$

the dual stipulation in $\$ 10$ to be logically unassailable. However this may be, if (a) we take Frege at his word (as I did) when at the outset of the footnote he declares that the stipulation $" \dot{\varepsilon}(\varepsilon=\Delta)=\Delta "$ is possible for every object $\Delta$ that is not given to us as a value-range, and if (b) we put this possibility into effect, then doing this should not cause any logical difficulty (if it causes a logical difficulty at all) differing in kind from a logical difficulty that might arise from the identification of the truth-values with their unit classes. To reemphasize, following Frege's line of argument in the footnote, a genuine logical difficulty — quite different in kind from the difficulty that is supposed to emerge from the restrained generalization of " $\varepsilon(\varepsilon=\Delta)=\Delta$ "- -arises only if all objects that are given to us in the guise of value-ranges are included in the generalization of " $\dot{\varepsilon}(\varepsilon=\Delta)=\Delta$ "-with the exception of " $\dot{\varepsilon} \Phi(\varepsilon)$ ", if $\Phi(\xi)$ is a concept under which $\dot{\varepsilon} \Phi(\varepsilon)$ alone falls (as was already said above).

64 Note that in the light of the dual stipulation in $\$ 10$ the identification of $\dot{\varepsilon}(\varepsilon=(\varepsilon=\varepsilon))$ with $\grave{\varepsilon}(\dot{\varepsilon}(\varepsilon=(\varepsilon=\varepsilon))=\varepsilon)$ does not clash with the criterion of identity for value-ranges provided by the contextual stipulation and, hence, would be legitimate from a logical point of view. Due to the dual stipulation in $\S 10$, the True is identical with $\dot{\varepsilon}(\varepsilon=(\varepsilon=\varepsilon))$-and of course with $\dot{\varepsilon}(-\varepsilon)$ or with $\dot{\varepsilon}(\varepsilon=\mathfrak{a}-\mathfrak{a}=\mathfrak{a})$-and the corresponding function $\xi=(\xi=\xi)$ is therefore a concept under which $\dot{\varepsilon}(\varepsilon=(\varepsilon=\varepsilon))$-or $\dot{\varepsilon}(-\varepsilon)$ or $\dot{\varepsilon}(\varepsilon=\mathfrak{a}-\mathfrak{a}=\mathfrak{a})$-alone falls.

65 In recent correspondence (4 May 2017), Richard Heck pointed out to me that the very problem that is under discussion in $\$ 10$ makes no sense if it is read against an extreme platonist background.

66 If there were a (metaphysical) fact of the matter that the True is identical with $\grave{\varepsilon}(-\varepsilon)-$ a case which the contextual stipulation does not rule out-then the first of the twin stipulations in $\$ 10$ would amount to identifying the True with itself. From a metaphysical or platonist point of view, it would then be an unerring stipulation. By contrast, if there were a fact of the matter that the True is identical with $\grave{\varepsilon}\left(\varepsilon=\tau^{\mathfrak{a}}-\mathfrak{a}=\mathfrak{a}\right)$ - a case which the contextual stipulation does not exclude either-then the second stipulation in $\$ 10$ would amount to identifying the False with the True. From a platonist point of view, this stipulation would then miss its mark. To be sure, if the first- 
Seen in this way, he is not bound to rule out, prior to the twofold stipulation in $\S 10$, that the True and the False are classes containing more than one object or no object at all.

I would even go a little further. Suppose that Frege had been asked: If from the point of view of your platonist stance you had to answer the question "Is there a fact of the matter in the mind-independent universe of (Fregean) logical objects whether, say, the True is a value-range, and if so, which one?", what would you say? I think that Frege would probably have answered with "yes". In particular, he might have pointed outalthough in different wording - that mind-independent and epistemically inaccessible facts about the truth-values and value-ranges do not undermine his stipulation in $\$ 10$, since its legitimacy - its consistency with the constraints that he had previously imposed on value-ranges and the truth-values-rests entirely on the permutation argument and the identifiability thesis both of which are grounded in broadly logical considerations.

The only constraint imposed on value-ranges up to $\$ 10$ is the contextual stipulation in $\S 3$. From a logical point of view, this is the only relevant thing that we know about value-ranges up to $\S 10$. Regarding the truth-values, I consider the constraints to be their characteristic marks which Frege mentions in Frege (1893), §2, in particular this one: a declarative sentence refers either to the True or the False, and the True and the False are distinct (logical) objects. In his response to the hypothetical question above, Frege might have added that in general it is not metaphysical, but logical constraints that have a formative influence on the semantics of his concept-script. Plainly, the reference of, say, " $\dot{\varepsilon}(-\varepsilon)=\mathfrak{a}-\mathfrak{a}$ " has not been settled by any stipulation prior to the stipulation in $\S 10$. The contextual stipulation neither confirms nor rules out that a given value-range name corefers, say, with a concept-script name of the False, and that is one important point at issue in $\S 10$, although it is not the only one as can be seen from the fact that prior to $\$ 10$ the reference of "—_ $\dot{\varepsilon} \Phi(\varepsilon)$ " and " $\top \grave{\varepsilon} \Phi(\varepsilon)$ " has not been fixed either. So, from the point of view of his platonism Frege would probably have said that " $\varepsilon(-\varepsilon)=\mathfrak{a}-\mathfrak{a}$ " has a determinate truth-value independently of our judgements and assertions. Yet in the same breath, he would probably have insisted that within the framework of his stipulations-and this is obviously the only thing that matters when he deals with the semantics of valuerange names in $\S 10, \S 31$ - whence his nonchalance vis-vis his platonism- " $c(-\varepsilon)=$ $\mathfrak{a}$ - $\mathfrak{a}$ " lacks a (determinate) reference prior to the stipulation that he is going to make in $\$ 10$. Claiming the opposite would be at odds with what Frege says in the first half of $\S 10$. In the light of his general semantic principles, a truth-value name that is said to contain a referentially indeterminate expression-and this applies prior to $\S 10$, for example, to " $\varepsilon(-\varepsilon)$ " as a constituent of " $\varepsilon(-\varepsilon)=\mathfrak{a}-\mathfrak{a}$ " - cannot be considered to have a determinate reference.

In an extensive correspondence on Grundgesetze, §10 that I initiated, Richard Heck comments on the issues that I have just been discussing: ${ }^{67}$

$\S 10$, as I read it, looks forward to $\S 29-\$ 32$, where Frege attempts to show that every 'name' of his formal language has been provided with a unique reference. $\S 10$ is supposed to deal with certain special cases in

order domain in Grundgesetze is taken to be all-encompassing, then Frege could not even rule out, prior to $\S 10$, that $\grave{\varepsilon}(-\varepsilon)$ and $\grave{\varepsilon}\left(\varepsilon=\tau^{\mathfrak{a}}-\mathfrak{a}=\mathfrak{a}\right)$ coincide with two distinct celestial bodies, say, with Jupiter and Mars.

67 I quote with his permission some passages from an e-mail which he had sent to me on 13 March 2017. 
which such a reference has apparently not yet been provided, including particularly identities of the form:

(*) Name of Truth-Value $=$ Value-range Name

Indeed, the argument of $\S 31$ (some of which is anticipated in $\S 10$ ) is basically supposed to show that, in a sense, these are the only cases you have to worry about: If you have dealt properly with cases of the form $(*)$, then you have provided a unique reference for every name (given all the rest that has also been done). This includes cases like

$$
\text { “- } \dot{\varepsilon}(-\varepsilon) ",
$$

which are mentioned in $\S 10$, but also much more complex cases, in which such things appear as parts of conditionals and within quantifiers, and even when they contain variables (which turns out to be much more problematic than Frege thought and is what, in the end, invalidates his argument).

Now, suppose against this background that someone suggests that there must be some fact of the matter about whether the True is a value-range and, if so, which one it is. Just for concreteness, let's suppose that the True is actually $\dot{\varepsilon}(\varepsilon=\varepsilon)$. This fact does not undermine the permutation argument, since what that argument is supposed to show is that what Frege has said to that point about the semantics of his language does not suffice to assign a reference to (*)-type expressions. Of course, if he were to say additionally (and, we are supposing, truly) that the True is $\dot{\varepsilon}(\varepsilon=\varepsilon)$, then things would be different; but he hasn't yet said anything like that.

So one way to approach the problem, I suppose, would be to try to figure out whether the True "really is" a value-range, and if so which one. But that would obviously run into all kinds of problems. Indeed, I take Frege here to be trying to avoid problems of exactly that sort. Frege seems to think that, in some sense, our only access to value-ranges goes via our appreciation that "if one function... and a second function are so constituted that both always have the same value for the same argument, then one may say instead: the value-range of the first function is the same as the value-range of the second" (Gg II 146). As he emphasizes, that can't be a definition, but it nonetheless seems to be all he thinks we really know about value-ranges, in some sense. If so, then there's no hope of our deciding whether the True is "really" a value-range.

Fortunately, there's another option. The permutation argument also shows that Frege is free to say more about the semantics of his languageprecisely because what he has said leaves (*)-type sentences unresolved. And he is free to do so even if the True is "really" a value-range, namely: $\dot{\varepsilon}(\varepsilon=\varepsilon)$. In that case, the permutation Frege defines will just swap the reference of the terms " $\dot{\varepsilon}(\varepsilon=\varepsilon)$ " and " $\dot{\varepsilon}(-\varepsilon)$ ", and so

$$
\text { “乌 }(-\varepsilon)=\forall x(x=x) \text { " }
$$

will refer to the True in the new model. Note that the references of sentences are not changed. So " $\forall x(x=x)$ " still refers to $\dot{\varepsilon}(\varepsilon=\varepsilon)$, 
i.e., what the True 'really' is; it's just that " $\varepsilon(\varepsilon=\varepsilon)$ " no longer refers to $\grave{\varepsilon}(\varepsilon=\varepsilon)$ but instead to $\stackrel{\varepsilon}{\varepsilon}(-\varepsilon)$, so

$$
\grave{\varepsilon}(-\varepsilon)=\forall x(x=x)
$$

is now the False. (This would be clearer if we used two sorts of notation for the new and old terms, but I'm too lazy to do that right now.) From a platonistic viewpoint, we might now want to say that " $\varepsilon(\varepsilon=\varepsilon)$ " does not now refer to the value-range of $\xi=\xi$. But that need not undermine what Frege is doing. Call them "schmalue-ranges" then.

This, it seems to me, is why the problems discussed in the footnote do not undermine the stipulation Frege makes in $\S 10$. Neither the permutation argument nor the stipulation Frege goes on to make presupposes that expressions like

$$
\text { (\#) “ } \stackrel{\varepsilon}{\varepsilon}(-\varepsilon)=\forall x(x=x) "
$$

do not have antecedently have a reference. We can (charitably) interpret Frege to be arguing by dilemma here: Either (\#) doesn't yet have a reference, in which case we are free to declare that both sides denote the same object; or else it does, but then we can modify the references of value-range terms so that both sides denote the same object. Either way, then, we are free to make some sort of stipulation whose effect is to make (\#) true.

Heck agrees with $\mathrm{e}^{68}$ that the terms " $\dot{\varepsilon}(\varepsilon=\varepsilon)$ " and " $\mathfrak{a}-\mathfrak{a}=\mathfrak{a}$ " are not yet available at the stage of $\S 10$. I think that this is of minor importance for his specific line of argument. ${ }^{69}$ So, let us assume, for the sake of argument, that these names are already available in the concept-script. Heck correctly points out that due to the transition from M1 to M2, we must deal with what seem to be "anomalous" or "nonstandard" value-ranges such as $\dot{\varepsilon}(\varepsilon=\varepsilon)$, called by him "schmalue-ranges". We may introduce the term "oblique value-range name" for a name that in $\mathrm{M} 2$ refers to a schmalue-range as follows: An oblique value-range name

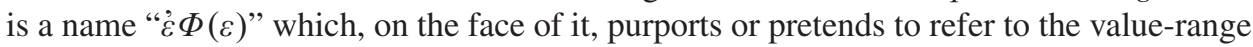
of precisely that function which the function-name " $\Phi(\xi)$ " is supposed to denote, namely $\Phi(\xi)$, but which by virtue of swapping the distinct references of two value-range names (along the lines of Heck's proposal) refers in fact to the value-range of a function $\Psi(\xi)$ which is not coextensive with $\Phi(\xi)$.

In my view, swapping the references of " $\dot{\varepsilon}(\varepsilon=\varepsilon)$ " and " $\varepsilon(-\varepsilon)$ " is not to be seen as a semantic change in isolation, and Heck shares this view. By moving from M1 to M2 we are bound to reinterpret certain other value-range names of the formal language as well. By contrast, the reference of concept-script truth-value names in general is the same in M1 and M2. I assume that by moving from M1 to M2 “_ $\xi$ ” does not undergo any reinterpretation and, as Heck claims, that " $\varepsilon(-\varepsilon)=\mathfrak{a}-\mathfrak{a}=\mathfrak{a}$ " refers to the True in M2. ${ }^{70}$ But how about

68 In his e-mails sent to me on 4 May and 8 October 2017.

69 However, I hasten to add that the unavailability of, say, the term " $\varepsilon\left(\varepsilon=\tau^{\mathfrak{a}}-\mathfrak{a}=\mathfrak{a}\right)$ " in $\S 10$ calls into question the rightfulness of Frege's dual stipulation in $§ 10$; for details see my critical account in $\$ 9.1$.

70 Heck writes in his e-mail of 6 October 2017 (I quote again with his permission): "Yes, swapping the reference of ' $' \varepsilon(\varepsilon=\varepsilon)$ ' and ' $c(-\varepsilon)$ ' will force changes in other terms. This is the point made by Wehmeier and Schroeder-Heister. Really, what we have to do is change not what certain 
the reference of, say, " $\dot{\varepsilon}\left(T^{-\varepsilon}=\varepsilon\right)$ " in M2? It seems to me that it must be $\dot{\varepsilon}(-\varepsilon)$ since in M2 " $\dot{\varepsilon}(\varepsilon=\varepsilon)$ " refers to $\dot{\varepsilon}(-\varepsilon)$ and the reference of " $\mp \xi$ ", like that of " - $\xi$ ", is not changed by passing from M1 to M2. How about the reference of a canonical value-range equation such as " $\dot{\varepsilon}(\varepsilon=\varepsilon)=\grave{\varepsilon}(\varepsilon=(\varepsilon=\varepsilon))$ " or " $\dot{\varepsilon}(\varepsilon=\varepsilon)=\grave{\varepsilon}(\varepsilon=\mathfrak{a}-\mathfrak{a}=\mathfrak{a})$ " in M2? Clearly, in M1 both equations refer to the False. Do they refer to the True in M2? As far as " $\dot{\varepsilon}(\varepsilon=\varepsilon)=\grave{\varepsilon}(\varepsilon=\mathfrak{a}-\mathfrak{a}=\mathfrak{a})$ " is concerned, my answer is "yes". To repeat: " $(\varepsilon=\varepsilon)$ "

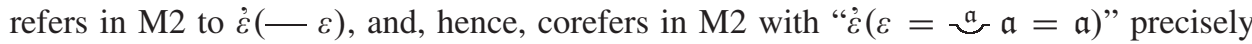
because the reference of " $\mathfrak{a}-\mathfrak{a}=\mathfrak{a}$ " remains unchanged by passing from M1 to M2. On the face of it, it could seem that the answer regarding " $\varepsilon(\varepsilon=\varepsilon)=\grave{\varepsilon}(\varepsilon=(\varepsilon=\varepsilon))$ " is likewise "yes", that is: " $\dot{\varepsilon}(\varepsilon=\varepsilon)$ " seemingly corefers with " $\varepsilon(\varepsilon=(\varepsilon=\varepsilon))$ " in M2. However, in this special case I am less certain since both names " $\stackrel{\varepsilon}{\varepsilon}(\varepsilon=\varepsilon)$ " and " $\dot{\varepsilon}(\varepsilon=(\varepsilon=\varepsilon))$ " contain " $\xi=\xi$ " as a constituent name, although it occurs in them in different syntactic positions. ${ }^{71}$

The upshot is that as far as the semantics of the formal language in M2 is concerned, there are a number of indeterminacies that would have to be resolved. My hunch is that Frege might have felt fairly uneasy if he had thought that for reasons concerning the semantics of the formal language he had to work not only with "ordinary" value-ranges and "standard" value-ranges names but also with "schmalue-ranges" and "oblique" valuerange names in the course of laying the logical foundations of arithmetic. But perhaps he would have thought that the possible advantages to be gained from swapping the references of certain value-range terms by appeal to the permutation argument (if that was considered to be good option to solve a semantic problem) would outweigh the possible disadvantages.

\$9. Closing. We have seen that Frege cannot have his cake and eat it when in Grundgesetze he engages in fixing the references of canonical value-range names up to uniqueness by completing the unfinished business left by the contextual stipulation in $\S 3$. The proposed method in $\S 10$ to accomplish this is hopelessly doomed to failure if the first-order domain

terms refer to but change the reference of the smooth breathing: what function from functions to objects it denotes. And that will induce all sorts of changes. See pp. $107 \mathrm{ff}$. of my book for discussion. But yes, this does not imply any change in the reference of the horizontal. In the model itself, there is still some fixed object that is, e.g., the denotation of $\forall x(x=x)$. That has not changed. This actually plays an important role in the argument I give on p. 109." Heck refers here to Heck (2012).

71 Heck writes in his e-mail of 6 October: "If you think about it in terms of swapping the reference of terms, then it is actually impossible to change the truth-values of identities. Otherwise, the 'permutation' would not be one-one. But if we're changing the reference of the smooth breathing, then we can affect the truth-values of such identities. It may be that I was not very clear about this in the earlier e-mail [Heck refers here to his e-mail of 13 March from which I have quoted several passages]. Whether we get that effect with the particular change I was discussing there, I'm not sure. If so, that would reveal another problem with the argument of §31. But I think it might be possible to argue that, so long as the permutation does not affect which value-ranges are truth-values, it will not affect the truth-values of any identity-statements." - "The goal of $\S 10$, as I see it, is to reduce the indeterminacy to an acceptable level. It is (supposed to be) a consequence what Frege argues in $\$ 29-\$ 32$ that, after the additional stipulations have been made in $\$ 10$, there is no indeterminacy so far as the truth-values of sentences of begriffsschrift are concerned: Every expression of begriffsschrift has (been proven to have) a unique reference, and what that ultimately seems to mean (when you unwind all the specifications in \$29) is, basically, that all the 'names of truth-values' do. So, while indeterminacy does remain, it is irrelevant to what Frege does in the rest of the book. The last paragraph of $\$ 10$ makes a lot of sense read in this light." 
is taken to be all-inclusive. And recall that the all-inclusiveness of the domain is most obviously suggested by Frege's definitions of first-level function-names. What then is the driving force behind the nonexclusiveness thesis that motivates him to defend it so forcefully in several places of his work?

To all appearances, Frege is convinced that the requirement that a (first-level) function, concept or relation must have sharp boundaries can be met only if the range of admissible arguments is regarded as all-embracing. However, he does not mention any compelling reason why he thinks that this condition must be satisfied. His conviction that utmost generality and universal applicability are the salient features of both logic and (cardinal) arithmetic - the domain of the conceptually thinkable and the domain of the countable are considered to be the most comprehensive of all domains ${ }^{72}$ - may have been his principal motive for setting up the thesis that functions, concepts and relations, if they are used in science, must have a value for every appropriate argument (or for every pair of appropriate arguments).

Within classical two-valued logic, Frege's requirement of sharply delimiting concepts and relations may appear intelligible, since for him the requirement is only the law of excluded middle in a different guise. Yet the idea that the requirement, if it is to be sustainable, must go hand in hand with the assumption that the first-order domain includes all the objects there are, is less intelligible. This idea provokes the objection that the requirement could also be met if certain restrictions were imposed on the first-order domain. Prima facie, the domain could be limited to just those logical objects which from Frege's point of view are needed for laying the logical foundations of cardinal arithmetic, real analysis and the arithmetic of complex numbers. Thus, if the first-order domain is said to contain only the True and the False and value-ranges of first-level functions, and if these basic logical objects are or have been completely determined in Frege's sense of "complete determination", then the question of whether or not, for example, Julius Caesar falls under the concept $-\xi$ simply does not arise. However, when reading through the two volumes of Grundgesetze there appear to be grounds for suspicion that Frege would not have been prepared to abandon his nonexclusiveness thesis had he been confronted with the predicament that his strategy in $\S 10-\$ 12$ may provoke.

Be that as it may, let me now try to figure out how far Frege might have got in $\S 10-\$ 12$ regarding his aim of determining completely the reference of " $\dot{\varepsilon} \varphi(\varepsilon)$ ", had he expressly limited the range of the first-order variables to the truth-values and value-ranges, for example most conveniently in $\S 2$, prior to his elucidations of the first primitive function-names of the concept-script. As it turns out, even in this case his plan of conferring a unique reference on each canonical value-range name would come to naught, due to the lacking legitimacy of the identification of the True and the False with special value-ranges. Clearly, this stipulation is the linchpin in Frege's strategy of fixing completely the reference of " $\dot{\varepsilon} \varphi(\varepsilon)$ " via the step-by-step specification of the values of the primitive first-level functions of his system for fitting arguments, and, as we have seen, its key role is later reflected in his attempt to demonstrate that regular value-range names refer to something.

In what follows, I shall advance two arguments against Frege's twin stipulations in $§ 10$. The first argument is set out from the point of view of the syntax of his formal language and discusses the inappropriate but revisable placement of $\S 10$, including the dual stipulation

72 The domain of the measurable, with which Frege was concerned when in Frege (1903) he set out to provide the logical foundations of real analysis, comprises all types of quantity whatsoever. Nonetheless, it is less encompassing than the domain of the countable. 
that he makes at its end. The second argument is based on semantic considerations. If it is granted some force it may call into question, if not undermine the rightfulness of the twin stipulations in $\S 10$. In my view, the argument applies independently of the question of whether the permutation argument and the identifiability thesis that Frege presents in $\S 10$ are formally sound. ${ }^{73}$

\subsection{An argument against the alleged legitimacy of the twin stipulations from the} viewpoint of the syntax of the concept-script. My "syntactic" argument runs as follows: How can Frege believe he had fixed the references of canonical value-range names uniquely at the end of $\S 12$ (which to all appearances he does) even if we assume in his favour, but only for the sake of argument, that he considers the first-order domain of his logical system to contain only the truth-values and value-ranges? At this stage of the exposition of the concept-script, the syntax of the formal language is at best in statu nascendi. In particular, we are lacking reliable criteria to decide whether a given valuerange name such as " $\varepsilon(\varepsilon=\varepsilon)$ " or " $\varepsilon(\varepsilon=, \mathfrak{a}-\mathfrak{a}=\mathfrak{a})$ " is well-formed from the primitive names. Recall that Frege uses the second name when he stipulates-not literally but in substance-that " $\varepsilon(\varepsilon=\mathfrak{a}-\mathfrak{a}=\mathfrak{a})$ " corefers with a name of the False. Yet only if we knew that these value-range names are well-formed according to the formation rules of the concept-script might we be confident that they have been endowed with a unique reference via the stipulations that Frege makes in $\S 3, \S 10-\S 12$. It is true that in $\S 9$ he introduces the value-range notation, but the three rules for the extraction of function-names from more complex names via gap formation are only laid down in $\$ 26$. And the syntactic rule which governs the insertion of a suitable argument expression into the argument-place of a first-, second-, or third-level function-name is officially stated and explained only in $\$ 30$ entitled "Two ways of forming a name", although the criteria of referentiality in $\$ 29$ are essentially formulated in terms of this syntactic operation. Frege seems to take the application of this rule for granted even at an early stage in the exposition of the concept-script, as many examples suggest, but he is not entitled to do so. ${ }^{74}$ At any rate, both names " $\varepsilon(\varepsilon=\varepsilon)$ " and " $\varepsilon\left(\varepsilon=\sim^{\mathfrak{a}} \mathfrak{a}=\mathfrak{a}\right)$ " contain " $\xi=\xi$ " as a constituent name. In $\S 3$, we have seen that the formation of " $\xi=\xi$ " consists in an iterated application of the rule of insertion (= first and second steps of construction) and the subsequent application of the first gap formation rule

73 For a detailed and technically sophisticated analysis of Frege's permutation argument and his identifiability thesis see Wehmeier \& Schroeder-Heister (2005). The authors distinguish between two possible ways of interpreting Frege's line of reasoning in Grundgesetze, §10: a metalogical and a mathematical interpretation. In §6, they argue, by appealing to Schroeder-Heister (1987), that Frege's identifiability thesis, metalogically construed, is false and that consequently the permutation argument under its metalogical interpretation must be invalid. They also argue (p. 53), by drawing attention to the second footnote to Grundgesetze, $\$ 10$, that in all likelihood Frege must have been aware of the counterexamples to the identifiability thesis (metalogically interpreted) that are put forward in Schroeder-Heister (1987). See in this connection the subtle and thorough analysis in Heck (2012), chapter 4 with special emphasis on $\$ 4.5$ 'Are the Arguments in $\$ 10$ Syntactic or Semantic?'. I, for one, tend to sympathize with Heck's view of the matter including his arguments against some of the claims made by Schroeder-Heister and Wehmeier (2005) and hope to be able to comment on them on another occasion.

74 For the sake of explanation, Frege uses " $\xi=\xi$ " and " $\mathfrak{a}-\mathfrak{a}=\mathfrak{a}$ " already in Frege (1893), §8, just after having elucidated the first-order universal quantifier. The elucidation requires him to state more precisely which function $\Phi(\xi)$ is in each case. Frege calls it the corresponding function about which there may be doubts in some cases, which he discusses at length. An innocent reader of the first eight sections of Grundgesetze may wonder how in his syntax Frege gets from " $\xi=\zeta$ " to " $\xi=\xi$ " and " $\mathfrak{a}-\mathfrak{a}=\mathfrak{a}$ ". 
to " $\Delta=\Delta$ ". (" $\Delta$ " is used here as an auxiliary name which represents a well-formed proper name of the concept-script.) And " $\varepsilon(\varepsilon=\varepsilon)$ " results finally from the insertion of " $\xi=\xi$ " into the argument-place of " $\dot{\varepsilon} \varphi(\varepsilon)$ ". Similarly, " $\dot{\varepsilon}(\varepsilon=, \mathfrak{a}-\mathfrak{a}=\mathfrak{a})$ " can only be obtained by first forming " $\xi=\xi$ " and by subsequently applying four times the rule of insertion.

So, it seems that from a syntactic point of view Frege is operating in a vacuum-at least until §26-when he deals with the semantics of nonprimitive concept-script expressions in one way or another. In particular, at the stage of $\S 10$, he is not yet in possession of the linguistic means that are required to effectively make the twin stipulations in $\S 10$ by appeal to the permutation argument and the identifiability thesis. The fact that this stipulation is informal-it is neither a definition nor is it, unlike the contextual stipulation, later encoded as an axiom of the formal theory-means no free ticket for taking for granted the formation rules of the formal language in $\S 10$ as well as in subsequent sections until Frege has reached $\S 26$.

Even if we concede that thanks to the introduction of the value-range notation in $\S 9$ Frege can form " $\&(-\varepsilon)$ " in $\S 10$, he cannot form a name of the True such as "T $\mathfrak{a}-\mathfrak{a}$ " or “ $+\mathfrak{a}-\mathfrak{a}$ " or “ $\mathfrak{a}-\mathfrak{a}=\mathfrak{a}-\mathfrak{a}$ " or “ $\mathfrak{a}-\mathfrak{a}=-\mathfrak{a}-\mathfrak{a}$ ", let alone " $\mathfrak{a}-\mathfrak{a}=\mathfrak{a}$ ", without introducing and legitimizing the syntactic operation of insertion (plus gap formation in the case of “ $\mathfrak{a}-\mathfrak{a}=\mathfrak{a}$ "), nor is he able to form the name of the extension of a concept under which only the False falls such as " $\varepsilon(\varepsilon=\mathfrak{a}-\mathfrak{a})$ " or " $\stackrel{\varepsilon}{\varepsilon}(\varepsilon=\mathfrak{a}-\mathfrak{a})$ ". And as we have seen, due to the unavailability of any gap formation rule in $\S 10$ and, hence, of " $\xi=\xi$ ", his choice of " $\varepsilon(\varepsilon=$ $\left.T^{\mathfrak{a}}-\mathfrak{a}=\mathfrak{a}\right)$ " as a name that is declared to refer to the False is inadmissible, even if insertion were permissible in $\S 10$. Charitably interpreted, at the stage of $\S 12$ Frege could at best claim to have conferred a reference on the name of a value-range of a primitive monadic first-level function, namely only on " $\varepsilon(-\varepsilon)$ ", " $\varepsilon(-\varepsilon)$ " and " $\varepsilon(\backslash \varepsilon)$ "— - result that is miles away from his goal. Value-ranges of dyadic first-level functions (= the double value-ranges) and their names are introduced only in $\$ 36$ where the syntax of the formal language is already in the bag. Admittedly, my "syntactic" argument against Frege's procedure in $§ 10$ $\$ 12$ probably does not reveal a fatal flaw in his methodology. In fact, I think that the flaw could have been remedied if Frege had taken pains to reorganize the exposition of the concept-script. In particular, he could easily have set out the syntax of the formal language at a much earlier stage. Furthermore, in successive sections he could have dealt with the semantics of the concept-script in such a way that the issues raised in $\S 10$ fit in seamlessly and in a terminologically streamlined fashion with the proof of referentiality in $\$ 31$ and the explanatory remarks accompanying it.

In this connection, Richard Heck drew my attention to his observation in Heck (2012), p. 117 that in many ways " $\$ 10$ would have been better placed, say, between $\S 30$ and $\S 31$, since that is its true context. If so, however, then the discussion in $\$ 10$ inherits the semantic character of the discussion in $\$ 31$." I agree with Heck that for methodological and organizational reasons this would have been just the right place for Frege to deploy his arguments of $\S 10$, perhaps along with some illuminating comments (interspersed in the next section, the old §31) on the interplay or "reciprocity" between the piecemeal strategy in $\S 10$ and the demonstration in $\S 31$ that by virtue of the previous stipulations the primitive name " $\varepsilon \varphi(\varepsilon)$ " has indeed been endowed with a unique reference.

In my judgement, even the initial key section for fixing the semantics of value-range terms, namely $\S 3$, is not ideally positioned in the exposition of the concept-script. As far as the elucidations of the primitive function-names " $-\xi$ ", “ $-\xi$ " , $\xi=\zeta$ " and " $\mathfrak{a}-\varphi(\mathfrak{a})$ " are concerned, there is no reference to value-ranges at all (cf. §5-\$8). An explicit appeal to value-ranges was only needed when Frege came to elucidate " $\ \xi$ " in $§ 11$. The values 
of the conditional function for all arguments are specified without explicit reference to value-ranges $(\S 12){ }^{75}$ Thus, I think that Frege should have removed $\S 3$ from its original place and integrated it suitably into what in Grundgesetze is $\$ 9$. More specifically, I think that in order to frame the semantics of his formal language as transparently as possible, Frege should have first made a clear statement concerning the scope of the first-order domain of his logical system (say, in §2), followed by elucidations of the horizontal, negation, identity, the conditional, and the first- and second-order universal quantifiers in partly new $\S 3-\$ 8 .{ }^{76}$ In another section (exactly $\S 9$ ), he could then have introduced the value-range notation and immediately after that the primitive function-name " $\varepsilon \varphi(\varepsilon)$ ", ${ }^{77}$ and hence value-ranges, via the stipulation: "I use the combination of signs ' $\dot{\varepsilon} \Phi(\varepsilon)=\dot{\alpha} \Psi(\alpha)$ ' as coreferential with ' $\mathfrak{a}-\Phi(\mathfrak{a})=\Psi(\mathfrak{a})$ '."78 Furthermore, in a new $\S 10$, Frege could have elucidated the definite description operator " $\ \xi$ ". However, in this case he should either have skipped the opening remarks in $\S 11$ or he should have modified them appropriately without a reference to his tentative, but predictably unsuccessful proposal (in the long footnote to $\S 10)$ to generalize the stipulation " $\varepsilon(\varepsilon=\Delta)=\Delta$ " in such a way that it

75 Frege adds that according to his (standard) elucidation of the function $T_{\zeta}^{\xi}$ in $\$ 12$ and the previous stipulations its values are also fixed for value-ranges as arguments. Yet it does not seem very likely to me that he understands the addition-which results from his proposed piecemeal strategy in $\S 10$ - as a proper part of his prior elucidation of $T_{\zeta}^{\xi}$. One reason for this is that I do not think that in Frege's opinion the elucidation of ${ }_{\zeta \zeta}^{\xi}$ without a reference to value-ranges as arguments leaves

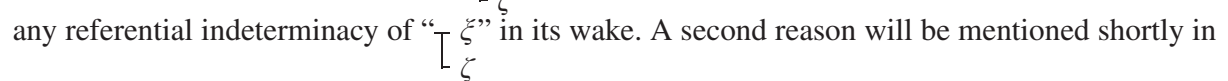
connection with $\xi=\zeta$. Frege writes in $\$ 12$ : "in all other cases the value of the function [that is, of $[\xi]$ shall be the True". What all the other cases (= admissible arguments) specifically are-I assume that he has all objects whatsoever in mind - need not be spelled out from his point of view in order to provide an unobjectionable elucidation of the name of the conditional function and in this way confer a determinate reference on it. Analogous remarks apply to the function-name $" \xi=\zeta$ " which, thanks to its elucidation in $\S 7$, has been provided with a (unique) reference. The subsequent specification (in $\S 10$ ) of the value of $\xi=\zeta$ for the True as $\xi$-argument and a special value-range as $\zeta$-argument (and similarly for the False as $\xi$-argument) is probably not intended as a post ex facto extension or completion of the previous elucidation in $\S 7$. If it were so intended, then Frege would be offending against his rationale that not only piecemeal definitions but also piecemeal elucidations (of primitive names) are inadmissible in his logic. I say this despite the fact that at the end of $\S 10$ he points out that the stipulation of the values of the primitive first-level functions for value-ranges as arguments can be seen as a determination both of the value-ranges and of the relevant function-which I termed "the dual strategy", but also stressed that it is just two sides of the same coin.

76 If Frege had explicitly stated that the first-order variables only range over the truth-values and value ranges, he could have spared himself the trouble of adding the long footnote to $§ 10$. As I have argued, this footnote seems to make sense only if we understand it as a reflection about a possible removal of the referential indeterminacy of value-range names which Frege would still inevitably face even at the end of $\$ 12$, if he considered the first-order domain to be all-embracing. If he did not, why should he bother about the question of whether, say, Caesar and Brutus or Mars and Jupiter, or even objects that are already given to us in the guise of value-ranges, could be identified with their unit classes?

77 As we have seen in $\$ 6$, “' $\varepsilon \varphi(\varepsilon)$ " makes its first separate appearance in Frege (1893), §22.

78 I ignore here the syntactic rule of insertion. Note that in contrast to Frege (1893), $\$ 3$ the contextual stipulation now appears formulated in terms of the value-range notation and the notation for firstorder quantification. 
included also objects that are clad in the garb of value-ranges. And Frege could have added that in order to carry out the proofs of the basic laws of arithmetic in the most effective and most perspicuous manner, he will crucially rely on a function-name-the application operator-which he will later define (in §34) by means of "\६" (which is his first definition in Grundgesetze). By contrast, the name " $\$ ", once it has been employed in the definition of " $\xi \cap \zeta$ ", has almost entirely disappeared in the remainder of Grundgesetze. In fact, it occurs only once again, namely in $\$ 52$, where Frege derives some propositions from Basic Laws V and VI. ${ }^{79}$

In a next step, Frege should have set out the syntax of his formal language, followed by sections on inferences and consequences, the extension of the notation for generality, the criteria of referentiality and the analysis and the suggested removal of the referential indeterminacy of canonical value-range names to which the contextual stipulation gives rise. The latter section would be basically the old $\S 10$, perhaps suitably modified and with reference to the fact that, thanks to the identification of the truth-values with their unit classes, the values of the functions $\backslash \xi$ and $T_{\zeta} \xi$ for value-ranges as arguments are already determined in accordance with their elucidations (in the old $\S 11$ and $\S 12$ ). ${ }^{80}$ Frege could

79 According to Cook (2013, p. A-29), the definition of the application operator is "arguably" the "most important definition" in Grundgesetze. I am not quite sure whether in Frege's judgement this definition is more important for his logicist project than any other definition that he frames in Grundgesetze, but its utmost importance for this project is beyond doubt. It could seem that much depends on the point of view from which one assesses the importance of a Grundgesetze definition in relation to other Grundgesetze definitions. One might, for example, argue that from the point of view of Frege's foundation of cardinal arithmetic, the definition of the cardinality operator in Frege (1893), $\$ 40$ could perhaps be considered to be the most important one. (Recall in this connection Frege's earlier statement in Grundlagen, $\$ 4$ according to which the main task of this book is the definition of cardinal number.) However this may be, here are three arguments of different weight in favour of Cook's claim, although I doubt that one could set up a complete hierarchy for the Grundgesetze definitions in terms of their importance for laying the logical foundations of cardinal arithmetic and real analysis, if that were desirable at all. (1) The definition of the cardinality operator in $\$ 40$ rests inter alia on the use of the application operator. Analogous remarks apply to almost all subsequent definitions, beginning in $\$ 37$ with the definition of the single-valuedness of a relation and ending with the definition of "the circumstance that an object belongs to a series running from an object to an object" in $\$ 158$. The only definitions (after $\S 34)$ in which " $\xi \sim \zeta$ " does not occur are the definitions of " $Q$ " and " 1 " in $\S 41-\S 42$. (2) " $\xi \cap \zeta$ " is designed to create the possibility of representing both monadic and dyadic functions of first level by their value-ranges ("although of course not in such a way that they simply concede their places to them, for that is impossible"), and in this manner to provide a device of using firstlevel functions instead of second-level functions in the system. As I had already indicated in $§ 2$, stepping down from level two to level one regarding the use of functions is most likely intended to bring about logical flexibility, economy and conciseness in the proofs. This argument is of course also mentioned by Cook and it is possibly the strongest. (3) The application operator is by far the most frequently used name of all defined function-names in Grundgesetze, followed by the function-names which Frege defines in Frege (1893), §45, §46, §138, §144. In fact, the application operator is omnipresent in the proofs that he carries out in the two volumes of Grundgesetzein the part called analysis (Zerlegung) as well as in the subsequent purely formal construction (Aufbau) — with the only exception of the simple initial proofs in Frege (1893), §50-\$52 (= the derivation of the principal laws of identity as well as the derivation of some propositions from Basic Laws V and VI).

80 In the Foreword to Grundgesetze, Frege points out (p. XII) that $\$ 10$ should be omitted on a first reading and goes on to observe that the same applies to $\$ 26$ and $\$ 28-\$ 32$. He observes that these sections are not required for our understanding of concept-script sentences. (Thanks 
then have carried out the proof of referentiality, followed by a section-essentially the old $\$ 32$ - in which unfolding and assessing the semantics of the formal language is supposed to come to completion. The remaining sections in his exposition (\$33-§52) would not have required any adjustment or rearrangement.

To end this discussion, let me give an example of what I mean by the desideratum of streamlining the accounts in Frege (1893), $\$ 10$ and in $\S 31$. Generally speaking, I mean the adjustment of Frege's account in $\$ 10$ to his decidedly semantic mode of speaking in $\S 31$, also stressed by Heck in the quotation above. Thus, in a slightly revised section of the old $\S 10$ — ideally placed between $\S 30$ and $\S 31$ - Frege could have suggested that the indeterminacy which he characterizes at the beginning of $\S 10$ can be resolved by determining the reference of any name that results from inserting a name of a truth-value (a truthvalue-name) or a name of a value-range (a value-range-name) into the argument-place(s) of a primitive first-level function-name. Later in this section, he could have continued as follows: The question can only be whether a name of a truth-value refers to a valuerange... Now, the question whether a truth-value name refers to a value-range cannot possibly be decided on the basis of "the stipulation in §3". (Note that this stipulation is already framed in semantic terms just as the similar (possible) stipulation which Frege mentions immediately after having drawn attention to the undecidable question which I have just reformulated in semantic terms.)... Thus, ... it is always possible to determine that an arbitrary (canonical) value-range name refers to the True and that another arbitrary value-range name, which is not coreferential with the first, refers to the False. Let us therefore stipulate that, say, " $\dot{\varepsilon}(-\varepsilon)=\mathfrak{a}-\mathfrak{a}=\mathfrak{a}$ " refers to the True and that " $\dot{\varepsilon}(\varepsilon=$ $\stackrel{\mathfrak{a}}{\mathfrak{a}=\mathfrak{a})=T^{\mathfrak{a}}} \mathfrak{a}=\mathfrak{a}$ " likewise refers to the True. (Names like " $\mathfrak{a}-\mathfrak{a}=\mathfrak{a}$ " or " $\dot{\varepsilon}\left(\varepsilon=\Gamma^{\mathfrak{a}}-\mathfrak{a}=\mathfrak{a}\right)$ " would of course be available if $\S 10$ had been positioned between $\S 30$ and §31.) Accordingly, at the end of the revised section, Frege could have presented his (alleged) achievement as well as the proposed "dual strategy" as follows: We have hereby determined the references of value-range names as far as possible at this stage. Only when we introduce a (primitive) first-level function-name that is not completely reducible to the (first-level) function-names already known will we be able to stipulate what reference it is to have for names of value-ranges as argument-expressions; and this can then be regarded as a determination of the references of value-range names as well as of the reference of that function-name.

9.2. An argument against the alleged legitimacy of the twin stipulations from a semantic point of view. Prior to the presentation of my second argument against the alleged legitimacy of the twin stipulations in $\S 10$, I shall discuss the question of what it means for Frege to "determine logical objects completely". I shall do this chiefly with the aim in mind to set the stage for my second argument. For practical reasons, I shall partly resume Frege's nonsemantic mode of speaking in $\S 10$.

I believe that in Frege's view logical objects could, in principle, be completely determined either (a) by means of an appropriate elucidation of a fundamental primitive second- or higher-order monadic function whose values for admissible arguments are just the desired new objects; (b) by means of an explicit definition of a sortal first-level concept under which the new objects and only those are supposed to fall and which consequently satisfies the principle of completeness, that is, the condition of the sharp delimitation of

to Richard Heck for drawing my attention to Frege's statements.) I, for one, think that for an adequate understanding of some, if not most concept-script sentences, it is at least helpful to know the formation rules of the formal language and the way they interact. 
the concept; (c) by stating criteria of identity for the new objects in terms of a logical abstraction principle and by subsequently making additional stipulations. The latter would be mandatory since Fregean abstraction principles intrinsically involve a massive indeterminacy of reference for term-forming operators, and they do so across the board.

In the case under consideration, Frege most likely regarded (c) as the only viable strategy. For clearly, an elucidation of $\dot{\varepsilon} \varphi(\varepsilon)$ along the lines of the elucidations of the other primitive functions of his system, say, "The value of $\dot{\varepsilon} \varphi(\varepsilon)$ for the function $\Phi(\xi)$ as argument is the value-range of $\Phi(\xi)$ " appears to be ruled out because it illicitly presupposes that we already possess the specific knowledge of value-ranges that is required for using them in the logical construction of arithmetic. ${ }^{81}$ Recall in this connection the fatal assumption that Frege makes prior to his explicit definition of the cardinality operator in Grundlagen, $\S 68$, namely that the reader knows exactly what extensions of concepts are.

Option (b) does not fare better than option (a). At first glance, one might be tempted to assume that Frege could have defined the predicate " $a$ is a value-range" ("VR(a)"), modelled on his definition of " $n$ is a cardinal number" in Frege (1884), §72, and in this way could have achieved his aim of completely determining value-ranges:

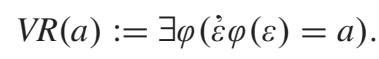

Equipped with this definition, which, let us suppose, satisfies Frege's principle of completeness, he would have been in a position to decide, in principle, for every given object $a$ whether or not it is a value-range. If $a$ is a value-range and is given to us as such, the contextual stipulation or Basic V would tell us whether $a$ is identical with a valuerange $b$ referred to by a canonical value-range name. Unfortunately, the definition rests on the assumption that the value-range operator has already been endowed with a unique reference, which is not the case, at least not before reaching the final stipulation in $\S 12$. Yet if it had been so endowed, then it seems that from Frege's point of view value-ranges already would have been completely determined. This is of course not to say that the definition I tentatively proposed above would be useless, if prior to it the reference of " $\varepsilon \varphi(\varepsilon)$ " had in fact been fixed uniquely.

Let us now examine option (c) which Frege endorses regarding the intended complete determination of value-ranges. As I had underscored earlier, all that we are supposed to know about value-ranges up to the opening of $\S 10$ is that they are governed by or satisfy the criterion of identity embedded in the contextual stipulation. In the exposition of the concept-script, in particular in the layout of its semantics, no "metaphysical" knowledge concerning value-ranges is involved. However, the identification of the True and the False with their unit classes as the outgrowth of the permutation argument provides us with further important knowledge by stipulation about value-ranges and, hence, contributes

81 Recall that in Frege (1893), §9 Frege makes a stipulation concerning " $\check{\varepsilon} \Phi(\varepsilon)$ ”; he does so in addition to his contextual stipulation in $\S 3$. The former bears a certain similarity to the standard elucidations of the other primitive function-names: Generally speaking, " $\delta \Phi(\varepsilon)$ " shall refer to the value-range of the function $\Phi(\xi)$. Suppose, Frege had intended to place the stipulation in $\S 9$ on a par with the elucidations of the other primitive function-names. In this case, the stipulation should have conferred a definite reference on " $\dot{\varepsilon} \varphi(\varepsilon)$ " at once. Consequently, the contextual stipulation in $\S 3$ would be dispensable with respect to fixing the reference of " $\varepsilon \varphi(\varepsilon)$ ". But it is of course not dispensable. Frege crucially refers to it at the outset of $\S 10$, but does not mention the stipulation

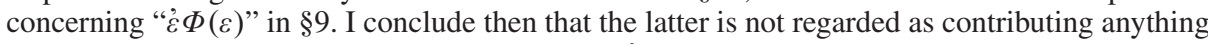
essential to the determination of the reference of " $\dot{\varepsilon} \varphi(\varepsilon)$ " or of the reference of a canonical valuerange name " $£ \Phi(\varepsilon)$ ". For more details concerning the status of the stipulation in $\S 9$ see Schirn (2018b); see also Heck (2012), §5.4, pp. 129ff. 
essentially to their (alleged) complete determination. (i) that every value-range whose correlated function is coextensive neither with $-\xi$ nor with $\xi=\pi \mathfrak{a}-\mathfrak{a}=\mathfrak{a}$, is distinct from both the True and the False; ${ }^{82}$ (ii) that $\grave{\varepsilon}(-\varepsilon)$ or $\dot{\varepsilon}(\varepsilon=(\varepsilon=\varepsilon))$ or $\grave{\varepsilon}(\varepsilon=\mathfrak{a}-\mathfrak{a}=$ $\mathfrak{a})$ - or the extension of any (intensionally speaking) other concept that is coextensive with - $\xi$-is the only value-range that falls under $-\xi$; and correspondingly (iii) that all value-ranges with the sole exception of $\dot{\varepsilon}(-\varepsilon)$ fall under $\top \xi$; (iv) that $\dot{\varepsilon}(-\varepsilon)$ qua the True and $\dot{\varepsilon}(\varepsilon=, \mathfrak{a}-\mathfrak{a}=\mathfrak{a})$ qua the False are the only values of the concepts and relations of the formal system for admissible arguments. ${ }^{83}$ The process of yielding the knowledge by stipulation about value-ranges that Frege considers to be requisite for operating with them in a well-founded way in his logical theory is completed as soon as the values of the definite description function and those of the conditional function for value-ranges as arguments have been specified. ${ }^{84}$

If the first-order domain in Grundgesetze were indeed taken to consist only of the truthvalues and value-ranges, then, on the face of it, the stepwise determination of the values of the primitive first-level functions for the truth-values and value-ranges may seem to be a suitable strategy for achieving the goal of a complete determination of the references of value-range names. In $\S 7$, we have seen that the restriction to primitive functions in this procedure is directly bound up with Frege's central strategic idea in $\S 31$ : in order to prove that all well-formed concept-script names are referential it suffices to show that every primitive function-name of the formal language has a reference, thanks to the inductive assumption that the formation rules, whenever they are applied in order to construct new, nonprimitive names, preserve the property of being referential. ${ }^{85}$ This idea seems basically to be a promising one, and it may render the proposed strategy in $\$ 10$ with an eye to $\$ 29$ $\S 31$ plausible.

So far, so good. However, in what follows, I present a semantic argument against the alleged rightfulness of the twin stipulations in $\S 10$, while bearing in mind Frege's own methodological standards.

82 See in this respect also Frege's letter to Russell of 29.6.1902 (Frege, 1976, p. 219).

83 In saying this, I assume for the sake of argument that even names like " $\grave{\varepsilon}(\varepsilon=(\varepsilon=\varepsilon))$ " and " $\grave{\varepsilon}(\varepsilon=\mathfrak{a}-\mathfrak{a}=\mathfrak{a})$ " are already available at the stage of $\S 10$.

84 Note that at the end of $\$ 10$, in contrast to its beginning, Frege requires that the values of further primitive first-level functions - that he regards as irreducible and indispensable for carrying out his logicist programme-are determined only for value-ranges as arguments. This restriction might be due to the fact that the truth-values had already been identified with special value-ranges. The former now figure as values of $\grave{\varepsilon} \varphi(\varepsilon)$ for certain arguments and, hence, as objects satisfying he contextual stipulation, that is, they are value-ranges. Thus, if contrary to what the phrase "just as for all other arguments" at the beginning of $\S 10$ suggests, Frege regarded the first-order domain as containing only the True and the False and value-ranges, then his choice of phrasing at the end of $\$ 10$ ("for value-ranges as arguments") would appear intelligible.

85 In $\S 10$, this point is passed over in silence or simply taken for granted by Frege, although it stands in need of an argument. Frege writes at the beginning of $\S 10$ : "If we assume that $X(\xi)$ is a function that never receives the same value for different arguments, then exactly the same criterion for recognition holds for the objects whose names have the form ' $X(\dot{\varepsilon} \Phi(\varepsilon))$ ' as for the objects whose signs have the form ' $\grave{\varepsilon} \Phi(\varepsilon)$ '." When using the phrase "the objects whose signs have the form ' $\grave{\varepsilon} \Phi(\varepsilon)$ "' he does not, however, presuppose that names of the form " $\grave{\varepsilon} \Phi(\varepsilon)$ " refer to objects that he has already completely determined. It is clear that at this stage of his line of argument in $\S 10$ Frege must express himself in one way or another in order to get his point across. Making the presupposition just mentioned would be in flagrant contradiction with his subsequent statement that by his stipulation in $\S 3$ the reference of a name such as " $\delta \Phi(\varepsilon)$ " is by no means yet completely determined. But Frege does not commit this error, if we give him his grain of salt. 
In my view, it seems hardly disputable that Frege cannot treat incompletely determined references of value-range names as if they had been completely determined. But this is precisely what he does in $\S 10$ when he comes to identify the primitive objects of logic, the True and the False, with special value-ranges. On the one hand, it is clear from his opening remark in $\S 10$ that the contextual stipulation does not yet by any means fix the reference of a name such as " $\varepsilon \Phi(\varepsilon)$ " completely. And we further know, again from his own statement, that he has still some way to go to achieve a complete removal of the indeterminacy in question even if the domain comprises only the truth-values and valueranges. On the other hand, it seems that in $\S 10$ he takes at least the value-ranges $\dot{\varepsilon}(-\varepsilon)$

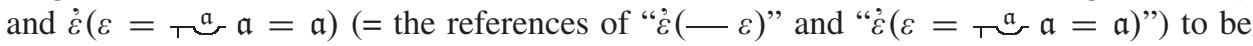
fully determined. If he did not, how could he feel entitled to identify the True with the first value-range (the reference of the first value-range name) and the False with the second (the reference of the second value-range name), even if we assume in his favour that both the permutation argument and the identifiability thesis are formally sound? Moreover, in the light of the identifiability thesis not only $\dot{\varepsilon}(-\varepsilon)$ and $\dot{\varepsilon}\left(\varepsilon=r^{\mathfrak{a}}-\mathfrak{a}=\mathfrak{a}\right)$ but any pair of value-ranges of extensionally nonequivalent monadic first-level functions of the system would have to be regarded as completely determined, contrary to Frege's pronouncements in $\$ 10$. So, at least on the face of it we seem to encounter another incoherence in $\$ 10$ besides the one that I discussed in $§ 9.1$.

Suppose, for the sake of argument, that Frege miraculously succeeded in completely fixing the references of value-range names prior to his identification of the truth-values with $\grave{\varepsilon}(-\varepsilon)$ and $\grave{\varepsilon}\left(\varepsilon=\tau^{\mathfrak{a}}-\mathfrak{a}=\mathfrak{a}\right)$. Plainly, in this case the identification in question would lose its momentum and degenerate into an idle wheel. Yet due to the fundamental role that the primitive function $\xi=\zeta$ plays in the piecemeal process of providing every canonical value-range name with a unique reference, the twofold stipulation in $\S 10$ is considered to be the key element in this process. In Frege's view, the complete determination of the references of value-range names, once it is achieved, seems to imply that we should be able to decide whether the True or the False is distinct from any value-range or whether it coincides with a particular value-range, that is, with the value of $\dot{\varepsilon} \varphi(\varepsilon)$ for a given monadic first-level function as argument. It seems that in the light of Frege's identification of the truth-values with insufficiently or incompletely determined value-ranges, the former, with which we are supposed to be perfectly familiar in our practice of judging and asserting, are now affected by indeterminacy because they are now value-ranges - a potentially disastrous consequence. In summary then, the identification of the reference of a truth-value name with the yet incompletely fixed reference of a value-range name only preserves the initial referential indeterminacy of the latter ( $=$ the indeterminacy arising from the contextual stipulation in \$3) instead of removing it to a large extent, and even worse, turns the former into objects lacking full determinacy, contrary to Frege's firm belief that it is the two truthvalues that stand out as logical objects by their characteristic determinacy once they have been introduced as the references of special function-value names (sentences).

Let me close with some less critical remarks. When in Grundgesetze, §3 Frege proceeds to fix (at least partially) the reference of the value-range operator by means of the contextual stipulation, could he not have shrugged off any platonist and "mode of being given" concerns and stipulated outright that both the True and the False are distinct from every value-range? As we have seen, when by appeal to the permutation argument and the identifiability thesis he decided in $\$ 10$ to identify the True and the False with their unit classes, it seems that for pragmatic reasons he completely disregarded his commitment to platonism-although otherwise this commitment is a common theme throughout his 
logicism ${ }^{86}$ - and his closely related "mode of being given" caveat in the footnote to $\S 10$, which rehearses an earlier argument from Grundlagen. So, could Frege not have adopted a similarly insouciant or relaxed attitude before arriving at $\S 10$, say as early as in $\S 3$ of Grundgesetze, and combined the contextual stipulation with a stipulation stating that the reference of every truth-value name of the formal language-which according to my introduction and use of this term is a name which has the syntactic structure of a declarative sentence, refers either to the True or to the False and expresses a thought-is distinct from the reference of every canonical value-range name? The risk that in the course of laying out the formal system the True and the False might eventually crop up as value-ranges would have been extremely small, if there was any such a risk at all. And in contrast to the twofold stipulation in \$10-whose formal legitimacy Frege bases only and exclusively on the permutation argument as a kind of magic key to solving his semantic problem - the alternative stipulation I considered would not have required a sophisticated argument. In short, if Frege had acquired a taste for such an alternative option and eventually had regarded it as viable without residual scruples, and if he had additionally stipulated that the first-order domain contains only the truth-values and value-ranges, then it seems he might have claimed on plausible grounds that the references of canonical value-range names are fixed uniquely once the syntax of the formal language is finalized. For arguably, the semantic interplay of the elucidations of the primitive first-level function-names, the contextual stipulation plus the stipulation declaring the distinctness of the reference of every truth-value name from the reference of every value-range name might have guaranteed that the reference of every primitive context in which one or two value-range names may occur is fixed or as Frege puts it: that the values of all primitive first-level functions for value-ranges as arguments are determined. According to a statement of Frege's in $§ 10$, no more and no less is required in order to respond appropriately to the problems to which the contextual stipulation despite its merit gives rise. Moreover, showing, as part of the proof of referentiality, that " $\varepsilon \varphi(\varepsilon)$ " has indeed been endowed with a unique reference via the stipulations just mentioned would have been simpler and perhaps also more convincing than the proof that Frege actually gives in $\S 31$ regarding the reference of " $\Leftrightarrow \varphi(\varepsilon)$ ". In sum, pursuing the alternative strategy that I recommended above by appeal to the pronounced pragmatism which Frege displays in the course of dealing with the semantics of value-range names would probably not have resulted in a faux pas had he decided to give preference to it.

The problems with which Frege wrestles when he introduces value-ranges via logical abstraction and attempts to bestow on every canonical value-range name a unique reference lie at the very heart of the philosophy of logic and mathematics that underpins the formal elaboration of the logicist project. Although his exposition in Grundgesetze, $\$ 10$ and §29$\$ 31$ is occasionally affected by lack of clarity and coherence, and although he arguably does not succeed in fixing uniquely the references of value-range names, and despite the fact that his proof of referentiality miscarries, the methodology and formal technique that he applies in these sections, especially his profound insights regarding the referential

86 In Schirn (2014a), §1, I argue that Frege's comments on Basic V in Frege (1903), §146-§147, which culminate in the question "Can our procedure be called a creation?" and his vague response to it, are not in direct conflict with his platonism. I further argue that it is not sufficiently clear what Frege means here exactly with "our procedure". We cannot rule out that he intends to refer here quite generally to the development of the functions and objects dealt with in arithmetic out of the primitive functions of Grundgesetze and not exclusively to the step of logical abstraction in Basic $\mathrm{V}$, namely the transformation of the generality of an equality of function-values into a value-range equality. 
indeterminacy of the terms introduced via logical abstraction as well as his related contextual account of reference, nonetheless deserve to be called pioneering in the history of logic and the foundations of mathematics at the end of the nineteenth century. Together with his earlier groundbreaking work in Grundlagen they gave rise to and paved the way for much fruitful and stimulating work in the current philosophy of mathematics.

\$10. Acknowledgments. I am most grateful to Richard Heck for stimulating and extensive discussion via e-mail of some problems in Grundgesetze, §10. The discussion helped me enormously to improve my article and make it richer in content. Many thanks go to Philip Ebert for his interesting comments on a first draft of §8.2. Special thanks are also due to an anonymous referee for his critical and constructive comments and to the editor of Review of Symbolic Logic, Jamie Tappenden, for his special interest in my article, his encouragement, useful advice, and his final acceptance-with the approval of the managing editor and the referee - of such an unusually long manuscript. I would also like to express my gratitude to the managing editor, Andy Aaarana, for his patience, help and support. I have presented parts of this article in different forms at several universities: Oxford, Stanford, Irvine, Miami, Rio de Janeiro (Brazilian Academy of Philosophy), São Paulo, Federal University of Maranhão (São Luis), University of the Witwatersrand (Johannesburg), Ghent, Helsinki, Stockholm, Cagliari, Florence, Pavia, Pisa, Palermo, Padova, Munich (MCMP), Paderborn, Utrecht, Paris (Panthéon-Sorbonne), Singapore (National University), Tokyo, Hokkaido (Sapporo), Kobe, Kiushu (Fukuoka), Lingnan (Hong Kong), Peking University, Tsinghua (Beijing), Beijing Normal University, Zhejiang (Hangzhou), and Fudan (Shanghai). Many thanks go to the audiences for interesting discussion. I would like to express my deep gratitude (as always) to Dan Mook for carefully reading my essay and for all his valuable suggestions. Many thanks are also due to Kayla A. Riddleberger of Cambridge University Press. Finally, I warmly thank Colin McCullough-Benner for his splendid and meticulous conversion of my word-typescript into $\mathrm{LT}_{\mathrm{E}} \mathrm{X}$ and Marcus Rossberg for his kind support in this respect.

\section{BIBLIOGRAPHY}

Blanchette, P. (2012a). Frege on shared belief and total functions. Journal of Philosophy, 109, 9-39.

Blanchette, P. (2012b). Frege's Conception of Logic. New York: Oxford University Press.

Blanchette, P. (2015). Reply to Cook, Rossberg and Wehmeier. Journal of the History of Analytic Philosophy, 3, 1-13.

Blanchette, P. (2016). Frege on mathematical progress. In Costreie (2016). pp. 3-19.

Cook, R. (2013). How to read Grundgesetze. In Frege, G. Basic Laws of Arithmetic. Derived Using Concept-Script, Vol. I \& II, translated and edited by P. A. Ebert and M. Rossberg, with C. Wright. Oxford: Oxford University Press, pp. A1-A42.

Cook, R. T. (2014). Review of Blanchette (2012b). Philosophia Mathematica, 22, $108-120$.

Cook, R. T. \& Ebert, P. A. (2016). Frege's recipe. The Journal of Philosophy, 113, 309-345.

Costreie, V. S. (editor) (2016). Early Analytic Philosophy. New Perspectives on the Tradition. New York, London: Springer.

Dummett, M. (1973). Frege. Philosophy of Language. London: Duckworth.

Dummett, M. (1981). The Interpretation of Frege's Philosophy. London: Duckworth. 
Dummett, M. (1991). Frege. Philosophy of Mathematics. London: Duckworth.

Frege, G. (1884). Die Grundlagen der Arithmetik. Eine logisch mathematische Untersuchung über den Begriff der Zahl. Breslau: W. Koebner.

Frege, G. (1892). Über Begriff und Gegenstand. Vierteljahrsschrift für wissenschaftliche Philosophie, 16, 192-205. In Frege (1967), pp. 167-178.

Frege, G. (1893). Grundgesetze der Arithmetik. Begriffsschriftlich abgeleitet, Vol. I. Jena: H. Pohle.

Frege, G. (1902). Letter to Russell of 3.8.1902. In Frege (1976), pp. 225-226.

Frege, G. (1903). Grundgesetze der Arithmetik. Begriffsschriftlich abgeleitet, Vol. II. Jena: H. Pohle.

Frege, G. (1967). Kleine Schriften, edited by I. Angelelli. Hildesheim: Georg Olms.

Frege, G. (1976). Wissenschaftlicher Briefwechsel, edited by G. Gabriel, H. Hermes, F. Kambartel, C. Thiel, and A. Veraart. Hamburg: Felix Meiner.

Heck, R. G. (1997). Grundgesetze der Arithmetik I §§29-32. Notre Dame Journal of Formal Logic, 38, 437-474.

Heck, R. G. (1999). Grundgesetze der Arithmetik I §10. Philosophia Mathematica, 7, 258-292.

Heck, R. G. (2005). Julius Caesar and Basic Law V. Dialectica, 59, 161-178.

Heck, R. G. (2011). Frege's Theorem. Oxford: Oxford University Press.

Heck, R. G. (2012). Reading Frege's Grundgesetze. Oxford: Oxford University Press.

Linnebo, Ø. (2004). Frege's proof of referentiality. Notre Dame Journal of Formal Logic, 45, 73-98.

Resnik, M. (1986). Frege's proof of referentiality. In Haaparanta, L. \& Hintikka, J., editors. Frege Synthesized. Essays on the Philosophical and Foundational Work of Gottlob Frege. Dordrecht, Boston: D. Reidel, pp. 177-195.

Ruffino, M. (2002). Logical objects in Frege's Grundgesetze, section 10. In Reck, E., editor. From Frege to Wittgenstein: Perspectives on Early Analytical Philosophy. Oxford: Oxford University Press, pp. 125-148.

Schirn, M. (2003). Fregean abstraction, referential indeterminacy and the logical foundations of arithmetic. Erkenntnis, 59, 203-232.

Schirn, M. (2006a). Concepts, extensions, and Frege's logicist project. Mind, 115, 983-1005.

Schirn, M. (2006b). Hume's principle and axiom V reconsidered: Critical reflections on Frege and his interpreters. Synthese, 148, 171-227.

Schirn, M. (2010). On translating Frege's Die Grundlagen der Arithmetik. History and Philosophy of Logic, 31, 47-72.

Schirn, M. (2013). Frege's approach to the foundations of analysis (1873-1903). History and Philosophy of Logic, 34, 266-292.

Schirn, M. (2014a). Frege on quantities and real numbers in consideration of the theories of Cantor, Russell and others. In Link, G., editor. Formalism and Beyond. On the Nature of Mathematical Discourse. Boston and Berlin: Walter de Gruyter, pp. 25-95.

Schirn, M. (2014b). Frege's logicism and the neo-Fregean project. Axiomathes, 24, 207-243.

Schirn, M. (2016). On the nature, status, and proof of Hume's Principle in Frege's logicist project. In Costreie (2016), pp. 49-96.

Schirn, M. (2018a). Frege on the Foundations of Mathematics, Synthese Library, Studies in Epistemology, Logic, Methodology, and Philosophy of Science. New York, London: Springer. 
Schirn, M. (2018b). Second-order abstraction before and after Russell's paradox. In Ebert, P. and Rossberg, M., editors. Essays on Frege's Basic Laws of Arithmetic. Oxford: Oxford University Press, pp. 433-491.

Schroeder-Heister, P. (1987). A model-theoretic reconstruction of Frege's permutation argument. Notre Dame Journal of Formal Logic, 28, 69-79.

Thiel, C. (1975). Zur Inkonsistenz der Fregeschen Mengenlehre. In Thiel, C., editor. Frege und die Moderne Grundlagenforschung. Meisenheim am Glan: Anton Hain, pp. 134-159.

Wehmeier, K. F. (1999). Consistent fragments of Grundgesetze and the existence of nonlogical objects. Synthese, 121, 309-328.

Wehmeier, K. F. (2015). Critical remarks on Frege's conception of logic by Patricia Blanchette. Journal of the History of Analytic Philosophy, 3, 1-9.

Wehmeier, K. F. \& Schroeder-Heister, P. (2005). Frege's permutation argument revisited. Synthese, 147, 43-61.

MUNICH CENTER FOR MATHEMATICAL PHILOSOPHY UNIVERSITY OF MUNICH

LUDWIGSTRASSE 31

80539 MUNICH, GERMANY

E-mail: matthias.schirn@1rz.uni-muenchen.de 